\title{
Sesquiterpene Lactones from Artemisia argyi: Absolute Configuration and Immunosuppressant Activity
}

Jakob K. Reinhardt, ${ }^{\dagger, \#}$ Amy M. Klemd, ${ }^{\ddagger}, \#$ Ombeline Danton, ${ }^{\dagger}$ Maria De Mieri, ${ }^{\dagger}$ Martin Smieško, ${ }^{\S}$ Roman Huber, ${ }^{\star}$ Thomas Bürgi, ${ }^{\perp}$ Carsten Gründemann, ${ }^{*}$ Matthias Hamburger ${ }^{\dagger}, *$

†Pharmaceutical Biology, Pharmacenter, University of Basel, Klingelbergstrasse 50, 4056 Basel, Switzerland

Center for Complementary Medicine, Institute for Infection Prevention and Hospital Epidemiology, Faculty of Medicine, University of Freiburg, Breisacher Straße 115 B, 79106 Freiburg, Germany

${ }^{\S}$ Department of Molecular Modeling, University of Basel, Klingelbergstrasse 50, 4056 Basel, Switzerland

${ }^{\perp}$ Department of Physical Chemistry, University of Geneva, 30 Quai Ernest Ansermet, 1211 Geneva, Switzerland

\# equally contributed to this work 
Table S1. ${ }^{1} \mathrm{H}$ and ${ }^{13} \mathrm{C}$ NMR Spectroscopic Data (500 $\mathrm{MHz}, \mathrm{CDCl}_{3}$ ) for compounds 1, 2, and 5. . 7 Table S2. ${ }^{1} \mathrm{H}$ and ${ }^{13} \mathrm{C}$ NMR Spectroscopic Data $\left(500 \mathrm{MHz}, \mathrm{CDCl}_{3}\right.$ ) for compounds 3 and 4 ...... 8 Table S3. ${ }^{1} \mathrm{H}$ and ${ }^{13} \mathrm{C}$ NMR Spectroscopic Data $\left(500 \mathrm{MHz}, \mathrm{CDCl}_{3}\right)$ for compounds 6 and $7 \ldots \ldots .9$ Table S4. ${ }^{1} \mathrm{H}$ and ${ }^{13} \mathrm{C}$ NMR Spectroscopic Data (500 MHz, $\mathrm{CDCl}_{3}$ ) for compounds 12 and 16. 10 Table S5. ${ }^{1} \mathrm{H}$ and ${ }^{13} \mathrm{C} \mathrm{NMR}$ Spectroscopic Data $\left(500 \mathrm{MHz}, \mathrm{CDCl}_{3}\right.$ ) for compounds 13 - 15.... 11 Table S6. ${ }^{1} \mathrm{H}$ and ${ }^{13} \mathrm{C}$ NMR Spectroscopic Data $\left(500 \mathrm{MHz}, \mathrm{CDCl}_{3}\right.$ ) for compound 18............ 12

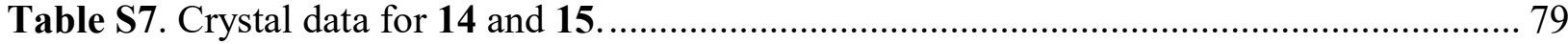

Figure S1. Inhibitory effects of $A$. argyi EtOAc extract on the proliferation of T lymphocytes. Data of three independent experiments were summarized and depicted as means \pm standard deviation in relation to the untreated, stimulated control $(\mathrm{PC} ;=100 \% \pm \mathrm{SD})$. $* \mathrm{P}<0.05, * * \mathrm{P}<$ $0.01, * * * \mathrm{P}<0.001$ 5

Figure S2. Inhibitory effects of compounds 4, 5, 15, and 18 on the proliferation of $\mathrm{T}$ lymphocytes. Data of three independent experiments were summarized and depicted as means \pm standard deviation in relation to the untreated, stimulated control $(\mathrm{PC} ;=100 \% \pm \mathrm{SD}) . * \mathrm{P}<0.05$,

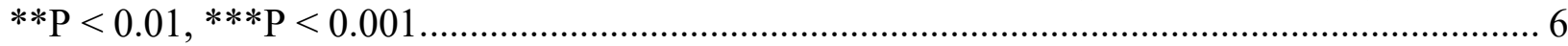

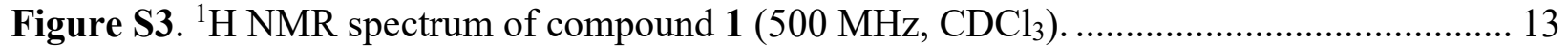

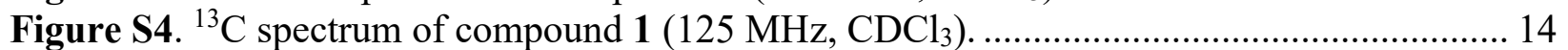

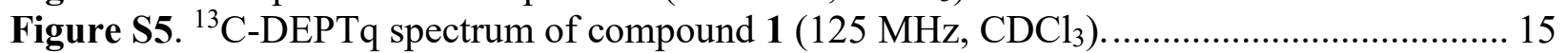

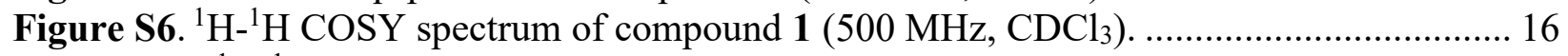

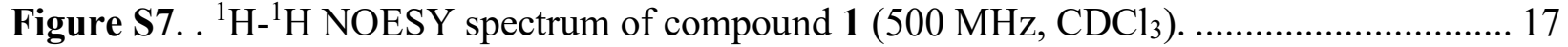

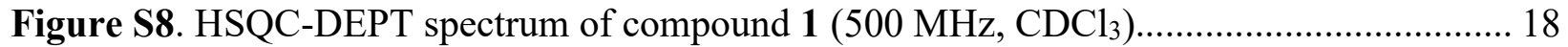

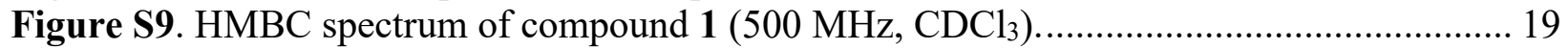

Figure S10. Comparison of experimental and computed IR spectra in chloroform for compound 1 in the region 1900-1050 cm $\mathrm{cm}^{-1}$. Computed spectra (compounds 1, 2 and 5) were scaled according to the overall maximal SimVCD value (0.9865) .......................................... 20

Figure S11. ${ }^{1} \mathrm{H}$ NMR spectrum of compound $2\left(500 \mathrm{MHz}, \mathrm{CDCl}_{3}\right)$.................................. 21

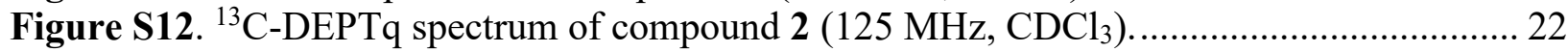

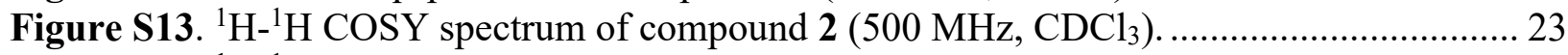

Figure S14. ${ }^{1} \mathrm{H}-{ }^{1} \mathrm{H}$ NOESY spectrum of compound $2\left(500 \mathrm{MHz}, \mathrm{CDCl}_{3}\right)$.......................... 24

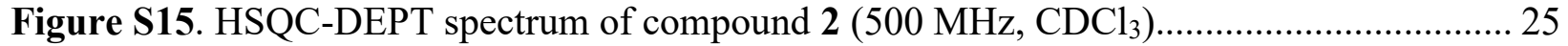

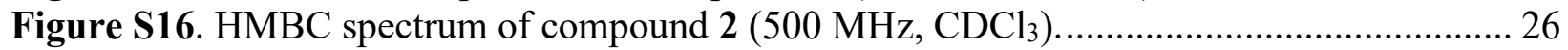

Figure S17. Comparison of experimental and computed ECD spectra for compound $\mathbf{2}$ in $\mathrm{MeOH}$.

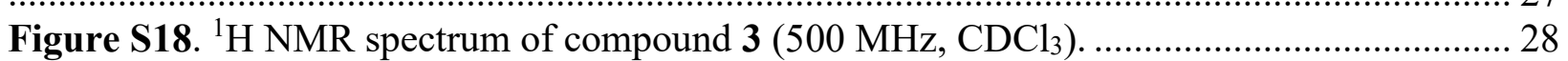

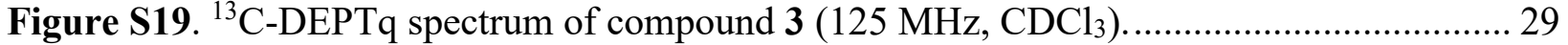

Figure S20. 1H 1H COSY spectrum of compound 3 (500 MHz, CDCl3). ............................ 30

Figure S21. 1H 1H NOESY spectrum of compound $3(500 \mathrm{MHz}, \mathrm{CDC13})$........................... 31

Figure S22. HSQC-DEPT spectrum of compound $3(500 \mathrm{MHz}, \mathrm{CDCl} 3)$............................. 32

Figure S23. HMBC spectrum of compound 3 (500 MHz, CDCl3) .................................. 33

Figure S24. ${ }^{1} \mathrm{H}$ NMR spectrum of compound $4\left(500 \mathrm{MHz}, \mathrm{CDCl}_{3}\right)$. ................................... 34

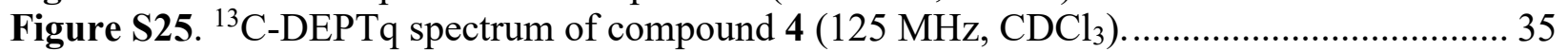

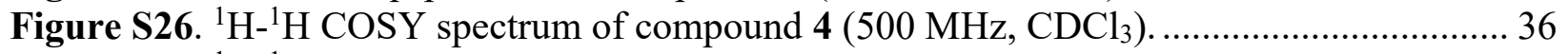

Figure S27. ${ }^{1} \mathrm{H}-{ }^{1} \mathrm{H}$ NOESY spectrum of compound $4\left(500 \mathrm{MHz}, \mathrm{CDCl}_{3}\right)$. .......................... 37

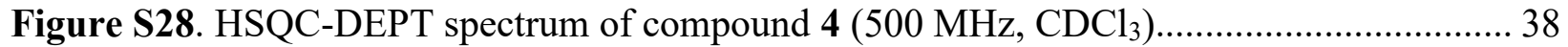

Figure S29. HMBC spectrum of compound 4 (500 $\left.\mathrm{MHz}, \mathrm{CDCl}_{3}\right)$....................................... 39 
Figure S30. Comparison of experimental and computed IR spectra in chloroform for compounds 3 and 4 in the region 1900-1050 $\mathrm{cm}^{-1}$. Computed spectra (compounds 3 and 4 ) were

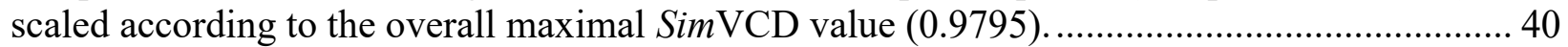

Figure S31. Comparison of experimental and computed VCD spectra in chloroform for compound 4. The region of 1900-1050 $\mathrm{cm}^{-1}$ is shown (A). Similarities (SimVA and SimVCD) of the experimental VA and VCD spectra of 4 to the calculated spectra of possible stereoisomers were plotted as functions of wavenumber scale factor (B). The wavenumber scale factor corresponding to the maximal SimVCD value in B (0.9795) was used to scale the computed

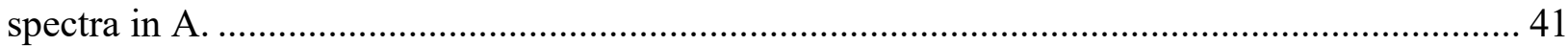

Figure S32. ${ }^{1} \mathrm{H}$ NMR spectrum of compound 5 (500 MHz, DMSO). .................................. 42

Figure S33. ${ }^{13} \mathrm{C}$-DEPTq spectrum of compound 5 (125 MHz, DMSO). ............................... 43

Figure S34. ${ }^{1} \mathrm{H}-{ }^{1} \mathrm{H}$ COSY spectrum of compound 5 (500 MHz, DMSO)............................. 44

Figure S35. ${ }^{1} \mathrm{H}-{ }^{1} \mathrm{H}$ NOESY spectrum of compound 5 (500 MHz, DMSO).......................... 45

Figure S36. HSQC-DEPT spectrum of compound 5 (500 MHz, DMSO) .............................. 46

Figure S37. HMBC spectrum of compound 5 (500 MHz, DMSO) ....................................... 47

Figure S38. Comparison of experimental and computed ECD spectra for compound $\mathbf{5}$ in $\mathrm{MeOH}$. 48

Figure S39. Comparison of experimental and computed IR spectra in DMSO-d 6 for compound 5 in the region 1900-1050 $\mathrm{cm}^{-1}$. Computed spectra (compounds 1, 2 and 5) were scaled according to the overall maximal Sim VCD value (0.9955).

Figure S40. Comparison of experimental and computed VCD spectra in DMSO-d 6 for compound 5. The region of 1900-1050 $\mathrm{cm}^{-1}$ is shown (A). Similarities (SimVA and SimVCD) of the experimental VA and VCD spectra of 5 to the calculated spectra of possible stereoisomers were plotted as functions of wavenumber scale factor (B). The wavenumber scale factor corresponding to the maximal Sim VCD value in B (0.9955) was used to scale the computed

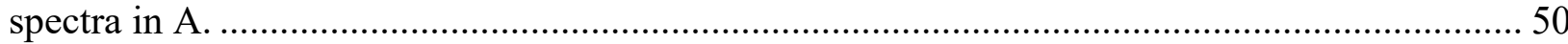

Figure S41. Comparison of experimental and computed UV spectra for compound $\mathbf{6}$ in $\mathrm{MeOH}$.

Figure S42. Comparison of experimental and computed ECD spectra for compound 6 in $\mathrm{MeOH}$.

Figure S43. Comparison of experimental and computed ECD spectra for compound 7 in $\mathrm{MeOH}$.

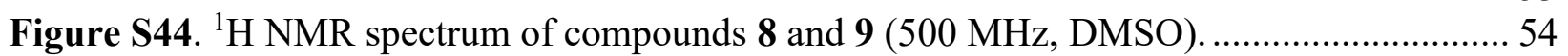

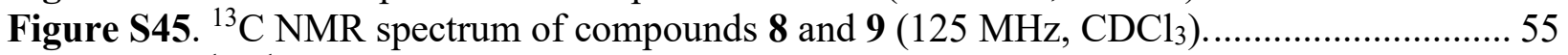

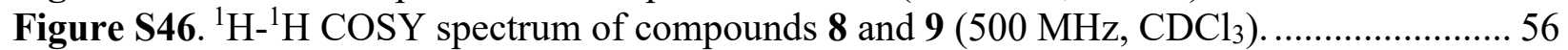

Figure S47. . ${ }^{1} \mathrm{H}-{ }^{1} \mathrm{H}$ NOESY spectrum of compounds 8 and $9\left(500 \mathrm{MHz}, \mathrm{CDCl}_{3}\right) \ldots \ldots \ldots \ldots \ldots . . . . . .57$

Figure S48. HSQC-DEPT spectrum of compounds 8 and $9\left(500 \mathrm{MHz}, \mathrm{CDCl}_{3}\right)$.................. 58

Figure S49. HMBC spectrum of compounds 8 and $9\left(500 \mathrm{MHz}, \mathrm{CDCl}_{3}\right)$........................... 59

Figure S50. Comparison of experimental and computed UV spectra for compound 11 in $\mathrm{MeOH}$.

Figure S51. Comparison of the experimental ECD spectrum for the mixture of compounds 8 and

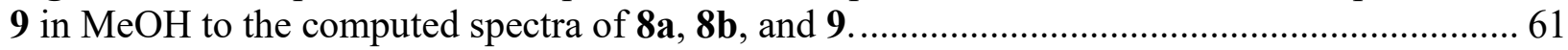

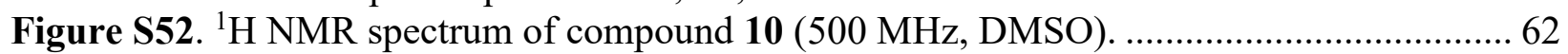

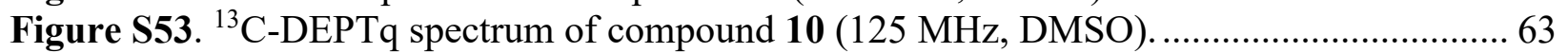

Figure S54. ${ }^{1} \mathrm{H}-{ }^{1} \mathrm{H}$ COSY spectrum of compound 10 (500 MHz, DMSO)............................. 64

Figure S55. ${ }^{1} \mathrm{H}-{ }^{1} \mathrm{H}$ NOESY spectrum of compound 10 (500 MHz, DMSO).......................... 65 
Figure S56 HSQC-DEPT spectrum of compound 10 (500 MHz, DMSO)............................ 66

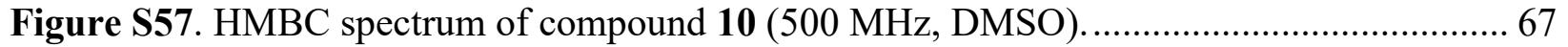

Figure S58. Comparison of the experimental ECD spectrum for compound $\mathbf{1 0}$ in $\mathrm{MeOH}$ to the

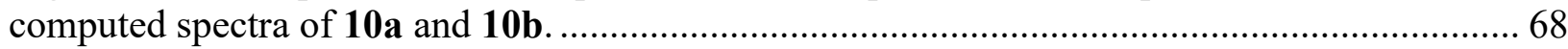

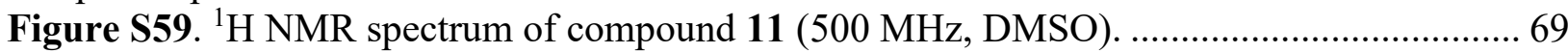

Figure S60. ${ }^{13} \mathrm{C}$-DEPTq spectrum of compound 11 (125 MHz, DMSO) ............................. 70

Figure S61. ${ }^{1} \mathrm{H}-{ }^{1} \mathrm{H}$ COSY spectrum of compound 11 (500 MHz, DMSO)............................ 71

Figure S62. ${ }^{1} \mathrm{H}-{ }^{1} \mathrm{H}$ NOESY spectrum of compound 11 (500 MHz, DMSO).......................... 72

Figure S63. HSQC-DEPT spectrum of compound 11 (500 MHz, DMSO)........................... 73

Figure S64. HMBC spectrum of compound 11 (500 MHz, DMSO).................................. 74

Figure S65. Comparison of experimental and computed UV spectra for compound 11 in $\mathrm{MeOH}$.

Figure S66. Comparison of experimental and computed ECD spectra for compound 11 in

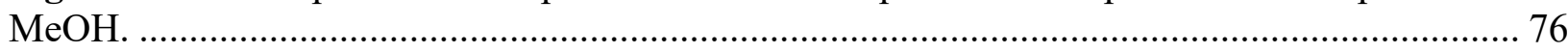

Figure S67. Comparison of experimental ECD spectrum of the mixture of compounds 13 and 14 in $\mathrm{MeOH}(270 \mu \mathrm{g} / \mathrm{mL})$ to the computed spectra of 13a, 13b, and 14. Combined curves were mixed in the ratio of $1: 1$ as determined between compounds 13 and 14 via ${ }^{1} \mathrm{H}$ NMR.............. 77

Figure S68. ORTEP diagram of compound 14 from the X-ray diffraction experiment............ 78

Figure S69. Comparison of experimental and computed ECD spectra for compound 15 in

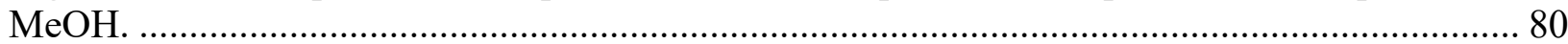

Figure S70. ORTEP diagram of compound 15 from the X-ray diffraction experiment............ 81

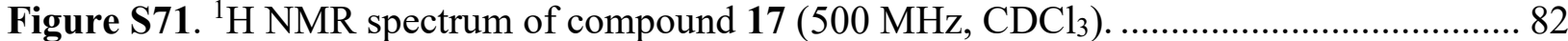

Figure S72. ${ }^{13} \mathrm{C}$-DEPTq spectrum of compound $17\left(125 \mathrm{MHz}, \mathrm{CDCl}_{3}\right)$.............................. 83

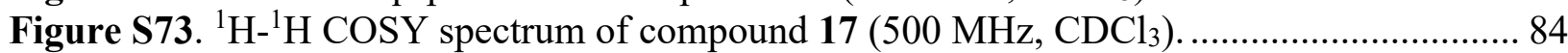

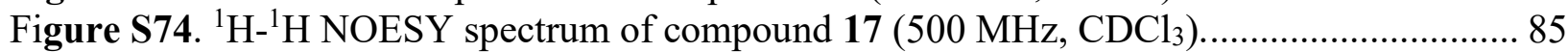

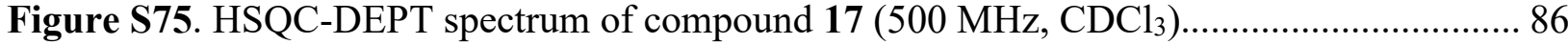

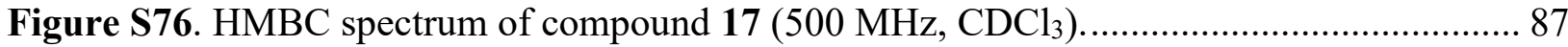

Figure S77. Comparison of experimental and computed UV spectra for compound $\mathbf{1 7}$ in $\mathrm{MeOH}$.

Figure S78. Comparison of experimental and computed ECD spectra for compound 17 in

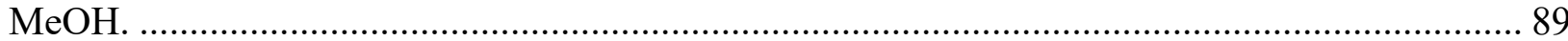

Figure S79. Comparison of experimental and computed ECD spectra for compound 18 in

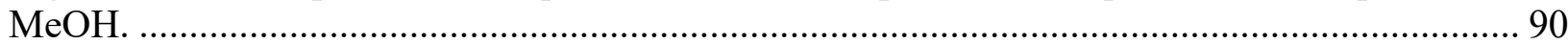

Figure S80. Comparison of experimental and computed IR spectra in chloroform for compound 18 in the region $19001050 \mathrm{~cm}^{-1}$. Computed spectrum of $\mathbf{1 8}$ was scaled according to the maximal SimVCD value $(0.9845)$.

Figure S81. Comparison of experimental and computed VCD spectra in chloroform for compound 18. The region of $1900-1050 \mathrm{~cm}^{-1}$ is shown (A). Similarities (SimVA and SimVCD) of the experimental VA and VCD spectra of $\mathbf{1 8}$ to the calculated spectra of possible stereoisomers were plotted as functions of wavenumber scale factor (B). The wavenumber scale factor corresponding to the maximal SimVCD value in B (0.9845) was used to scale the computed spectra in A. 


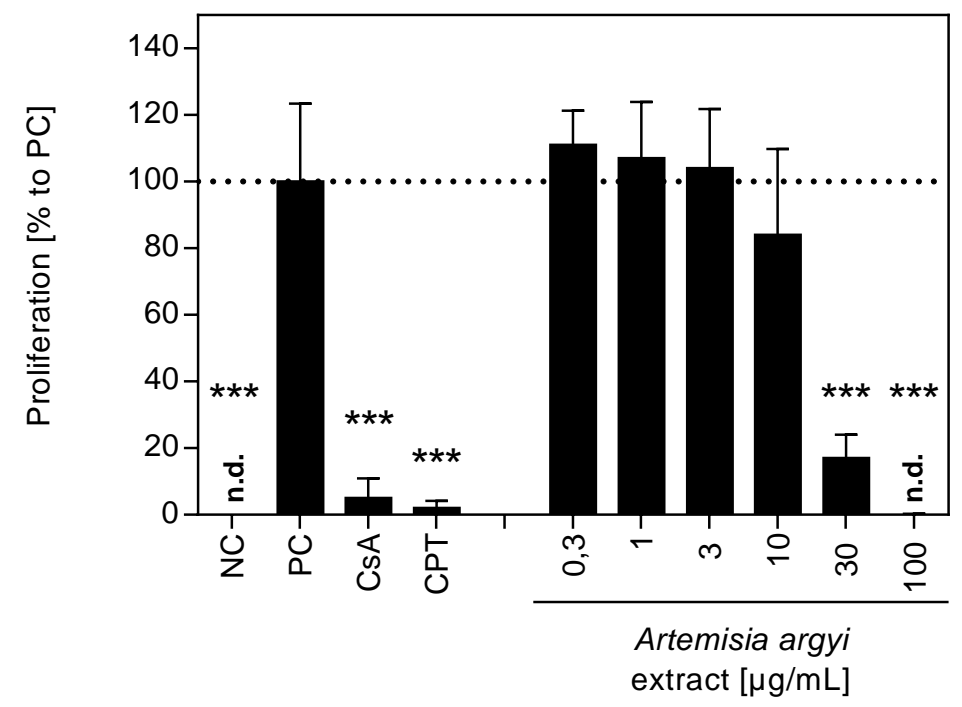

Figure S1. Inhibitory effects of $A$. argyi EtOAc extract on the proliferation of $\mathrm{T}$ lymphocytes. Data of three independent experiments were summarized and depicted as means \pm standard deviation in relation to the untreated, stimulated control $(\mathrm{PC} ;=100 \% \pm \mathrm{SD}) . * \mathrm{P}<0.05, * * \mathrm{P}<$ $0.01,{ }^{* * *} \mathrm{P}<0.001$. 

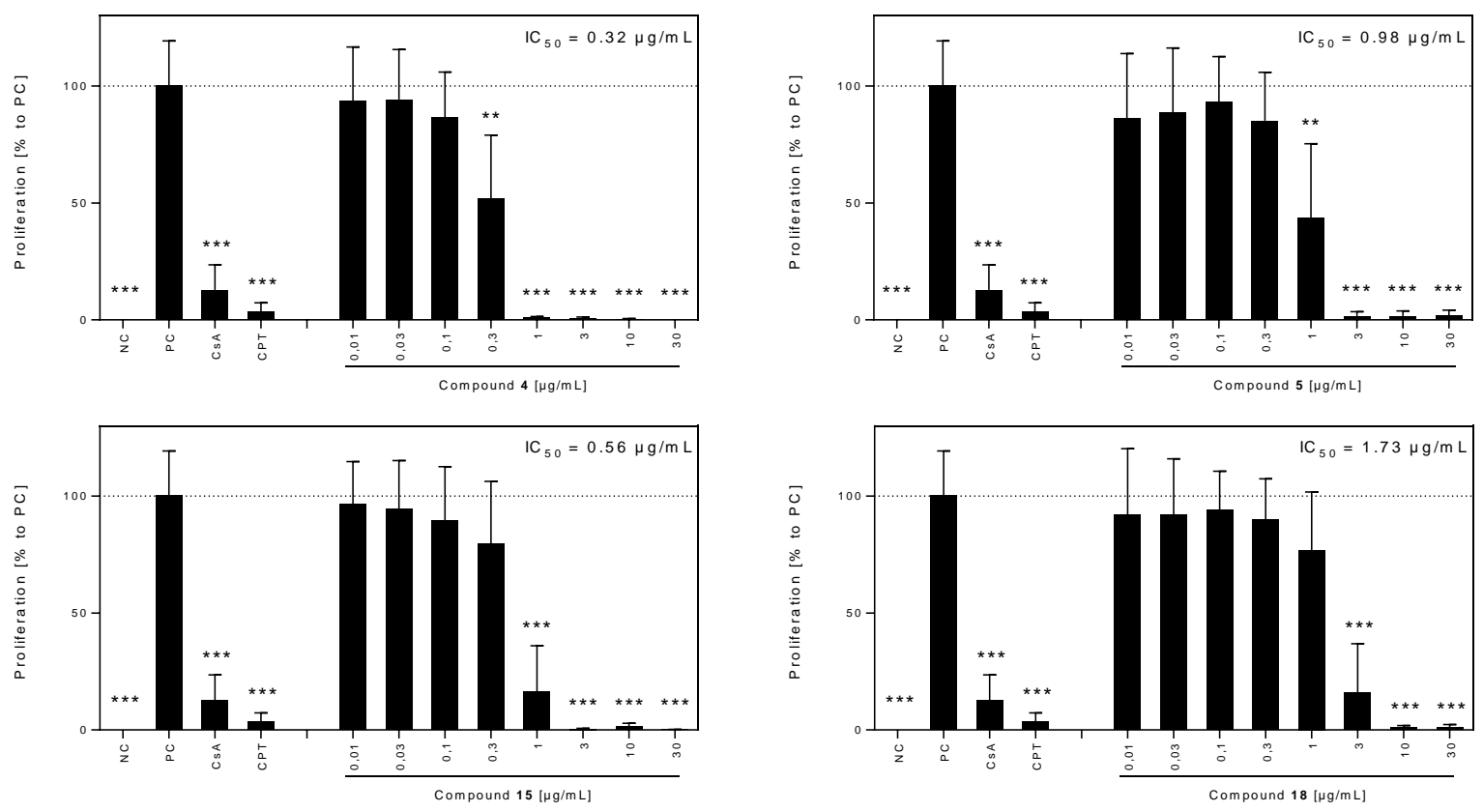

Figure S2. Inhibitory effects of compounds 4, 5, 15, and 18 on the proliferation of $\mathrm{T}$ lymphocytes. Data of three independent experiments were summarized and depicted as means \pm standard deviation in relation to the untreated, stimulated control $(\mathrm{PC} ;=100 \% \pm \mathrm{SD}) .{ }^{*} \mathrm{P}<0.05$, $* * \mathrm{P}<0.01, * * * \mathrm{P}<0.001$. 
Table S1. ${ }^{1} \mathrm{H}$ and ${ }^{13} \mathrm{C}$ NMR Spectroscopic Data (500 MHz, $\mathrm{CDCl}_{3}$ ) for compounds $\mathbf{1}, \mathbf{2}$, and $\mathbf{5}$.

\begin{tabular}{|c|c|c|c|c|c|c|}
\hline \multirow[b]{2}{*}{ position } & \multicolumn{2}{|r|}{1} & \multicolumn{2}{|r|}{$2^{c}$} & \multicolumn{2}{|r|}{$5^{b}$} \\
\hline & $\delta_{\mathrm{C}}$, type & $\delta_{\mathrm{H}}(J$ in $\mathrm{Hz})$ & $\delta_{\mathrm{C}}$, type & $\delta_{\mathrm{H}}(J$ in $\mathrm{Hz})$ & $\delta \mathrm{c}$, type & $\delta_{\mathrm{H}}(J$ in $\mathrm{Hz})$ \\
\hline 1 & $79.8, \mathrm{C}$ & & $78.2, \mathrm{C}$ & & 77.8, C & \\
\hline 2 & $58.7, \mathrm{CH}$ & $3.47, \mathrm{~d}(0.9)$ & $55.4, \mathrm{CH}$ & $3.54, \mathrm{~d}(0.9)$ & $56.0, \mathrm{CH}$ & 3.63 , br. s \\
\hline 3 & $58.3, \mathrm{CH}$ & $3.28, \mathrm{~d}(0.9)$ & $57.6, \mathrm{CH}$ & $3.35, \mathrm{~d}(0.9)$ & $57.0, \mathrm{CH}$ & 3.40 , br. $\mathrm{S}$ \\
\hline 4 & 73.7, C & & $71.8, \mathrm{C}$ & & $70.2, \mathrm{C}$ & \\
\hline 5 & $50.3, \mathrm{CH}$ & $2.55, \mathrm{~d}(11.9)$ & $44.7, \mathrm{CH}$ & $2.26, \mathrm{~d}(10.7)$ & $42.5, \mathrm{CH}$ & $2.81, \mathrm{~d}(11.0)$ \\
\hline 6 & $78.3, \mathrm{CH}$ & $4.22, \mathrm{dd}(11.9,9.5)$ & $81.1, \mathrm{CH}$ & $4.12, \operatorname{dd}(10.7,10.7)$ & $82.4, \mathrm{CH}$ & $3.91, \mathrm{dd}(11.0,10.4)$ \\
\hline 7 & 45.2a, $\mathrm{CH}$ & $3.39, \mathrm{~m}$ & $51.3, \mathrm{CH}$ & $2.57, \mathrm{~m}$ & $44.0, \mathrm{CH}$ & $3.25, \mathrm{~m}$ \\
\hline \multirow[t]{2}{*}{8} & $23.7, \mathrm{CH}_{2}$ & $2.35, \mathrm{~m}$ & $20.2, \mathrm{CH}_{2}$ & $1.98, \mathrm{~m}$ & $22.2, \mathrm{CH}_{2}$ & $2.10, \mathrm{~m}$ \\
\hline & & $1.52, \mathrm{~m}^{a}$ & & $1.62, \mathrm{~m}^{a}$ & & $1.45, \mathrm{~m}^{a}$ \\
\hline \multirow[t]{2}{*}{9} & $34.3, \mathrm{CH}_{2}$ & 2.01, ddd $(15.0,6.1,4.3)$ & $42.2, \mathrm{CH}_{2}$ & 2.17, ddd $(15.6,6.4,2.8)$ & $37.0, \mathrm{CH}_{2}$ & $1.68, \mathrm{~m}$ \\
\hline & & 1.83, ddd $(15.0,10.7,6.1)$ & & 1.80, ddd $(15.0,11.4,3.2)$ & & \\
\hline 10 & $72.4, \mathrm{C}$ & & $69.4, \mathrm{C}$ & & $70.4, \mathrm{C}$ & \\
\hline 11 & $139.6, \mathrm{C}$ & & $138.8, \mathrm{C}$ & & 139.6, C & \\
\hline 12 & $169.4, \mathrm{C}$ & & 169.2, C & & $169.8, \mathrm{C}$ & \\
\hline \multirow[t]{2}{*}{13} & $120.2, \mathrm{CH}_{2}$ & $6.21, \mathrm{~d}(3.4)$ & $118.3, \mathrm{CH}_{2}$ & $6.16, \mathrm{~d}(3.1)$ & $118.8, \mathrm{CH}_{2}$ & $6.02, \mathrm{~d}(3.7)$ \\
\hline & & $5.50, \mathrm{~d}(3.1)$ & & $5.44, \mathrm{~d}(3.1)$ & & $5.57, \mathrm{~d}(3.4)$ \\
\hline 14 & $19.4, \mathrm{CH}_{3}$ & $1.57, \mathrm{~s}^{a}$ & $19.6, \mathrm{CH}_{3}$ & $1.57, \mathrm{~s}^{a}$ & $19.6, \mathrm{CH}_{3}$ & $1.41, \mathrm{~s}^{a}$ \\
\hline 15 & $27.2, \mathrm{CH}_{3}$ & $1.16, \mathrm{~s}$ & $27.6, \mathrm{CH}_{3}$ & $1.41, \mathrm{~s}$ & $26.9, \mathrm{CH}_{3}$ & $0.94, \mathrm{~s}$ \\
\hline
\end{tabular}


Table S2. ${ }^{1} \mathrm{H}$ and ${ }^{13} \mathrm{C}$ NMR Spectroscopic Data $\left(500 \mathrm{MHz}, \mathrm{CDCl}_{3}\right)$ for compounds 3 and 4.

\begin{tabular}{|c|c|c|c|c|}
\hline \multirow[b]{2}{*}{ position } & \multicolumn{2}{|r|}{3} & \multicolumn{2}{|r|}{4} \\
\hline & $\delta_{\mathrm{C}}$, type & $\delta_{\mathrm{H}}(J$ in $\mathrm{Hz})$ & $\delta_{\mathrm{C}}$, type & $\delta_{\mathrm{H}}(J$ in $\mathrm{Hz})$ \\
\hline 1 & $204.8, \mathrm{C}$ & & $204.9, \mathrm{C}$ & \\
\hline 2 & $132.9, \mathrm{CH}$ & $5.94, \mathrm{~d}(5.8)$ & $133.4, \mathrm{CH}$ & $6.01, \mathrm{~d}(5.8)$ \\
\hline 3 & $165.9, \mathrm{CH}$ & $7.33, \mathrm{~d}(5.8)$ & $167.2, \mathrm{CH}$ & $7.44, \mathrm{~d}(5.8)$ \\
\hline 4 & 77.9, C & & 77.3, C & \\
\hline 5 & $58.1, \mathrm{CH}$ & $2.23, \mathrm{~d}(7.3)$ & $56.6, \mathrm{CH}$ & $2.50, \mathrm{~m}^{a}$ \\
\hline 6 & $80.6, \mathrm{CH}$ & $4.45, \mathrm{dd}(7.3,3.1)$ & 79.7, $\mathrm{CH}$ & $4.52, \mathrm{dd}(5.8,2.1)$ \\
\hline 7 & $40.7, \mathrm{CH}$ & $3.27, \mathrm{~m}$ & $39.7, \mathrm{CH}$ & $3.50, \mathrm{~m}$ \\
\hline 8 & $28.2, \mathrm{CH}_{2}$ & $1.70, \mathrm{~m}$ & 26.1, $\mathrm{CH}_{2}$ & $1.84, \mathrm{~m}$ \\
\hline 9 & $39.4, \mathrm{CH}_{2}$ & $2.39, \mathrm{~m}$ & $39.4, \mathrm{CH}_{2}$ & $2.49, \mathrm{~m}^{a}$ \\
\hline 10 & $207.8, \mathrm{C}$ & & 207.3, C & \\
\hline 11 & $137.8, \mathrm{C}$ & & $137.8, \mathrm{C}$ & \\
\hline 12 & 169.6, C & & $169.2, \mathrm{C}$ & \\
\hline \multirow[t]{2}{*}{13} & $124.1, \mathrm{CH}_{2}$ & $6.06, \mathrm{~d}(2.1)$ & $122.7, \mathrm{CH}_{2}$ & $6.15, \mathrm{~d}(2.7)$ \\
\hline & & $5.51, \mathrm{~d}(1.8)$ & & $5.54, \mathrm{~d}(2.4)$ \\
\hline 14 & $30.0, \mathrm{CH}_{3}$ & $1.41, \mathrm{~s}$ & $30.0, \mathrm{CH}_{3}$ & $1.45, \mathrm{~s}$ \\
\hline 15 & $28.7, \mathrm{CH}_{3}$ & $1.99, \mathrm{~s}$ & $27.2, \mathrm{CH}_{3}$ & $2.08, \mathrm{~s}$ \\
\hline \multicolumn{5}{|c|}{${ }^{a}$ Overlapping signals } \\
\hline
\end{tabular}


Table S3. ${ }^{1} \mathrm{H}$ and ${ }^{13} \mathrm{C}$ NMR Spectroscopic Data $\left(500 \mathrm{MHz}, \mathrm{CDCl}_{3}\right)$ for compounds 6 and 7.

\begin{tabular}{|c|c|c|c|c|}
\hline \multirow[b]{2}{*}{ position } & \multicolumn{2}{|r|}{6} & \multicolumn{2}{|r|}{7} \\
\hline & $\delta_{\mathrm{C}}$, type & $\delta_{\mathrm{H}}(J$ in $\mathrm{Hz})$ & $\delta_{\mathrm{C}}$, type & $\delta_{\mathrm{H}}(J$ in $\mathrm{Hz})$ \\
\hline 1 & $74.9, \mathrm{CH}$ & $3.41, \mathrm{dd}(11.8,4.4)^{a}$ & $75.2, \mathrm{CH}$ & $3.67, \mathrm{dd}(10.1,6.7)^{a}$ \\
\hline $1^{\prime}$ & $170.7, \mathrm{C}$ & & & \\
\hline $1 "$ & $169.7, \mathrm{C}$ & & & \\
\hline \multirow[t]{2}{*}{2} & $36.8, \mathrm{CH}_{2}$ & $1.54, \mathrm{q}(11.9)$ & $32.8, \mathrm{CH}_{2}$ & $2.39, \mathrm{~m}^{a}$ \\
\hline & & $2.08, \mathrm{~m}$ & & $1.98, \mathrm{~m}$ \\
\hline $2^{\prime}$ & $20.8, \mathrm{CH}$ & $1.96, \mathrm{~s}$ & & \\
\hline $2^{\prime \prime}$ & $20.9, \mathrm{CH}_{3}$ & $2.03, \mathrm{~s}^{a}$ & & \\
\hline 3 & $70.3, \mathrm{CH}$ & $5.004, \mathrm{~m}^{a}$ & $121.3, \mathrm{CH}$ & 5.35, brs \\
\hline 4 & $140.5, \mathrm{C}$ & & 133.5, C & \\
\hline 5 & $52.5, \mathrm{CH}$ & $1.67, \mathrm{~d}(10.8)$ & $51.1, \mathrm{CH}$ & $2.34, \mathrm{~d}(11.6)^{a}$ \\
\hline 6 & $77.8, \mathrm{CH}$ & $4.95, \mathrm{~d}(10.8)$ & $81.5, \mathrm{CH}$ & $3.94, \mathrm{~m}^{a}$ \\
\hline 7 & $172.3, \mathrm{C}$ & & $51.0, \mathrm{C}$ & $2.49, \mathrm{~m}$ \\
\hline \multirow[t]{2}{*}{8} & $22.5, \mathrm{CH}_{2}$ & $2.37, \operatorname{td}(14.0,5.5)$ & $21.2, \mathrm{CH}_{2}$ & $1.64, \mathrm{qd}(12.5,3.0)$ \\
\hline & & 2.94, brdd $(14.6,2.8)$ & & $2.08, \mathrm{~m}^{a}$ \\
\hline \multirow[t]{2}{*}{9} & $36.3, \mathrm{CH}_{2}$ & 2.06, brdd $(13.3,5.0)^{a}$ & $34.2, \mathrm{CH}_{2}$ & $2.07, \mathrm{~m}^{a}$ \\
\hline & & 2.21, ddd $(12.8,5.8,1.8)$ & & $1.31, \operatorname{td}(13.2,4.1)$ \\
\hline 10 & $41.3, \mathrm{C}$ & & $40.9, \mathrm{C}$ & \\
\hline 11 & $119.4, \mathrm{C}$ & & $170.8, \mathrm{C}$ & \\
\hline 12 & $168.3, \mathrm{C}$ & & $138.9, \mathrm{C}$ & \\
\hline \multirow[t]{2}{*}{13} & $54.8, \mathrm{CH}_{2}$ & $4.68, \mathrm{~d}(2.4)$ & $116.9, \mathrm{CH}_{2}$ & $5.40, \mathrm{~d}(3.8)$ \\
\hline & & & & $6.07, \mathrm{~d}(3.1)$ \\
\hline 14 & $10.4, \mathrm{CH}_{3}$ & $0.83, \operatorname{td}(13.5,4.4)$ & $11.0, \mathrm{CH}_{3}$ & $0.88, \mathrm{~s}$ \\
\hline \multirow[t]{2}{*}{15} & $107.9, \mathrm{CH}_{3}$ & $5.003, \mathrm{~s}^{a}$ & 23.3, $\mathrm{CH}_{3}$ & $1.84, \mathrm{~s}$ \\
\hline & & $5.12, \mathrm{~s}$ & & \\
\hline${ }^{a}$ Overlapp & signals & & & \\
\hline
\end{tabular}


Table S4. ${ }^{1} \mathrm{H}$ and ${ }^{13} \mathrm{C}$ NMR Spectroscopic Data $\left(500 \mathrm{MHz}, \mathrm{CDCl}_{3}\right)$ for compounds 12 and 16.

\begin{tabular}{|c|c|c|c|c|}
\hline \multirow[b]{2}{*}{ position } & \multicolumn{2}{|r|}{12} & \multicolumn{2}{|r|}{16} \\
\hline & $\delta \mathrm{C}$, type & $\delta \mathrm{H}(\mathrm{J}$ in $\mathrm{Hz})$ & $\delta \mathrm{C}$, type & $\overline{\delta \mathrm{H}(\mathrm{J} \text { in } \mathrm{Hz})}$ \\
\hline 2 & $162.7, \mathrm{C}$ & & $163.4, \mathrm{C}$ & \\
\hline 3 & $101.8, \mathrm{CH}$ & $6.76, \mathrm{~s}$ & 103.4, CH & $6.79, \mathrm{~s}$ \\
\hline 4 & 181.2, C & & $182.3, \mathrm{C}$ & \\
\hline $4 a$ & $103.0, \mathrm{C}$ & & 104.2, C & \\
\hline 5 & $151.8, \mathrm{C}$ & & $152.8, \mathrm{C}$ & \\
\hline 6 & $130.5, \mathrm{C}$ & & 131.4, C & \\
\hline 7 & $156.8, \mathrm{C}$ & & 157.3, C & \\
\hline 8 & 93.4, CH & $6.57, \mathrm{~s}$ & $94.4, \mathrm{CH}$ & $6.56, \mathrm{~s}$ \\
\hline $8 \mathrm{a}$ & 151.6, C & & $152.5, \mathrm{C}$ & \\
\hline $1^{\prime}$ & 120.6, C & & $122.9, \mathrm{C}$ & \\
\hline $2^{\prime}$ & $109.2, \mathrm{CH}$ & $7.48, \mathrm{~m}^{a}$ & $109.4, \mathrm{CH}$ & $7.44, \mathrm{~d}(2.0)$ \\
\hline $3^{\prime}$ & 147.1, C & & $149.0, \mathrm{CH}$ & \\
\hline $4^{\prime}$ & $149.8, \mathrm{C}$ & & $152.2, \mathrm{C}$ & \\
\hline $5^{\prime}$ & $114.8, \mathrm{CH}$ & $6.93, \mathrm{~d}(8.8)$ & 111.7, C & $7.02, \mathrm{~d}(8.5)$ \\
\hline $6^{\prime}$ & $119.4, \mathrm{CH}$ & $7.49, \mathrm{~m}^{a}$ & $120.1, \mathrm{CH}$ & $7.55, \mathrm{dd}(8.5,2.0)$ \\
\hline OCH3-6 & $59.0, \mathrm{CH}_{3}$ & $3.77, \mathrm{~s}$ & $60.0, \mathrm{CH}_{3}$ & $3.77, \mathrm{~s}$ \\
\hline OCH3-3' & $55.0, \mathrm{CH}_{3}$ & $3.89, \mathrm{~s}$ & $55.9, \mathrm{CH}_{3}$ & $3.85, \mathrm{~s}$ \\
\hline OCH3-4' & & & $55.8, \mathrm{CH}_{3}$ & $3.82, \mathrm{~s}$ \\
\hline OH-5 & & $12.97, \mathrm{brs}$ & & $12.97, \mathrm{brs}$ \\
\hline${ }^{a}$ Overlappi & signals & & & \\
\hline
\end{tabular}


Table S5. ${ }^{1} \mathrm{H}$ and ${ }^{13} \mathrm{C}$ NMR Spectroscopic Data $\left(500 \mathrm{MHz}, \mathrm{CDCl}_{3}\right.$ ) for compounds 13 - 15.

\begin{tabular}{|c|c|c|c|c|c|c|}
\hline \multirow[b]{2}{*}{ position } & \multicolumn{2}{|r|}{$13^{b}$} & \multicolumn{2}{|r|}{$14^{b}$} & \multicolumn{2}{|r|}{$15^{c}$} \\
\hline & $\delta_{\mathrm{C}}$, type & $\delta_{\mathrm{H}}(J$ in $\mathrm{Hz})$ & $\delta_{\mathrm{C}}$, type & $\delta_{\mathrm{H}}(J$ in $\mathrm{Hz})$ & $\delta_{\mathrm{C}}$, type & $\delta_{\mathrm{H}}(J$ in $\mathrm{Hz})$ \\
\hline 1 & $81.8, \mathrm{C}$ & & $81.8, \mathrm{C}$ & & 136.3, C & \\
\hline $2 \mathrm{a}$ & $40.9, \mathrm{CH}_{2}$ & $2.21, \operatorname{brd}(15.3)^{a}$ & $40.9, \mathrm{CH}_{2}$ & $2.21, \operatorname{brd}(15.3)^{a}$ & $33.0, \mathrm{CH}_{2}$ & $2.63, \mathrm{~d}(17.7)$ \\
\hline $2 b$ & & 1.89, bd (15.3) & & 1.89, brd (15.3) & & $2.41^{a}$ \\
\hline 3 & $64.3, \mathrm{CH}$ & 3.54 , brs & $64.3, \mathrm{CH}$ & 3.54 , brs & $63.1, \mathrm{CH}$ & $3.32, \mathrm{~s}$ \\
\hline 4 & $67.4, \mathrm{C}$ & & $67.4, \mathrm{C}$ & & $66.5, \mathrm{C}$ & \\
\hline 5 & $61.0, \mathrm{CH}$ & $2.33, \mathrm{dd}(11.3,3.1)$ & $61.0, \mathrm{CH}$ & $2.33, \mathrm{dd}(11.3,3.1)$ & $51.2, \mathrm{CH}$ & $3.01, \mathrm{~d}(10.7)$ \\
\hline 6 & $75.2, \mathrm{CH}$ & 3.92, ddd $(11.1,9.1,6.4)$ & $75.2, \mathrm{CH}$ & 3.92, ddd $(11.1,9.1,6.4)$ & 77.7, $\mathrm{CH}$ & $3.64, \mathrm{dd}(10.2,10.2)$ \\
\hline 7 & $46.8, \mathrm{CH}$ & $3.23, \mathrm{~m}$ & $46.8, \mathrm{CH}$ & $3.23, \mathrm{~m}$ & $56.1, \mathrm{CH}$ & $\begin{array}{l}3.06, \operatorname{dddd}(10.2,10.2,2.9 \text {, } \\
2.9)\end{array}$ \\
\hline 8 & $73.1, \mathrm{CH}$ & $4.95, \mathrm{~m}$ & $73.1, \mathrm{CH}$ & $4.95, \mathrm{~m}$ & $70.1, \mathrm{CH}$ & 4.71, ddd $(10.7,10.7,1.8)$ \\
\hline $9 \mathrm{a}$ & $35.4, \mathrm{CH}_{2}$ & 2.55, ddd $(15.2,7.3,2.9)$ & $35.4, \mathrm{CH}_{2}$ & 2.55, ddd $(15.2,7.3,2.9)$ & $41.2, \mathrm{CH}_{2}$ & $2.42^{a}$ \\
\hline $9 b$ & & $2.26, \mathrm{~m}^{a}$ & & $2.26, \mathrm{~m}^{a}$ & & $2.15, \mathrm{dd}(13.6,2.0)$ \\
\hline 10 & $140.7, \mathrm{C}$ & & $140.7, \mathrm{C}$ & & 127.9, C & \\
\hline 11 & 168.7, C & & 168.7, C & & 168.3, C & \\
\hline 12 & 136.6, C & & 136.6, C & & $136.8, \mathrm{C}$ & \\
\hline $13 \mathrm{a}$ & $122.7, \mathrm{CH}_{2}$ & $6.18, \mathrm{~d}(3.4)$ & $122.7, \mathrm{CH}_{2}$ & $6.18, \mathrm{~d}(3.4)$ & $120.1, \mathrm{CH}_{2}$ & $6.07, \mathrm{~d}(3.0)$ \\
\hline $13 b$ & & $5.60, \mathrm{dd}(4.7,3.2)^{d}$ & & $5.60, \mathrm{dd}(4.7,3.2)^{d}$ & & $5.48, \mathrm{~d}(3.0)$ \\
\hline 14 & $18.6, \mathrm{CH}_{3}$ & $1.63, \mathrm{~s}$ & $18.6, \mathrm{CH}_{3}$ & $1.63, \mathrm{~s}$ & $18.6, \mathrm{CH}_{3}$ & $1.59, \mathrm{~s}$ \\
\hline \multirow[t]{2}{*}{15} & $118.0, \mathrm{CH}_{3}$ & 5.53, brd (3.4) & $118.0, \mathrm{CH}_{3}$ & 5.53, brd (3.4) & $21.9, \mathrm{CH}_{3}$ & $1.66, \mathrm{~s}$ \\
\hline & & 4.98, brs & & 4.98, brs & & \\
\hline $1^{\prime}$ & 175.9, C & & $172.4, \mathrm{C}$ & & $169.4, \mathrm{C}$ & \\
\hline $2^{\prime}$ & $41.5, \mathrm{CH}$ & $2.42, \mathrm{ddq}(13.9,13.9,7.0)$ & $43.5, \mathrm{CH}_{2}$ & $2.25, \mathrm{~m}^{a}$ & $20.7, \mathrm{CH}_{3}$ & $2.04, \mathrm{~s}$ \\
\hline $3{ }^{\prime} \mathrm{a}$ & $26.4, \mathrm{CH}_{2}$ & $1.73, \mathrm{~m}$ & $25.6, \mathrm{CH}$ & 2.14 , tqq $(6.7,6.7,6.7)$ & & \\
\hline $3^{\prime} b$ & & $1.49, \mathrm{~m}$ & & & & \\
\hline $4^{\prime}$ & $11.8, \mathrm{CH}_{3}$ & $0.94, \mathrm{dd}(7.5,7.5)$ & $22.43, \mathrm{CH}_{3}$ & $0.98, \mathrm{~d}(6.7)^{a}$ & & \\
\hline $5^{\prime}$ & $16.8, \mathrm{CH}_{3}$ & $1.19, \mathrm{~d}(7.0)$ & $22.46, \mathrm{CH}_{3}$ & $0.99, \mathrm{~d}(6.7)^{a}$ & & \\
\hline
\end{tabular}


Table S6. ${ }^{1} \mathrm{H}$ and ${ }^{13} \mathrm{C}$ NMR Spectroscopic Data (500 MHz, $\mathrm{CDCl}_{3}$ ) for compound 18.

\begin{tabular}{|c|c|c|c|}
\hline \multirow[b]{2}{*}{ position } & \multicolumn{3}{|c|}{18} \\
\hline & $\overline{\delta_{\mathrm{C}}, \text { type }}$ & $\overline{\delta_{\mathrm{H}}(J \text { in } \mathrm{Hz})}$ & HMBC \\
\hline 1 & 134.1, C & & \\
\hline 2 & $194.8, \mathrm{C}$ & & \\
\hline 3 & $136.2, \mathrm{CH}$ & $6.15, \mathrm{~s}$ & $1,2,4,5,14$ \\
\hline 4 & $170.1, \mathrm{C}$ & & \\
\hline 5 & $51.9, \mathrm{CH}$ & $3.26, \mathrm{~d}(10.0)$ & $1,4,6,7$ \\
\hline 6 & $80.1, \mathrm{CH}$ & $3.99, \mathrm{dd}(10.4,10.4)$ & 1,7 \\
\hline 7 & $59.2, \mathrm{CH}$ & $2.72, \mathrm{dd}(10.8,10.8)$ & $4^{\prime}, 5,6,8,9,12$ \\
\hline 8 & $68.3, \mathrm{CH}$ & $4.75, \operatorname{ddd}(10.6,10.6,1.7)$ & $1 ", 7$ \\
\hline $9 \mathrm{a}$ & $44.5, \mathrm{CH}_{2}$ & $2.48, \mathrm{dd}(12.7,10.8)$ & $1,7,8,15$ \\
\hline $9 b$ & & $2.28, \mathrm{~m}^{a}$ & $1,7,8,15$ \\
\hline 10 & $143.8, \mathrm{C}$ & & \\
\hline 11 & 178.6, C & & \\
\hline 12 & $61.3, \mathrm{C}$ & & \\
\hline $13 \mathrm{a}$ & $40.2, \mathrm{CH}_{2}$ & $2.63, \mathrm{~d}(11.6)$ & $1^{\prime}, 2^{\prime}, 4^{\prime}, 10^{\prime}, 11$ \\
\hline $13 b$ & & $1.86, \mathrm{~d}(11.9)$ & $1^{\prime}, 2^{\prime}, 5^{\prime}, 7,11$ \\
\hline 14 & $20.2, \mathrm{CH}_{3}$ & $2.28, \mathrm{~s}^{a}$ & $3,4,5$ \\
\hline 15 & $20.5, \mathrm{CH}_{3}$ & $2.36, \mathrm{~s}$ & $1,9,10$ \\
\hline $1^{\prime}$ & $63.0, \mathrm{C}$ & & \\
\hline $2^{\prime}$ & $137.5, \mathrm{CH}$ & $5.85, \mathrm{~d}(5.8)$ & $1^{\prime}, 3^{\prime}$ \\
\hline $3^{\prime}$ & $136.4, \mathrm{CH}$ & $5.81, \mathrm{~d}(5.5)$ & $1^{\prime}, 2^{\prime}, 5^{\prime}, 12,14^{\prime}$ \\
\hline $4^{\prime}$ & $57.9, \mathrm{C}$ & & \\
\hline $5^{\prime}$ & $66.9, \mathrm{CH}$ & $3.04, \mathrm{~d}(9.8)$ & $1^{\prime}, 2^{\prime}, 6^{\prime}, 7^{\prime}$ \\
\hline $6^{\prime}$ & $79.2, \mathrm{CH}$ & $3.96, \mathrm{dd}(9.6,9.6)$ & $5^{\prime}, 8^{\prime}, 12$ \\
\hline $7^{\prime}$ & $43.1, \mathrm{CH}$ & $3.30, \mathrm{~m}$ & $6^{\prime}$ \\
\hline $8^{\prime} \mathrm{a}$ & 23.5, $\mathrm{CH}_{2}$ & $2.22, \mathrm{~m}$ & $9^{\prime}$ \\
\hline $8^{\prime} \mathrm{b}$ & & $1.38, \mathrm{~m}$ & $7^{\prime}$ \\
\hline $9^{\prime} \mathrm{a}$ & $34.6, \mathrm{CH}_{2}$ & $1.82, \mathrm{~m}$ & $1^{\prime}, 7^{\prime}, 8^{\prime}, 10^{\prime}, 15^{\prime}$ \\
\hline $9^{\prime} \mathrm{b}$ & & $1.75, \mathrm{~m}$ & $8^{\prime}$ \\
\hline $10^{\prime}$ & $72.5, \mathrm{C}$ & & \\
\hline $11^{\prime}$ & $170.4, \mathrm{C}$ & & \\
\hline $12^{\prime}$ & 140.6, C & & \\
\hline \multirow[t]{2}{*}{$13^{\prime}$} & $118.8, \mathrm{CH}_{2}$ & $6.01, \mathrm{~d}(3.4)$ & $7^{\prime}, 11^{\prime}, 12^{\prime}$ \\
\hline & & $5.29, \mathrm{~d}(3.0)$ & $7^{\prime}, 11^{\prime}, 12^{\prime}$ \\
\hline $14^{\prime}$ & 17.0, $\mathrm{CH}_{3}$ & $1.44, \mathrm{~s}$ & $3^{\prime}, 4^{\prime}, 5^{\prime}, 12$ \\
\hline $15^{\prime}$ & $29.8, \mathrm{CH}_{3}$ & $1.27, \mathrm{~s}$ & $1^{\prime}, 8^{\prime}, 9^{\prime}, 10^{\prime}$ \\
\hline $1 "$ & 168.9, C & & \\
\hline $2^{\prime \prime}$ & 21.6, $\mathrm{CH}_{3}$ & $1.96, \mathrm{~s}$ & $1 ", 8$ \\
\hline
\end{tabular}



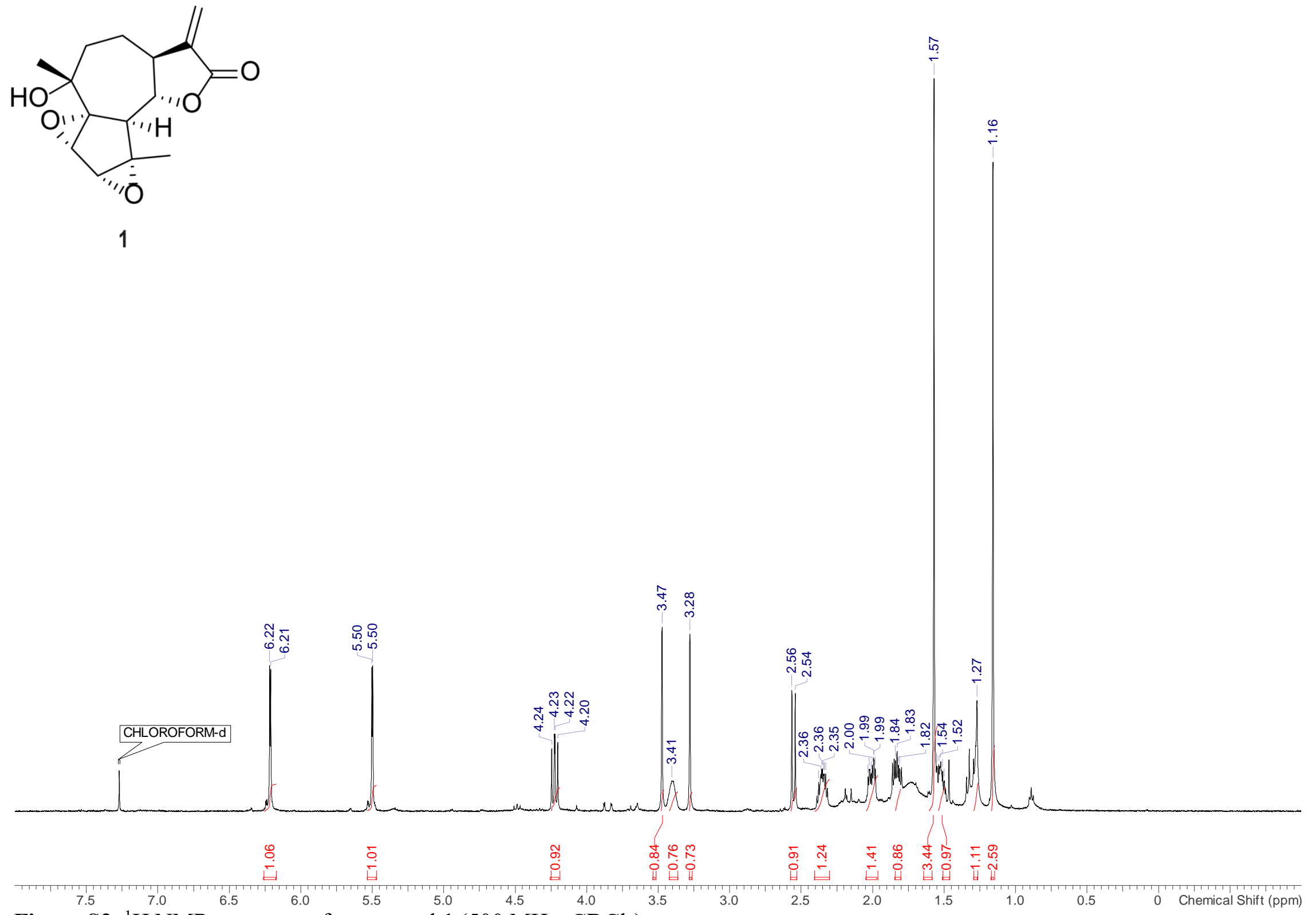

Figure S3. ${ }^{1} \mathrm{H}$ NMR spectrum of compound 1 (500 MHz, $\left.\mathrm{CDCl}_{3}\right)$. 


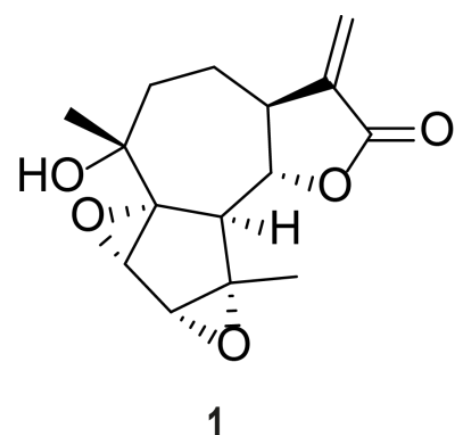

CHLOROFORM-d
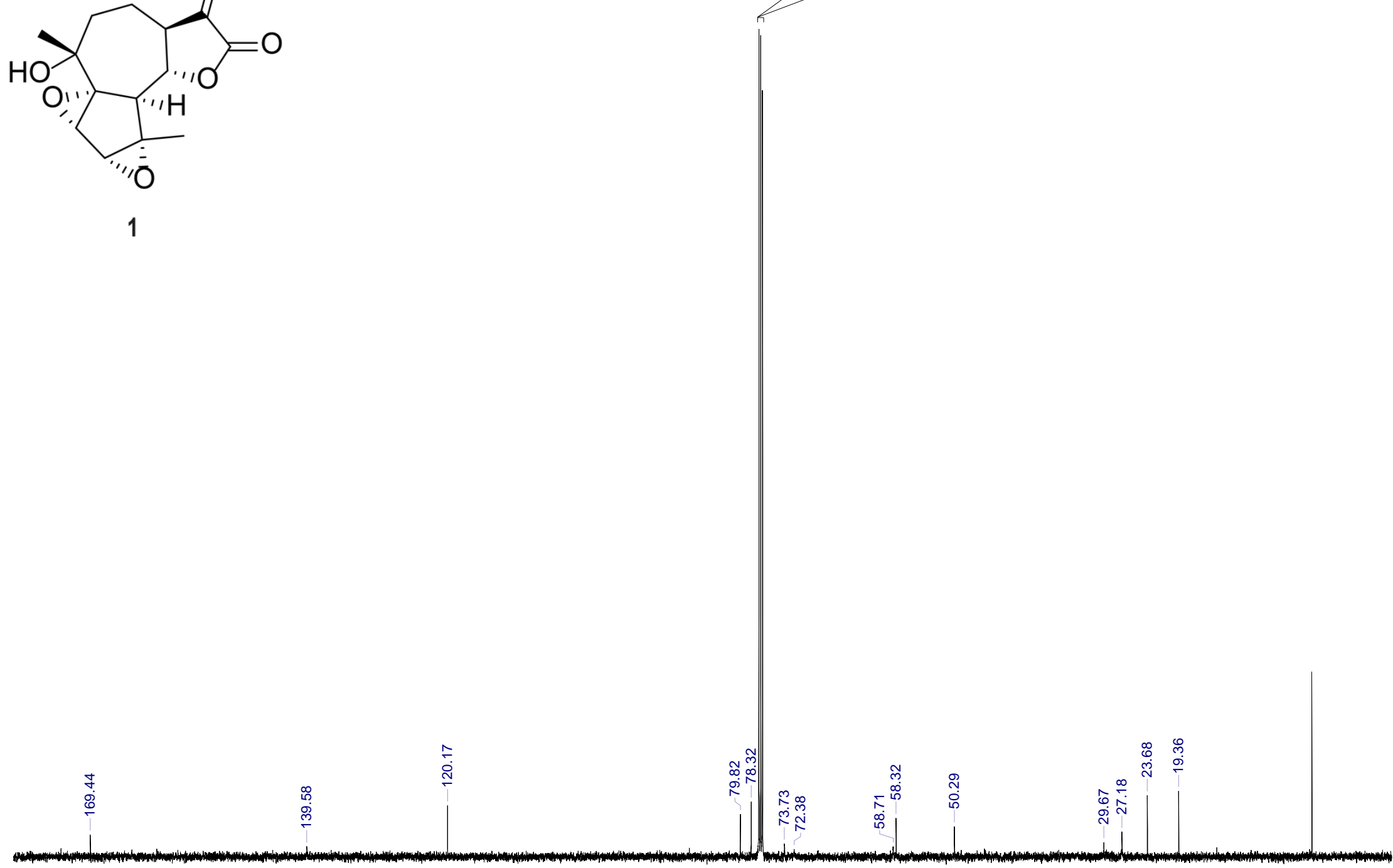

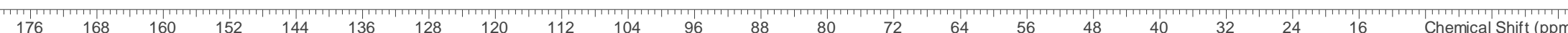

Figure S4. ${ }^{13} \mathrm{C}$ spectrum of compound $\mathbf{1}\left(125 \mathrm{MHz}, \mathrm{CDCl}_{3}\right)$. 


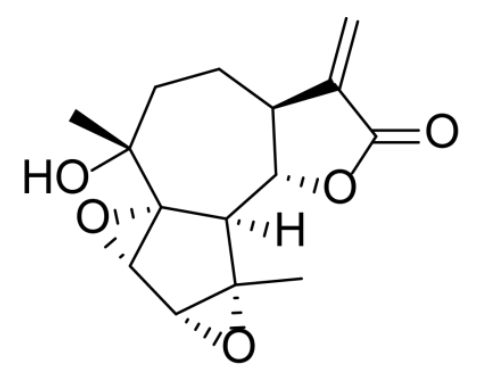

CHLOROFORM-d

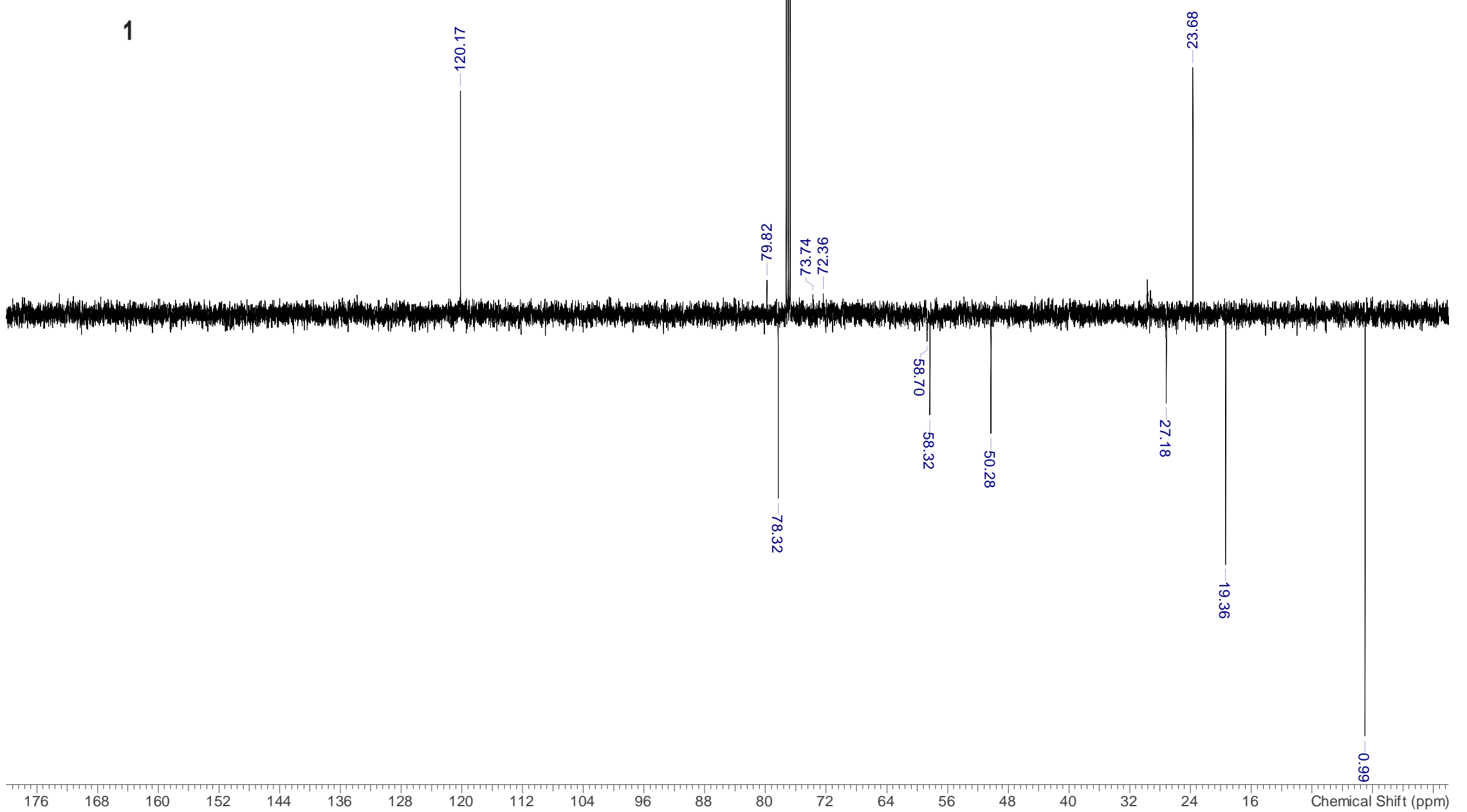

Figure S5. ${ }^{13} \mathrm{C}$-DEPTq spectrum of compound $\mathbf{1}\left(125 \mathrm{MHz}, \mathrm{CDCl}_{3}\right)$. 


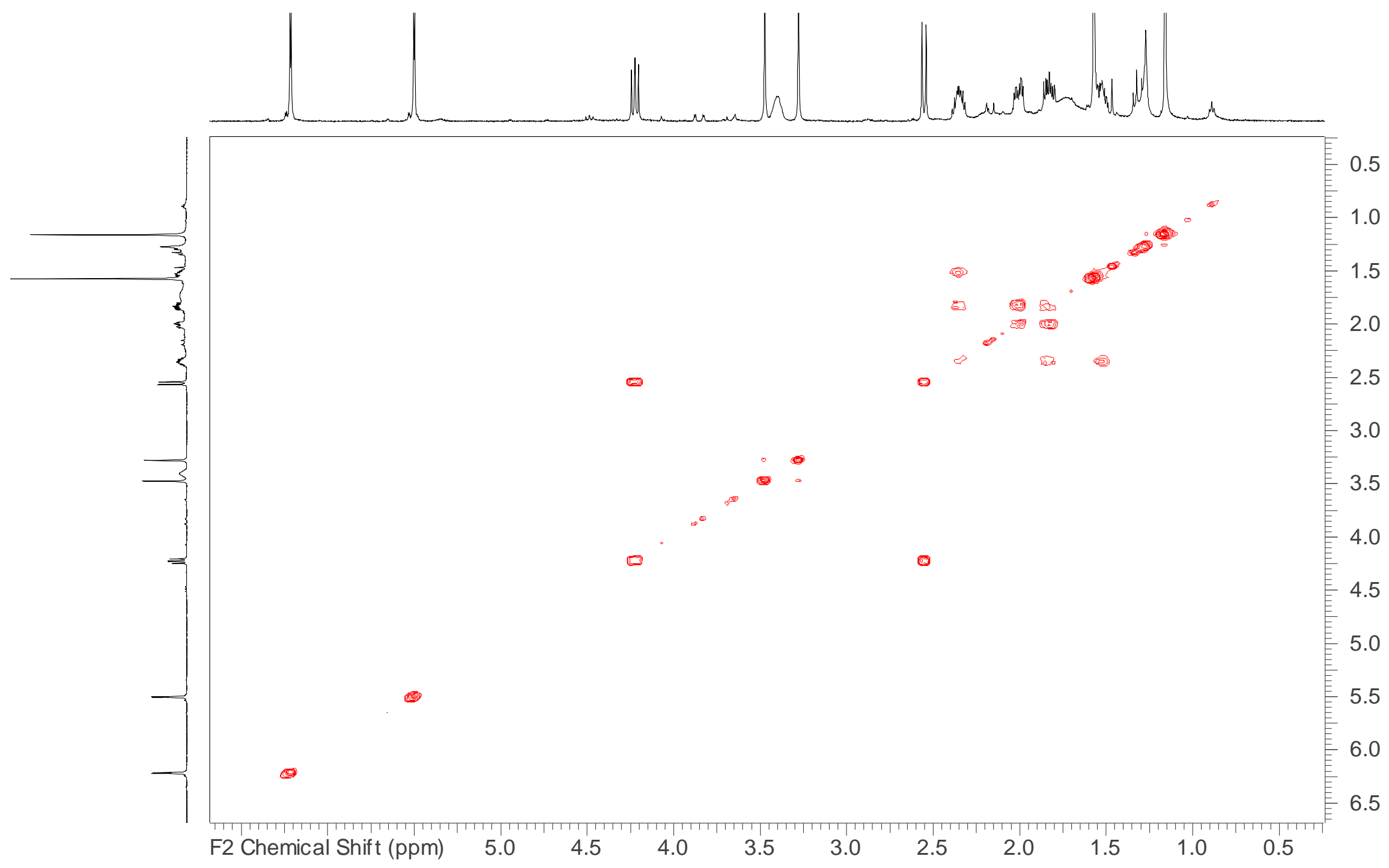

Figure S6. ${ }^{1} \mathrm{H}-{ }^{1} \mathrm{H}$ COSY spectrum of compound $1\left(500 \mathrm{MHz}, \mathrm{CDCl}_{3}\right)$. 


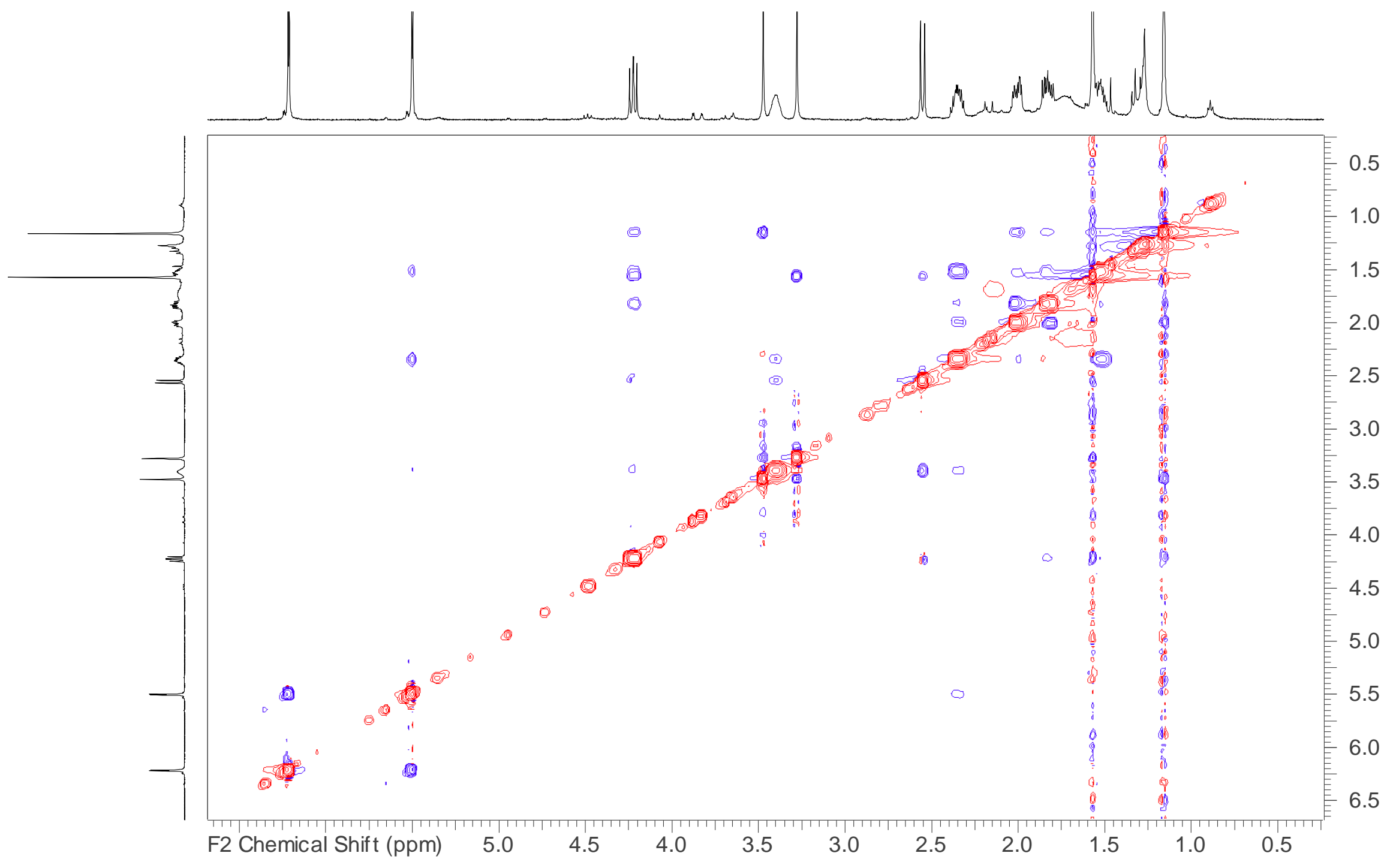

Figure S7. . ${ }^{1} \mathrm{H}-{ }^{1} \mathrm{H}$ NOESY spectrum of compound $1\left(500 \mathrm{MHz}, \mathrm{CDCl}_{3}\right)$. 


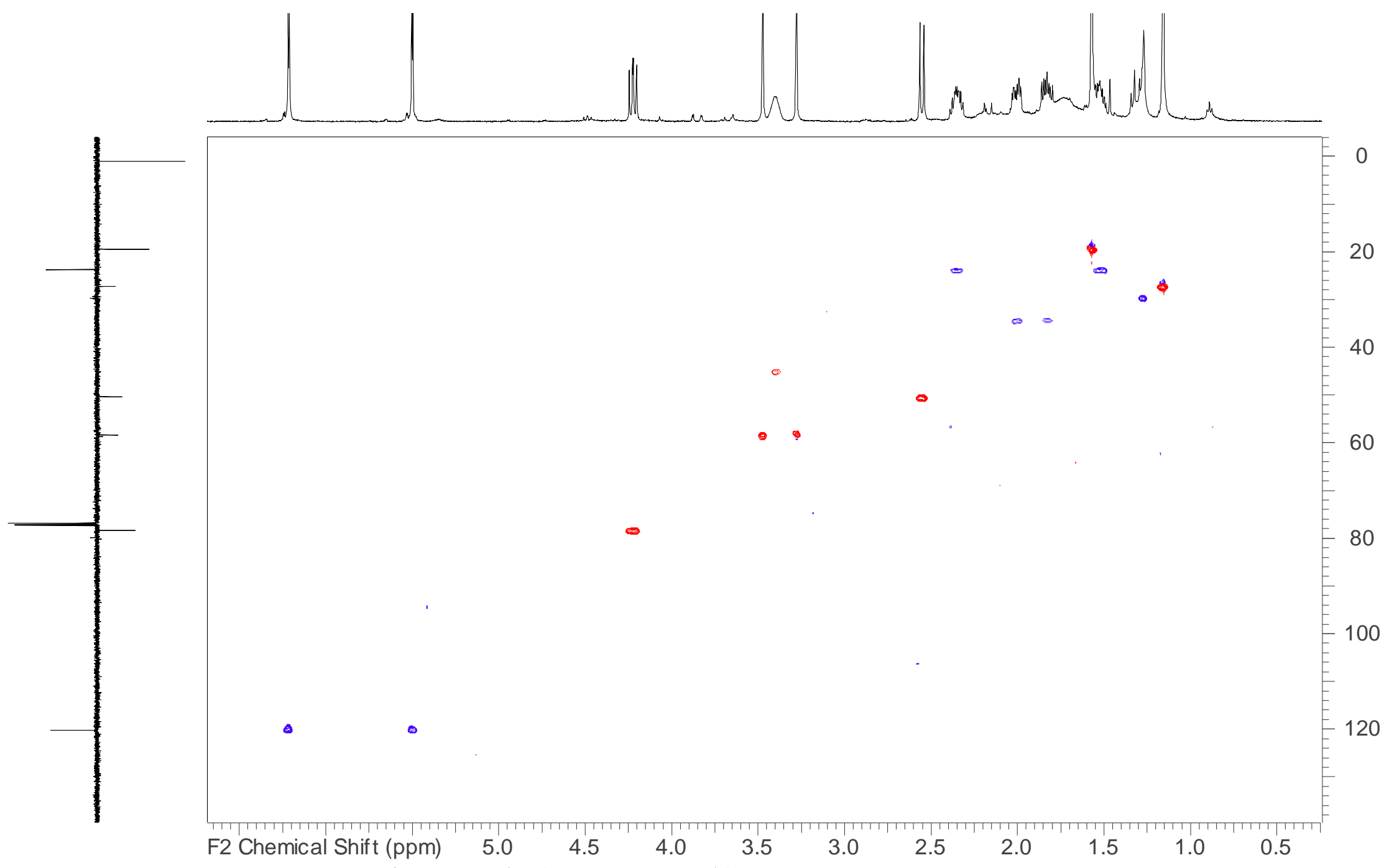

Figure S8. HSQC-DEPT spectrum of compound 1 (500 MHz, $\mathrm{CDCl}_{3}$ ). 


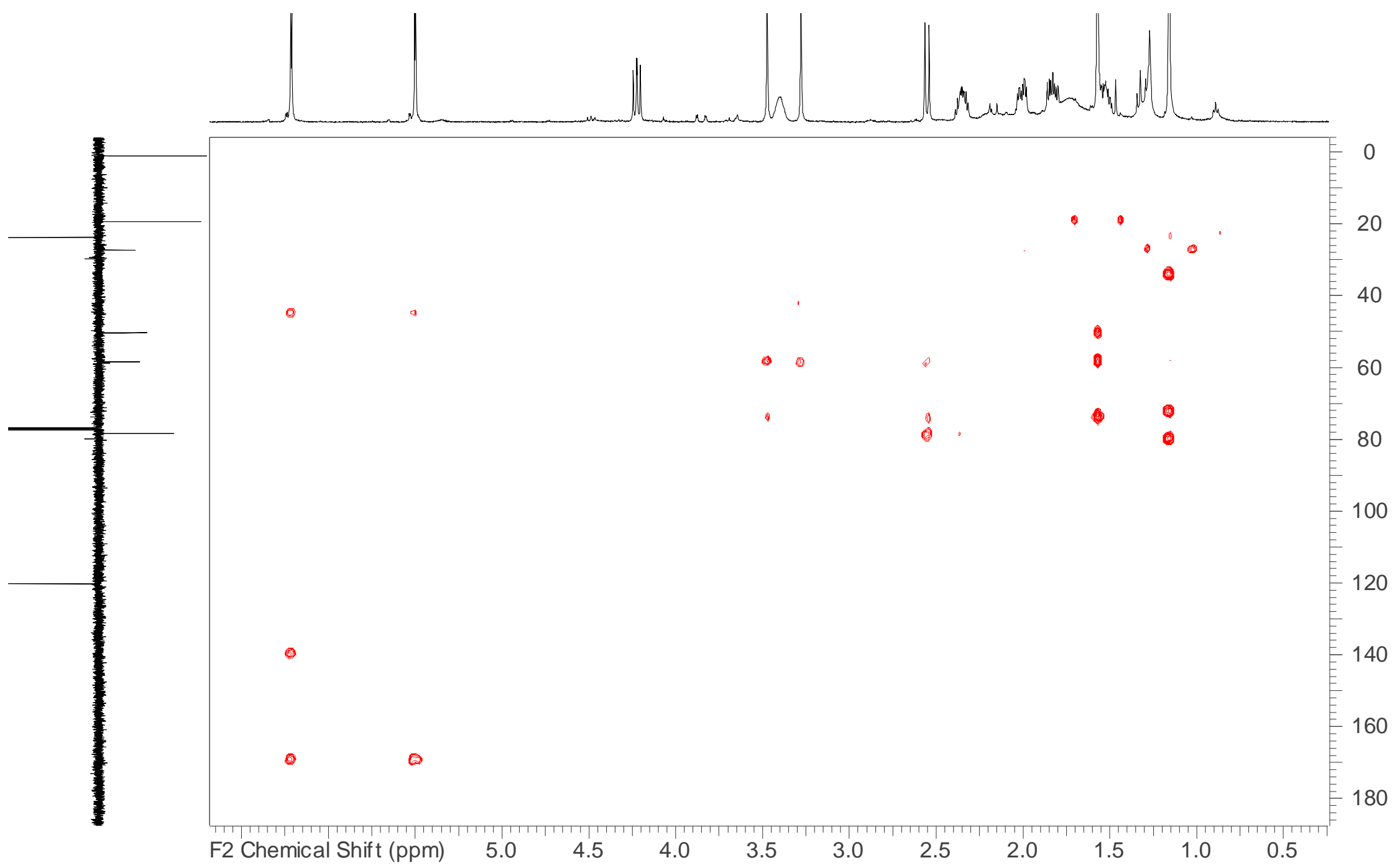

Figure S9. HMBC spectrum of compound $1\left(500 \mathrm{MHz}, \mathrm{CDCl}_{3}\right)$. 


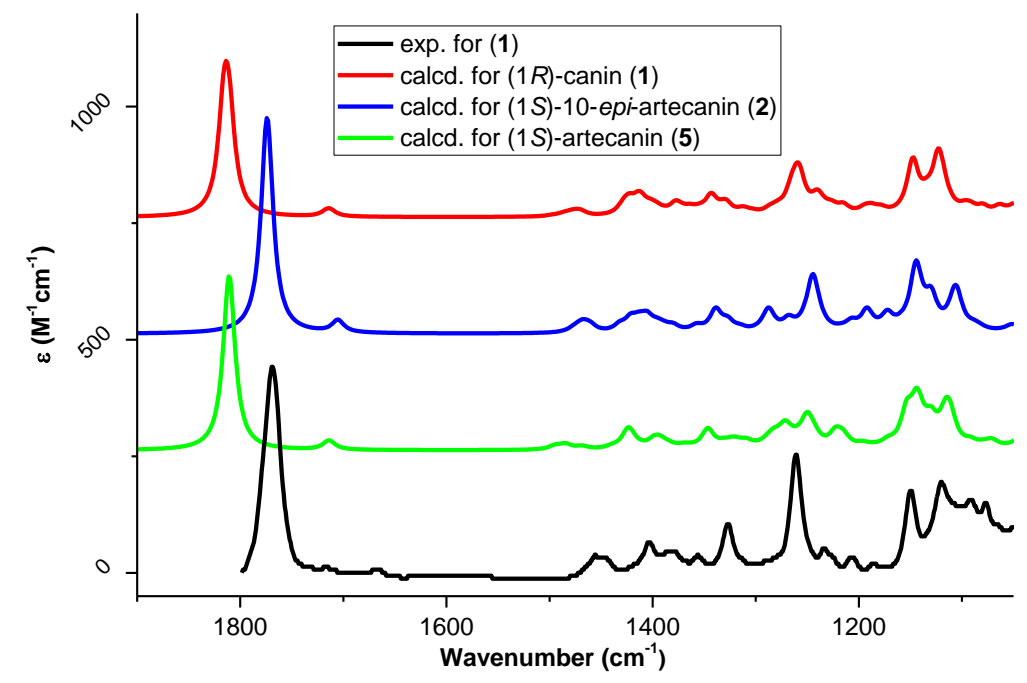

Figure S10. Comparison of experimental and computed IR spectra in chloroform for compound $\mathbf{1}$ in the region 1900-1050 $\mathrm{cm}^{-1}$. Computed spectra (compounds $\mathbf{1}, \mathbf{2}$ and 5) were scaled according to the overall maximal SimVCD value (0.9865). 


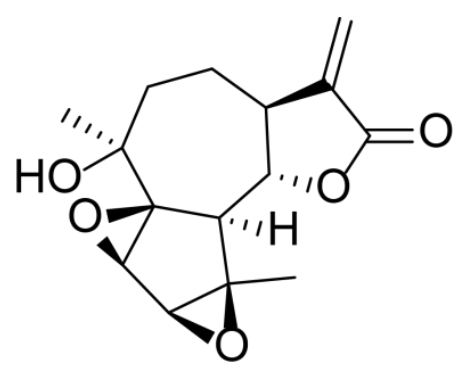

2

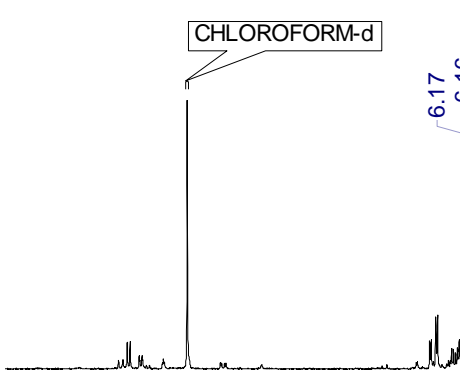

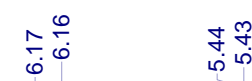
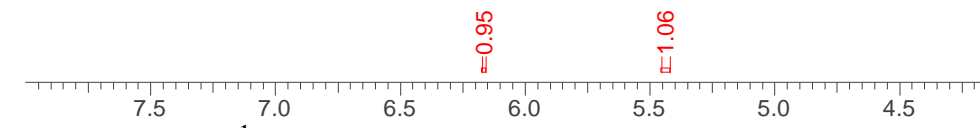

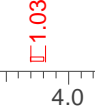

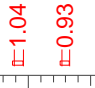

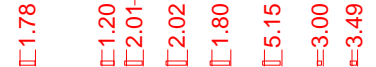

Figure S11. ${ }^{1} \mathrm{H}$ NMR spectrum of compound $2\left(500 \mathrm{MHz}, \mathrm{CDCl}_{3}\right)$. 


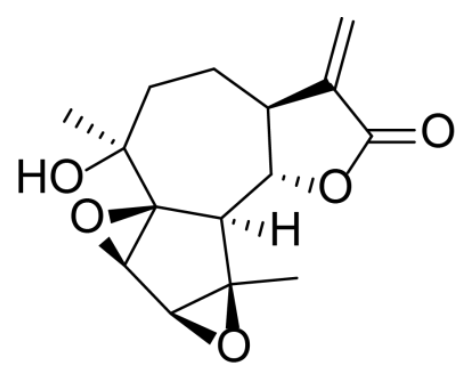

2

CHLOROFORM-d

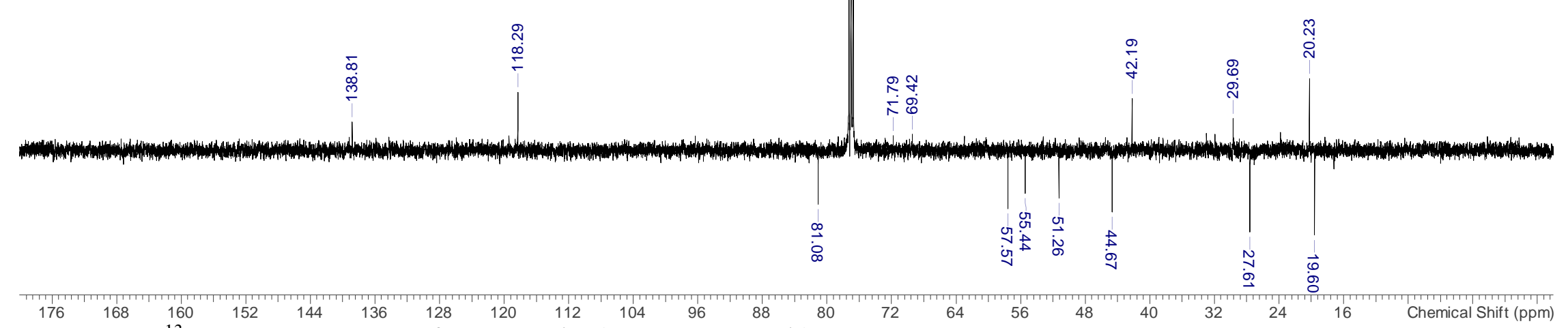

Figure S12. ${ }^{13} \mathrm{C}$-DEPTq spectrum of compound $2\left(125 \mathrm{MHz}, \mathrm{CDCl}_{3}\right)$. 


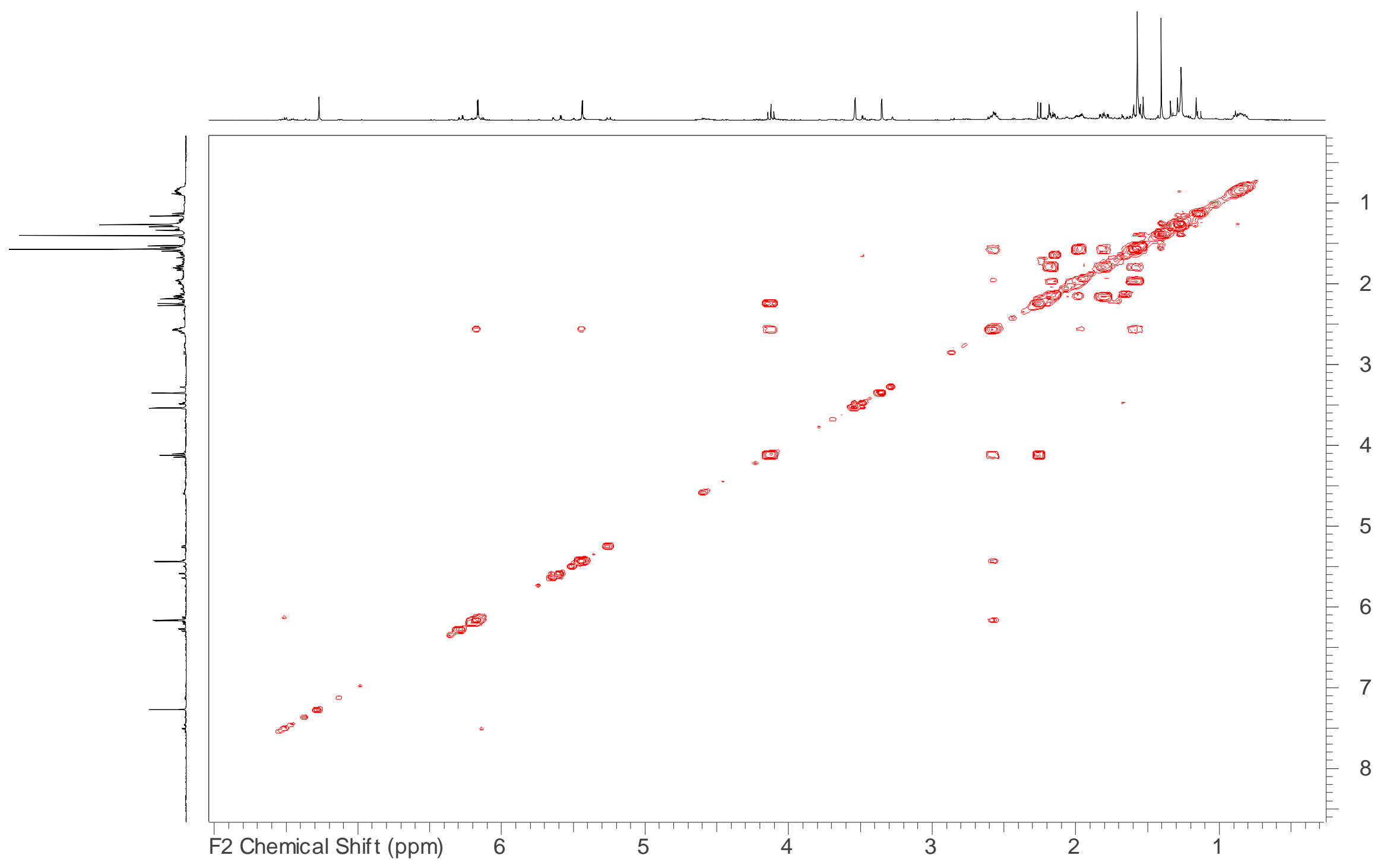

Figure S13. ${ }^{1} \mathrm{H}-{ }^{1} \mathrm{H}$ COSY spectrum of compound $2\left(500 \mathrm{MHz}, \mathrm{CDCl}_{3}\right)$. 


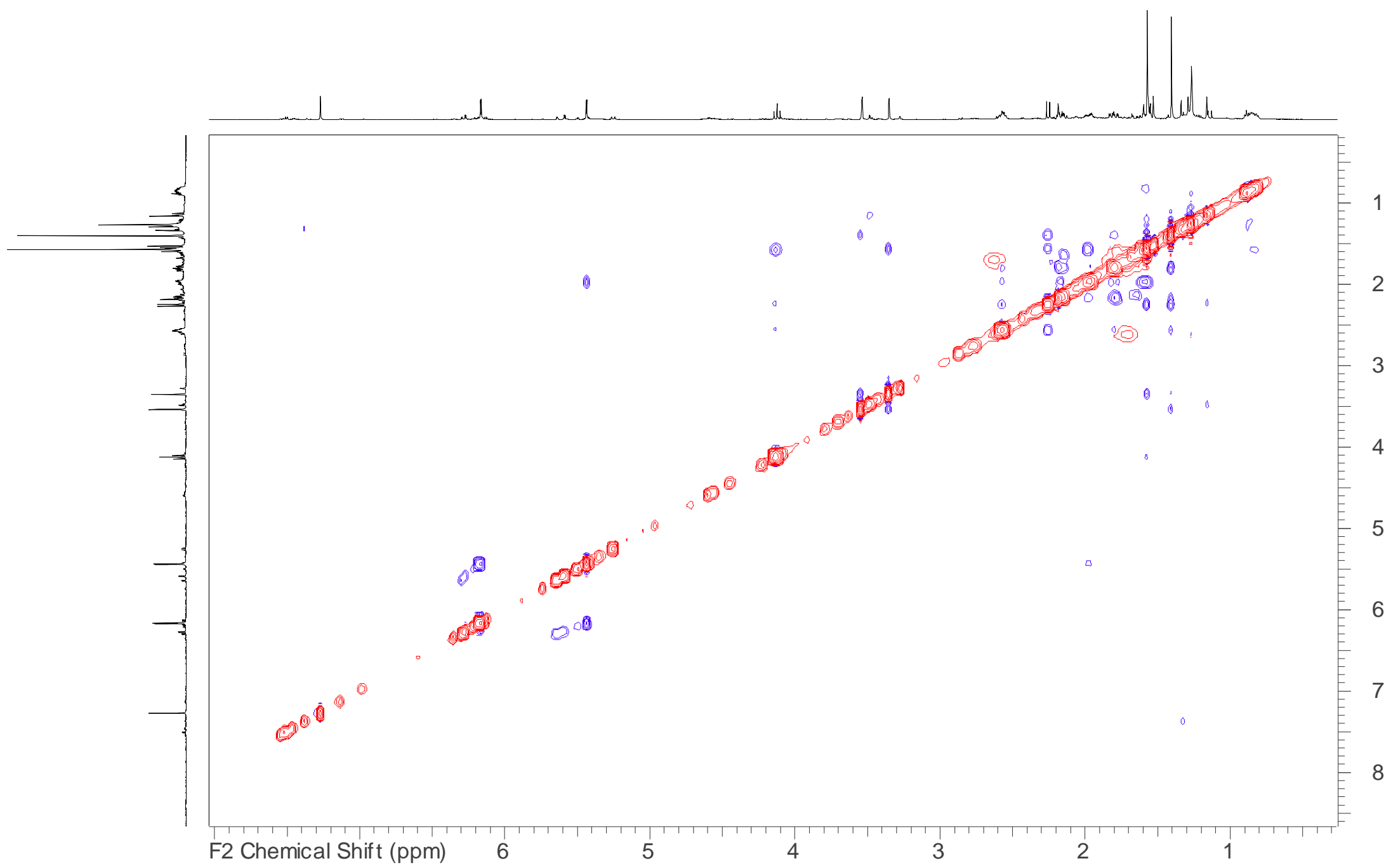

Figure S14. ${ }^{1} \mathrm{H}-{ }^{1} \mathrm{H}$ NOESY spectrum of compound $2\left(500 \mathrm{MHz}, \mathrm{CDCl}_{3}\right)$.

24 


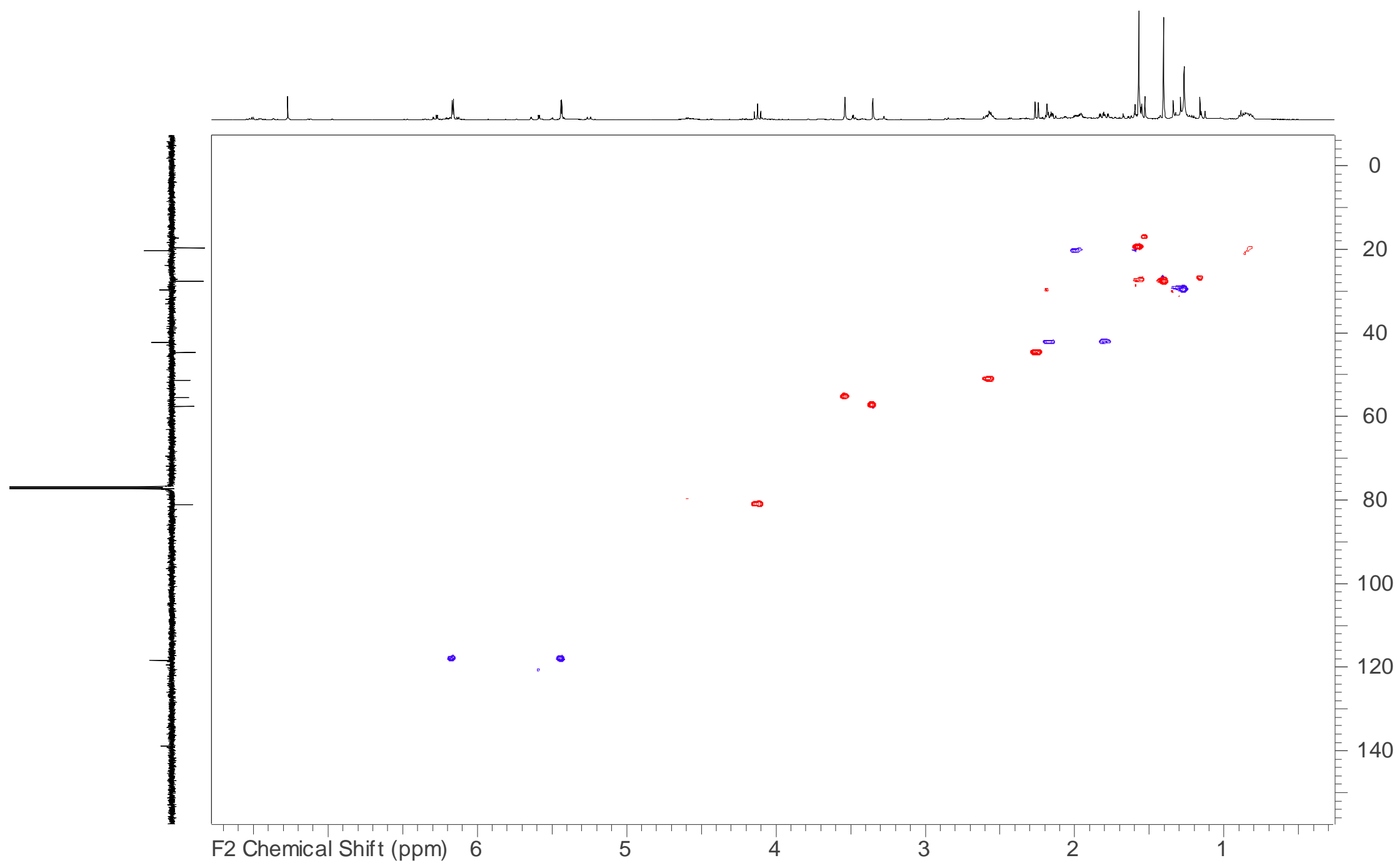

Figure S15. HSQC-DEPT spectrum of compound 2 (500 MHz, $\mathrm{CDCl}_{3}$ ). 


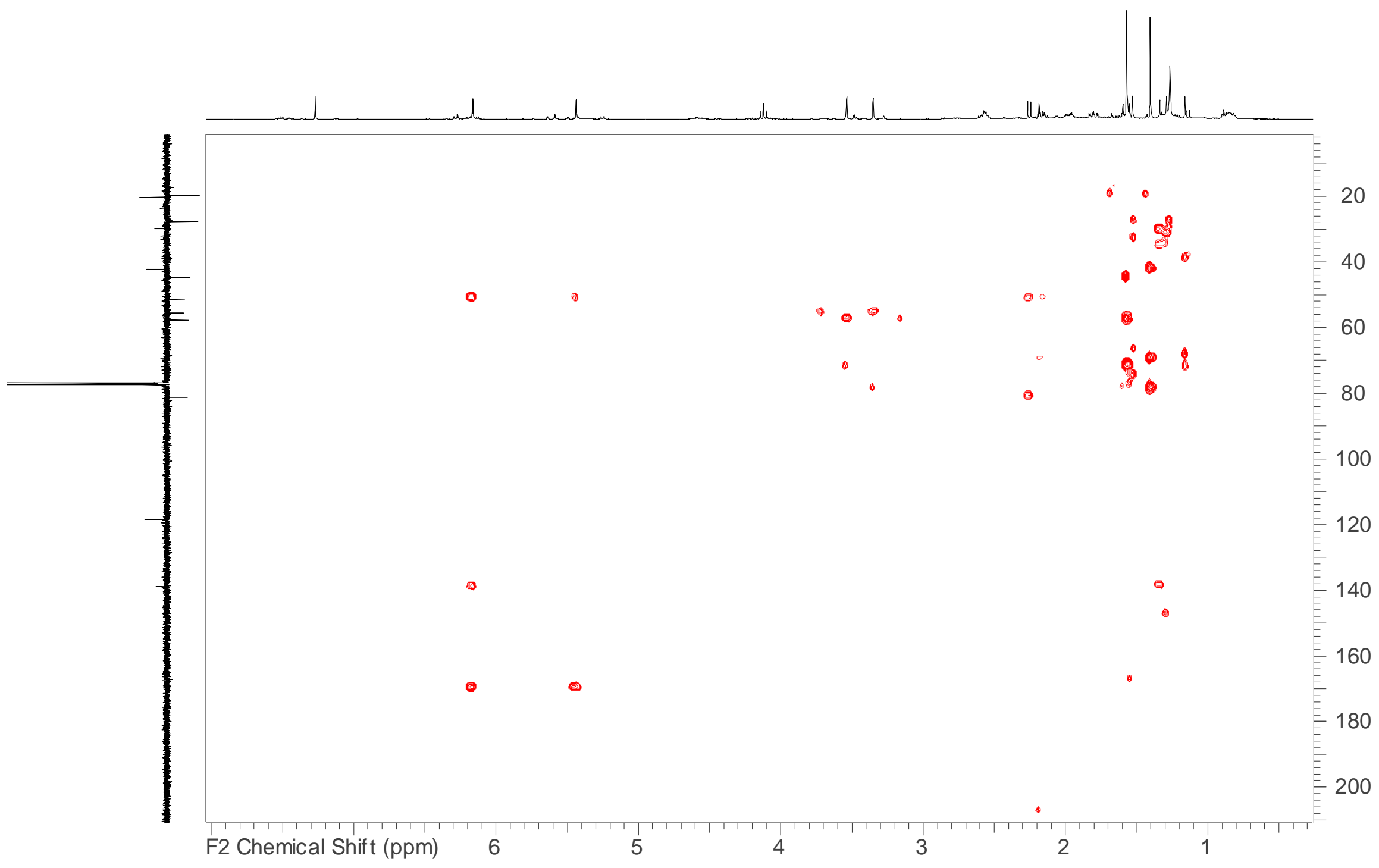

Figure S16. HMBC spectrum of compound $2\left(500 \mathrm{MHz}, \mathrm{CDCl}_{3}\right)$. 


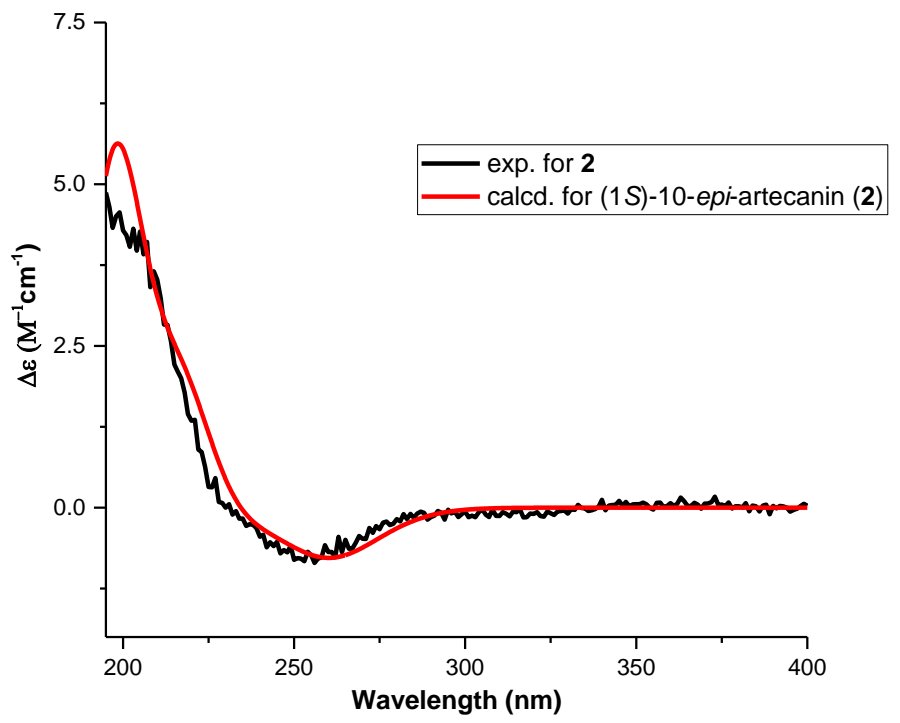

Figure S17. Comparison of experimental and computed ECD spectra for compound 2 in $\mathrm{MeOH}$. 

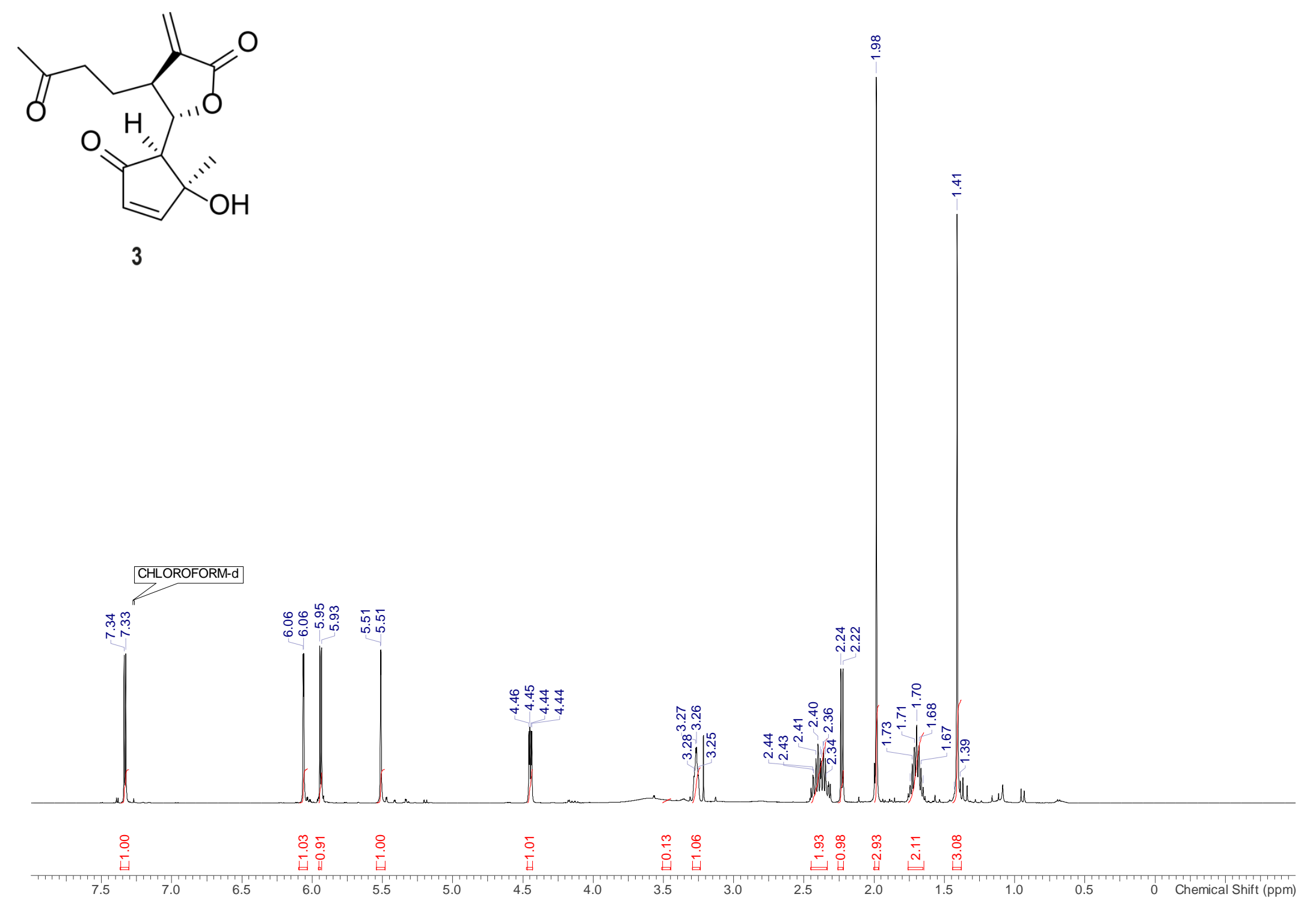

Figure S18. ${ }^{1} \mathrm{H}$ NMR spectrum of compound $3\left(500 \mathrm{MHz}, \mathrm{CDCl}_{3}\right)$. 


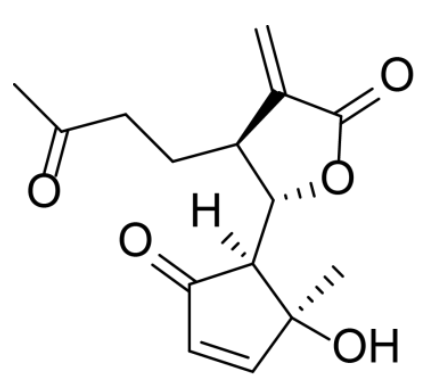

3

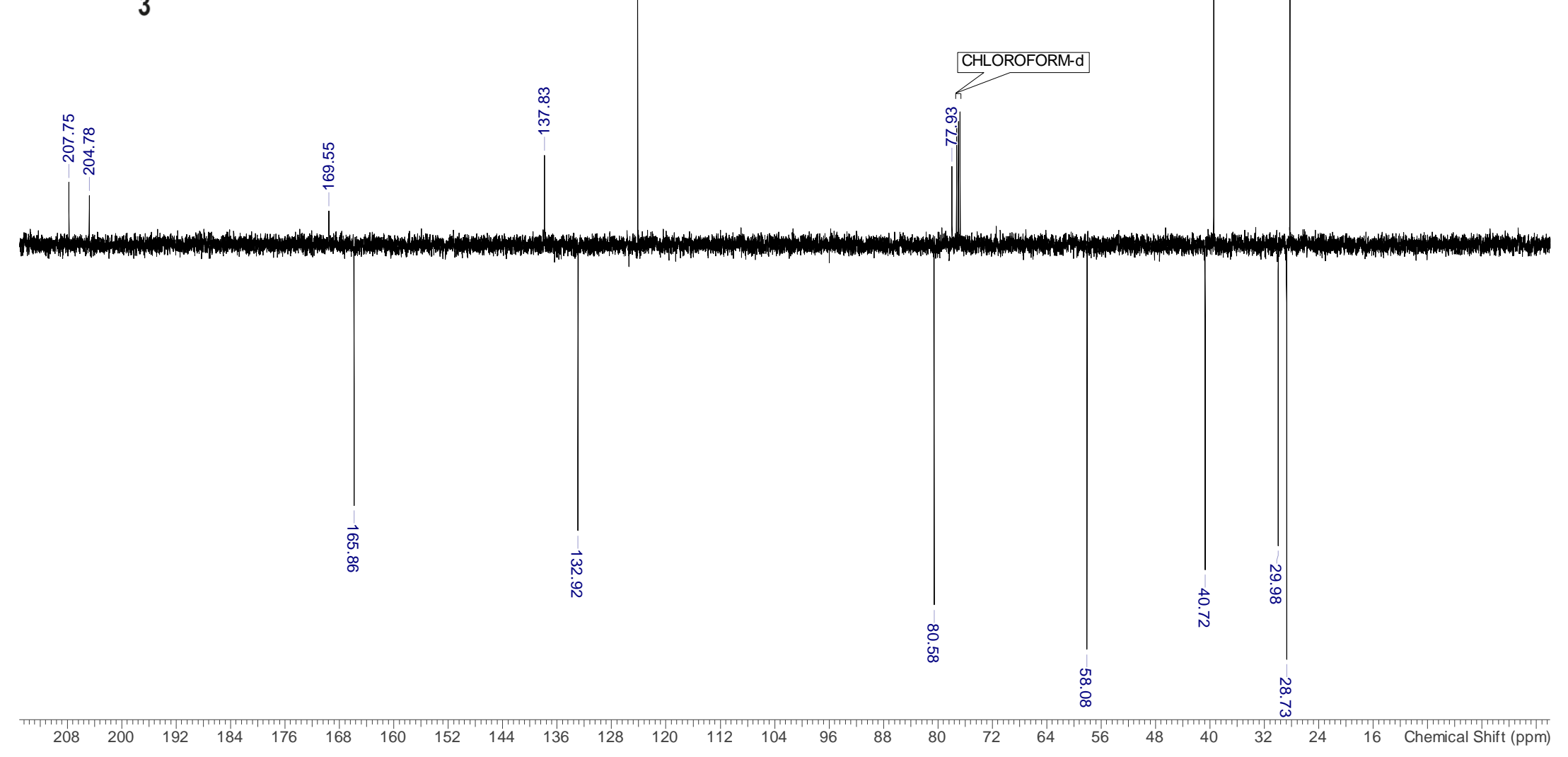

Figure S19. ${ }^{13} \mathrm{C}$-DEPTq spectrum of compound $3\left(125 \mathrm{MHz}, \mathrm{CDCl}_{3}\right)$. 


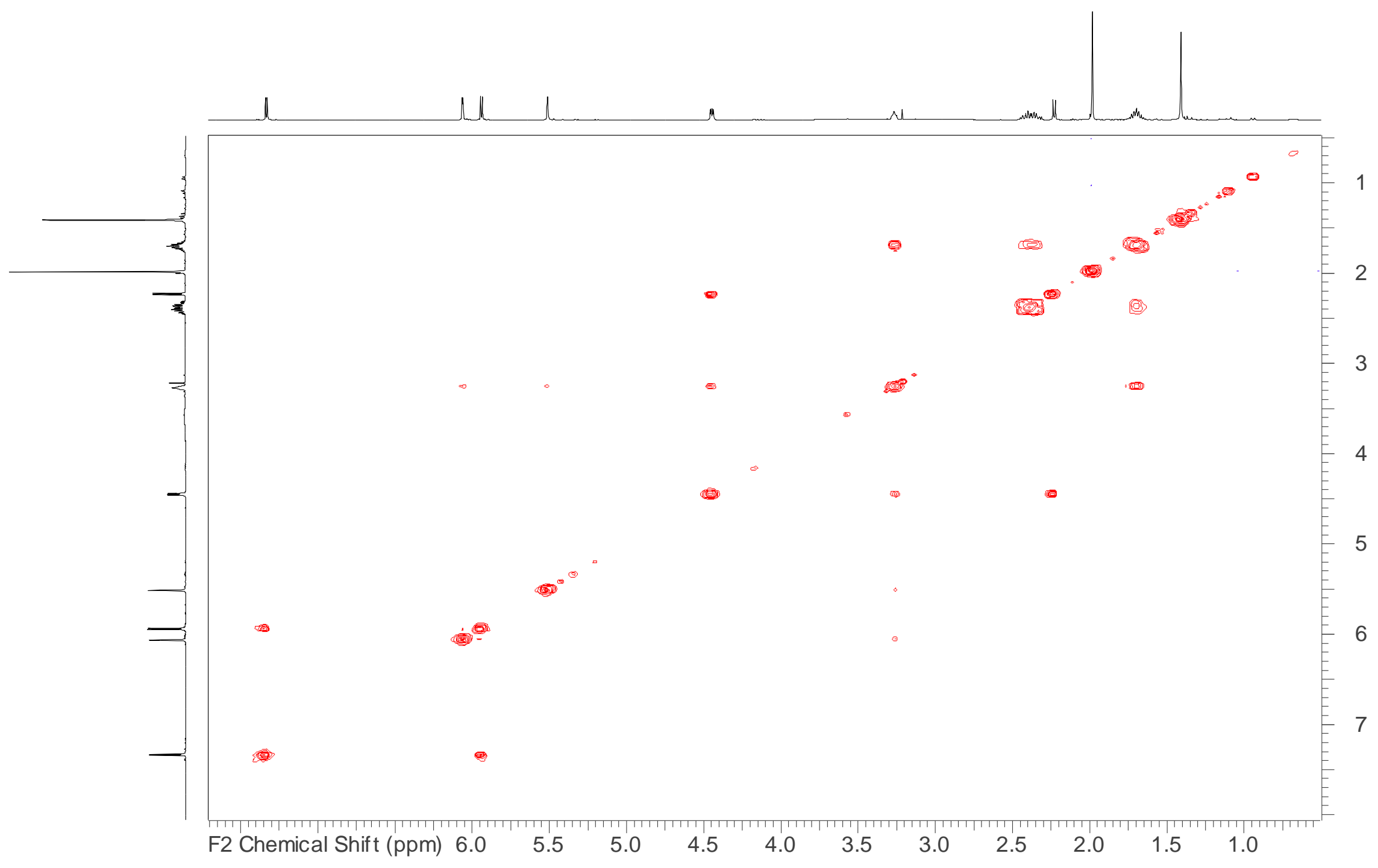

Figure S20. 1H 1H COSY spectrum of compound 3 (500 MHz, CDCl3). 


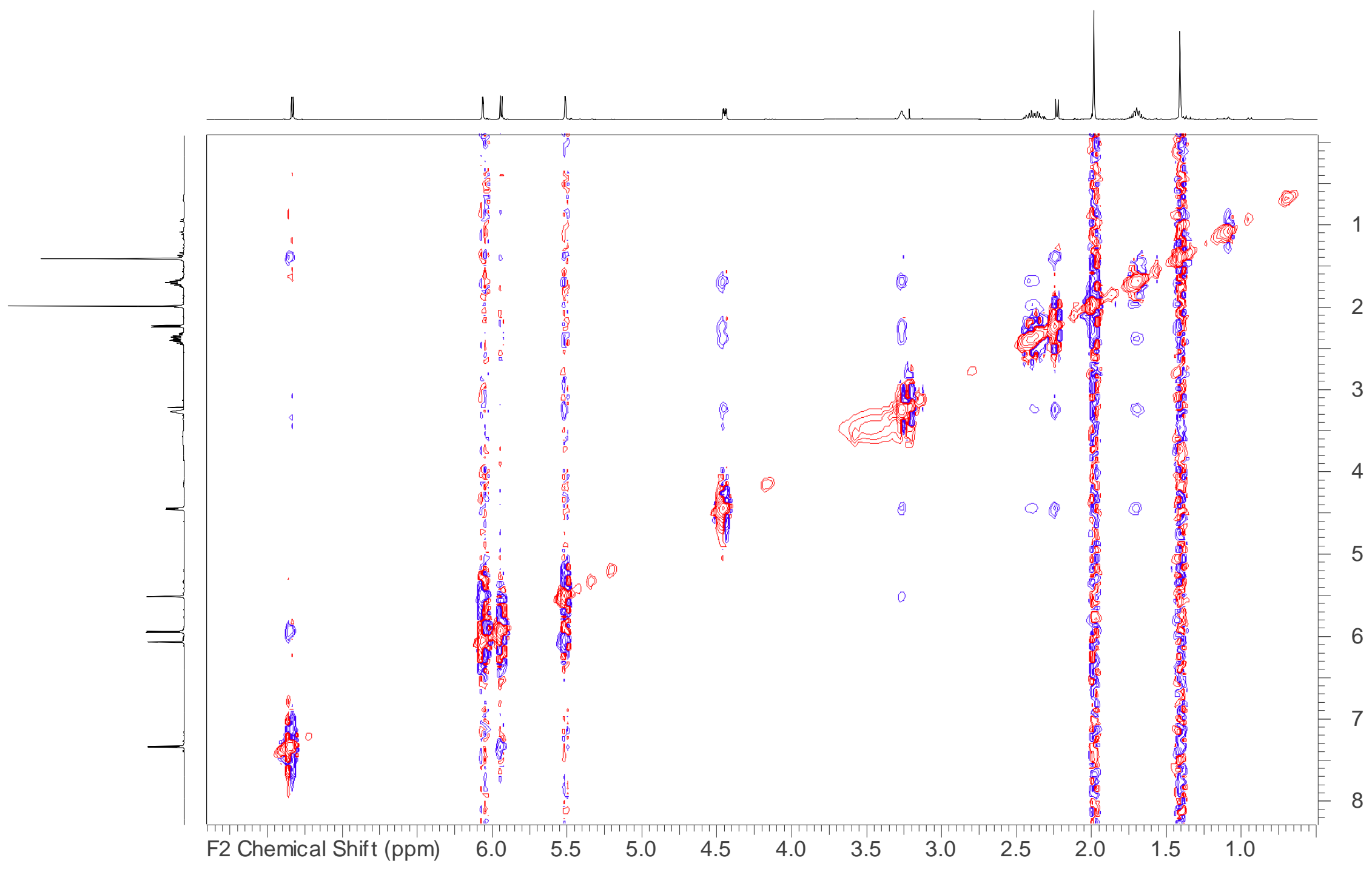

Figure S21. 1H 1H NOESY spectrum of compound 3 (500 MHz, CDCl3) 


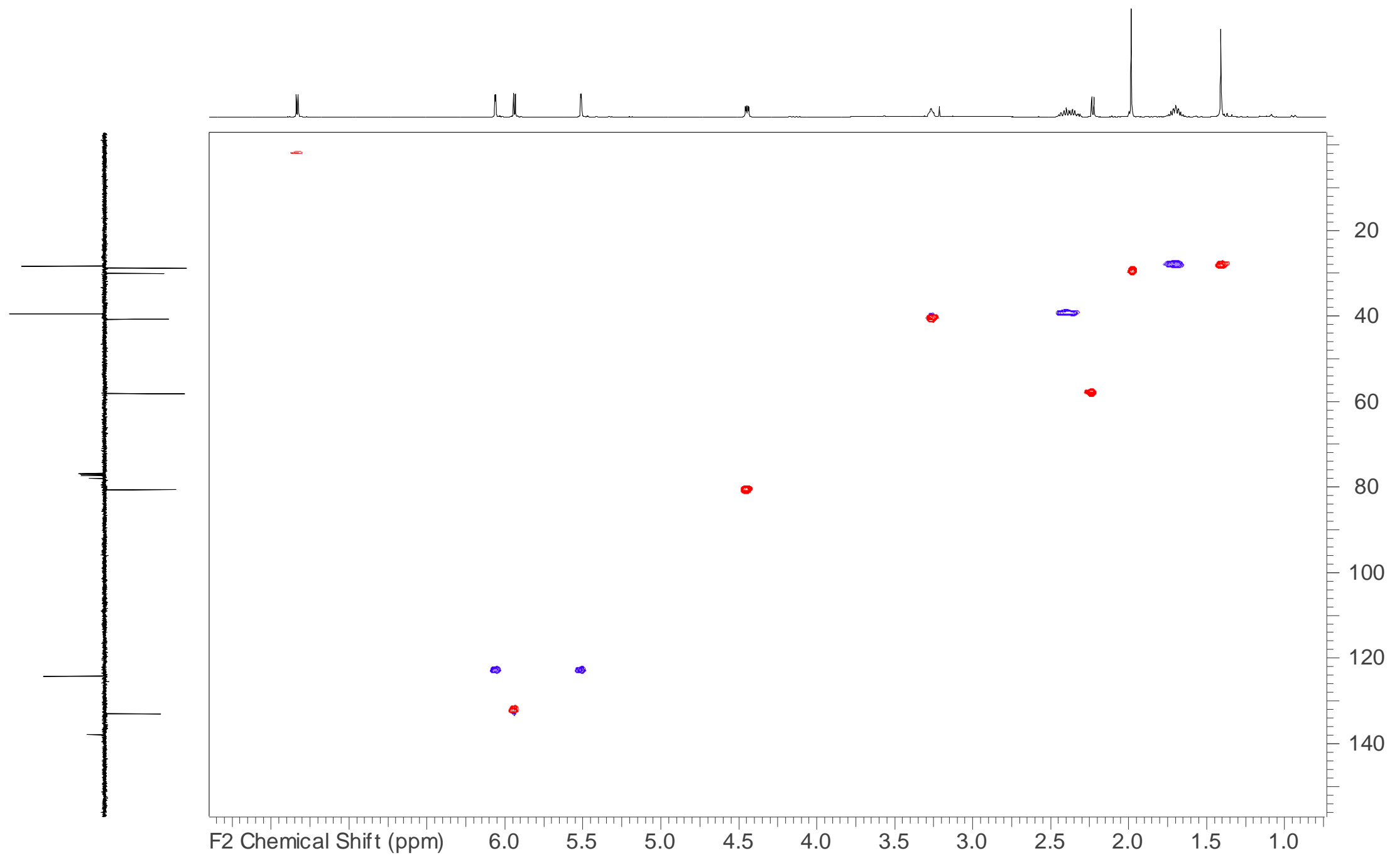

Figure S22. HSQC-DEPT spectrum of compound 3 (500 MHz, CDCl3). 


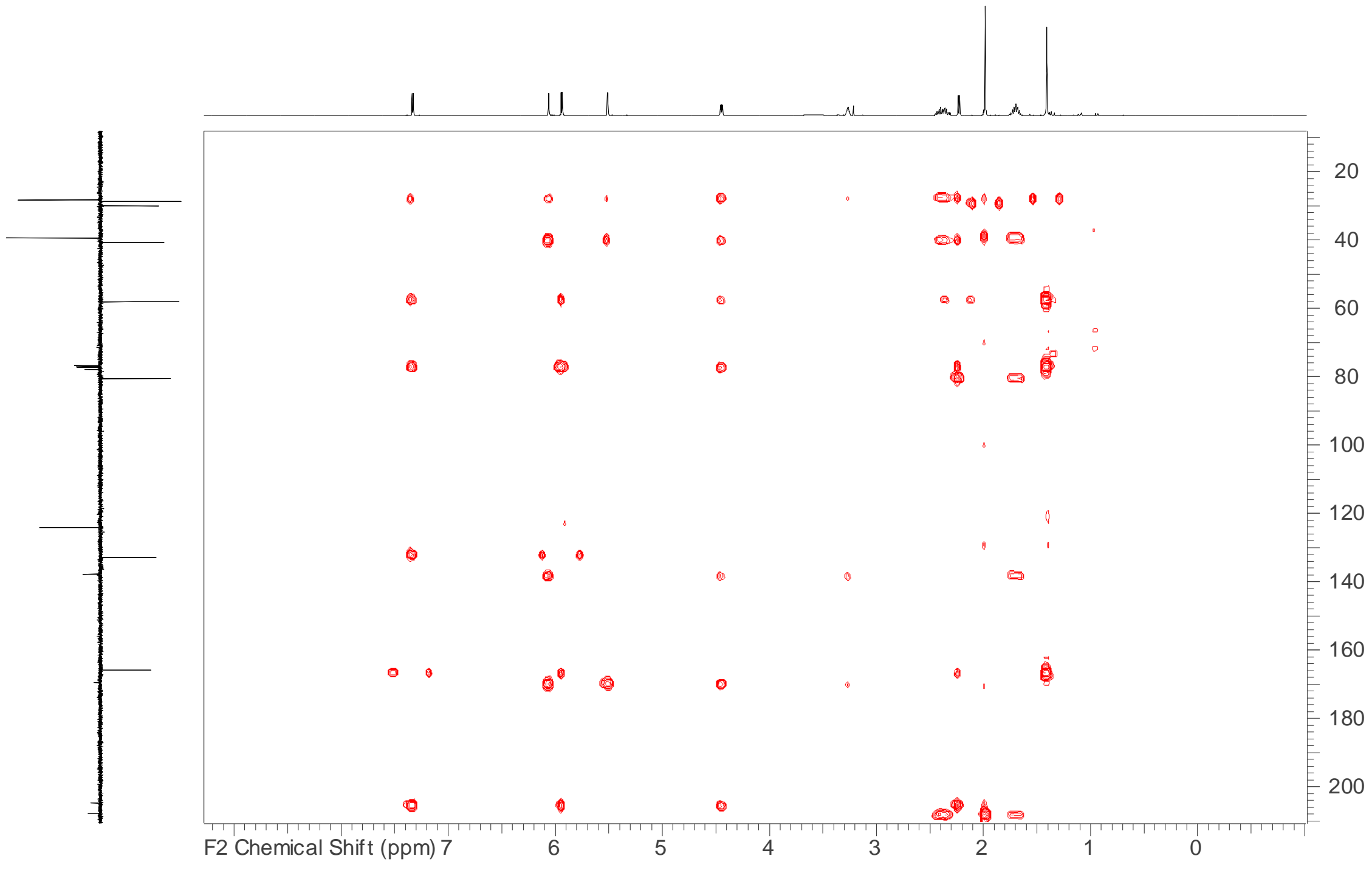

Figure S23. HMBC spectrum of compound 3 (500 MHz, CDCl3). 

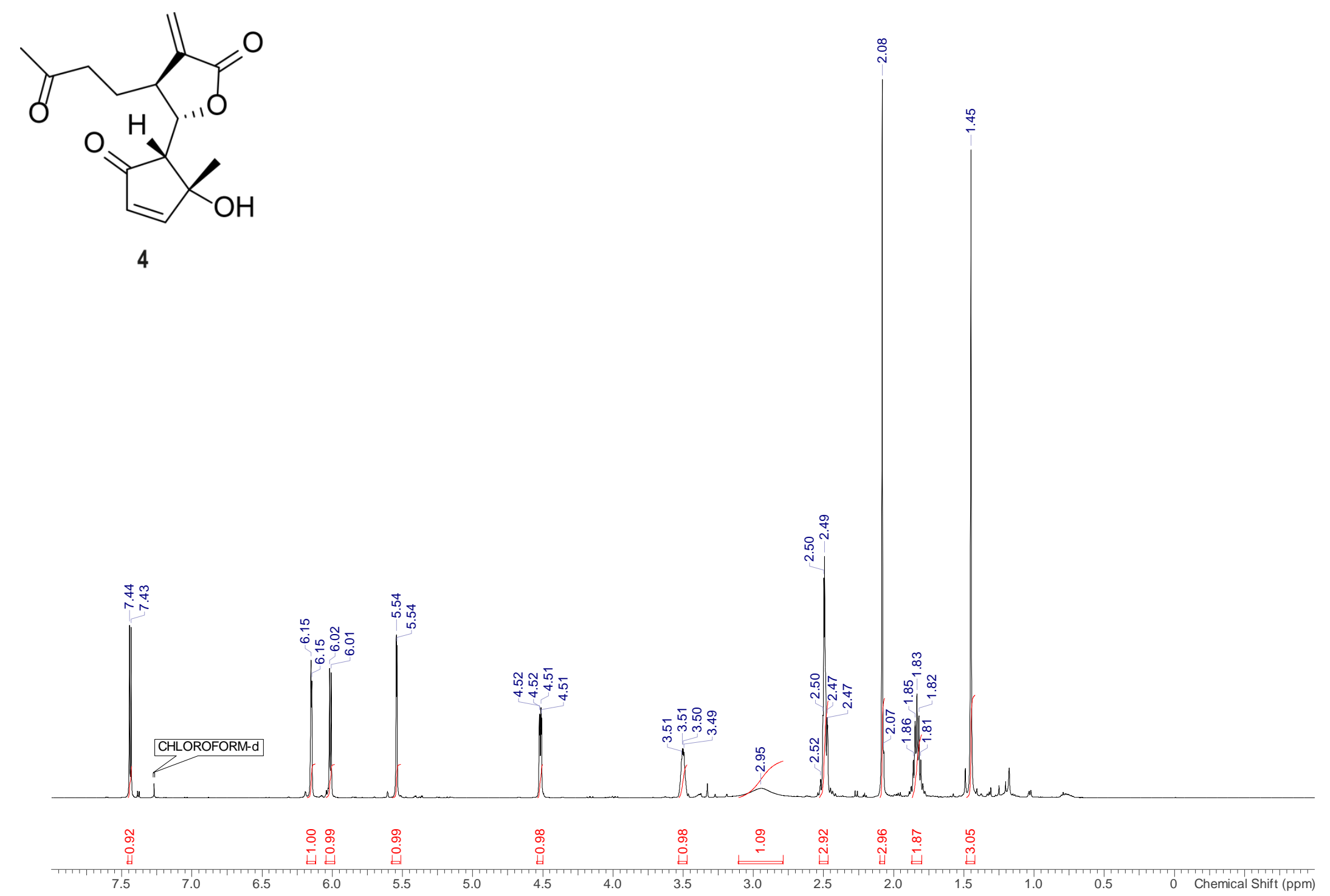

Figure S24. ${ }^{1} \mathrm{H}$ NMR spectrum of compound $4\left(500 \mathrm{MHz}, \mathrm{CDCl}_{3}\right)$. 


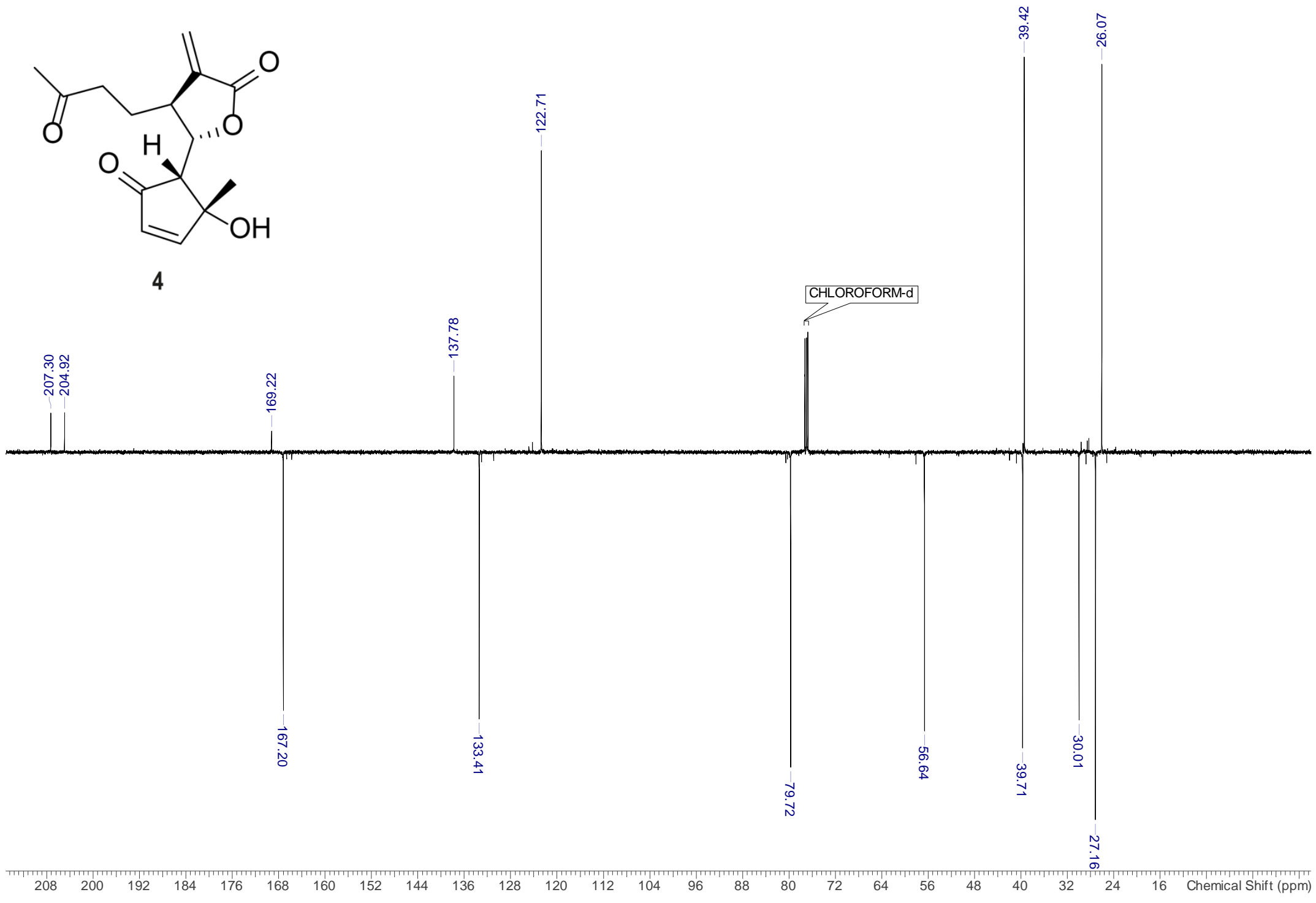

Figure S25. ${ }^{13} \mathrm{C}$-DEPTq spectrum of compound $4\left(125 \mathrm{MHz}, \mathrm{CDCl}_{3}\right)$. 


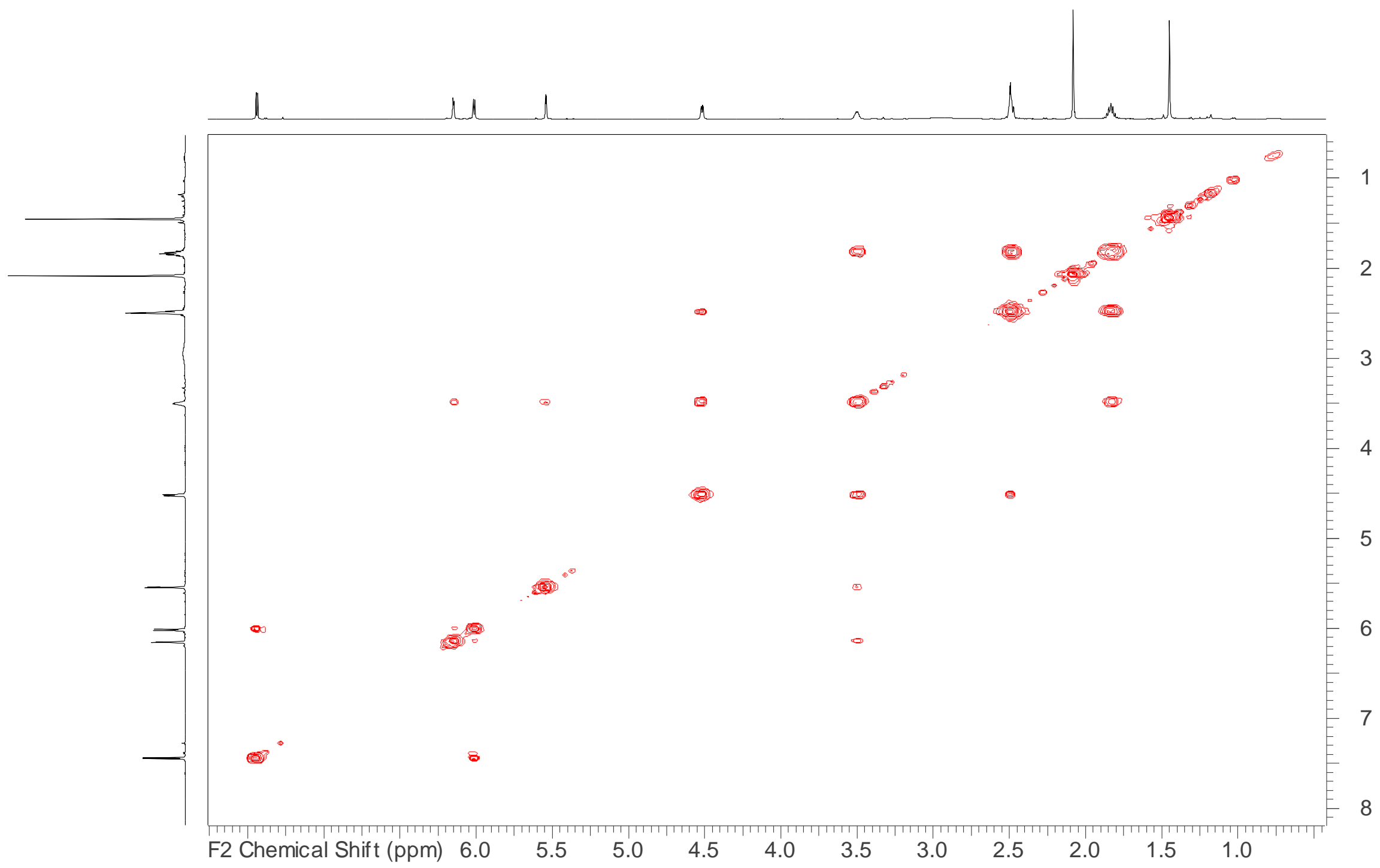

Figure S26. ${ }^{1} \mathrm{H}-{ }^{1} \mathrm{H}$ COSY spectrum of compound 4 (500 MHz, $\left.\mathrm{CDCl}_{3}\right)$. 


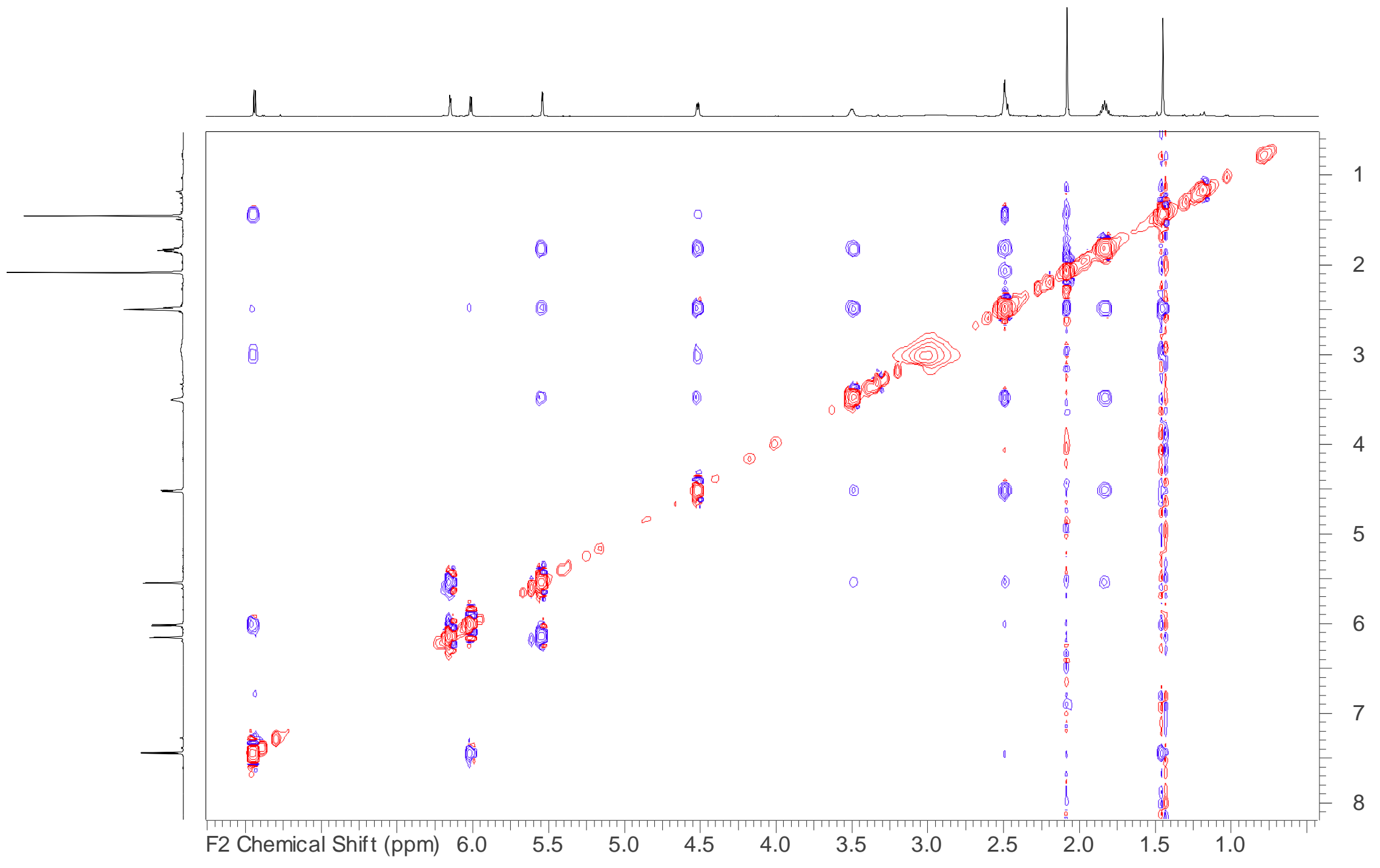

Figure S27. ${ }^{1} \mathrm{H}-{ }^{1} \mathrm{H}$ NOESY spectrum of compound $4\left(500 \mathrm{MHz}, \mathrm{CDCl}_{3}\right)$. 


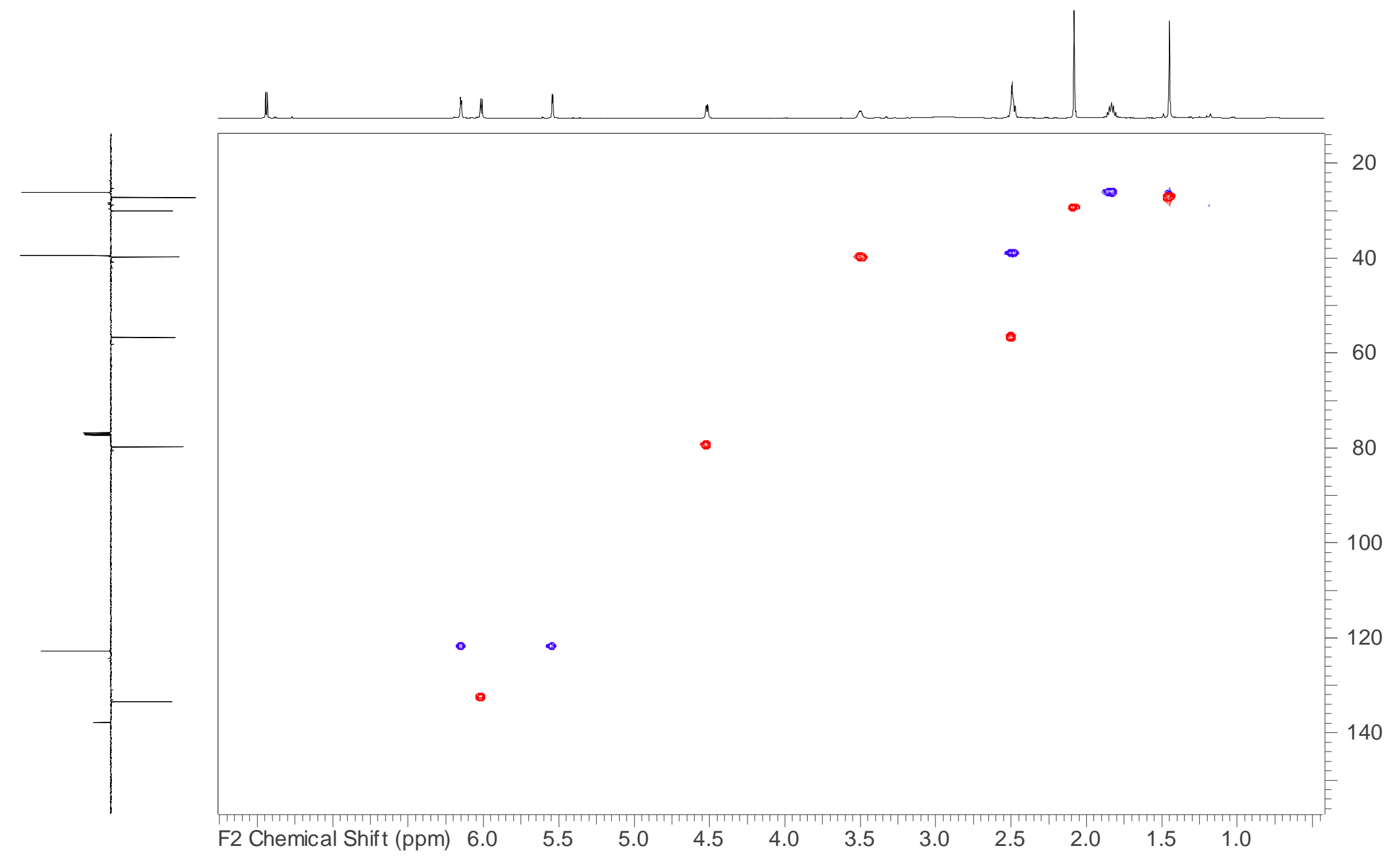

Figure S28. HSQC-DEPT spectrum of compound 4 (500 MHz, $\mathrm{CDCl}_{3}$ ). 


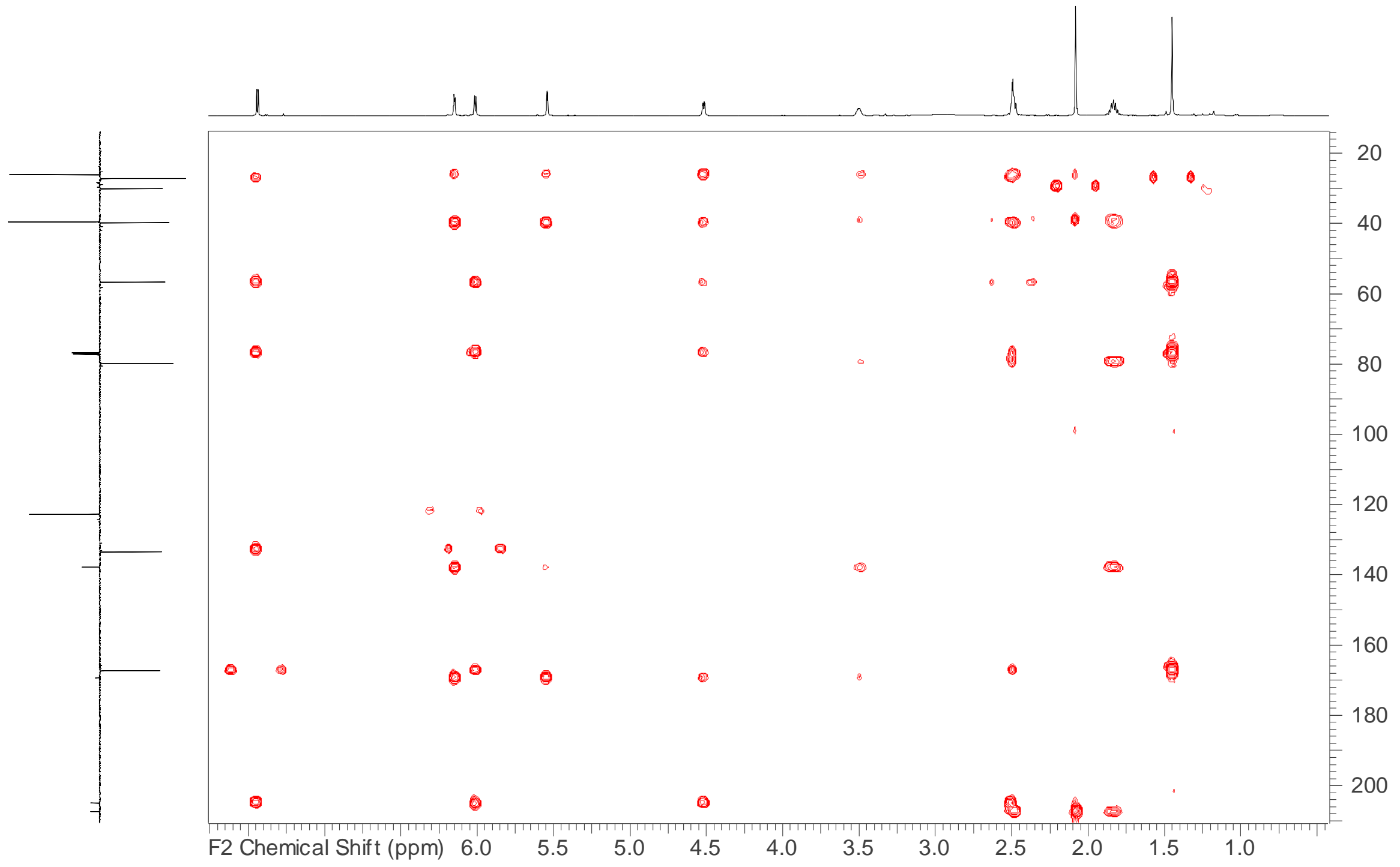

Figure S29. HMBC spectrum of compound $4\left(500 \mathrm{MHz}, \mathrm{CDCl}_{3}\right)$. 


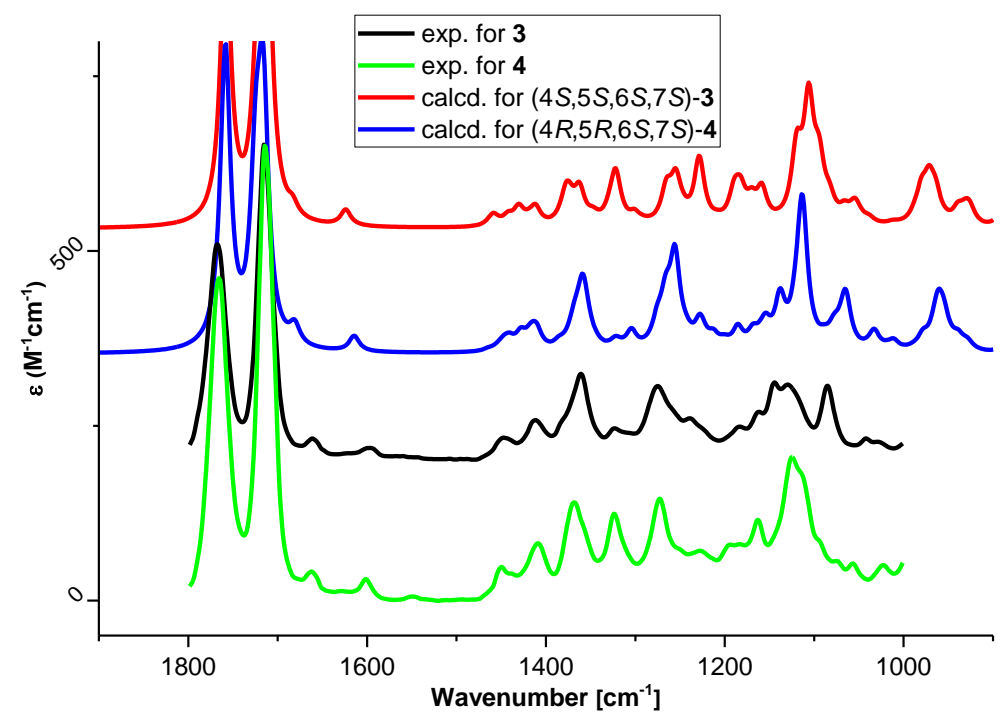

Figure S30. Comparison of experimental and computed IR spectra in chloroform for compounds 3 and 4 in the region 1900-1050 $\mathrm{cm}^{-1}$. Computed spectra (compounds 3 and 4) were scaled according to the overall maximal SimVCD value (0.9795). 

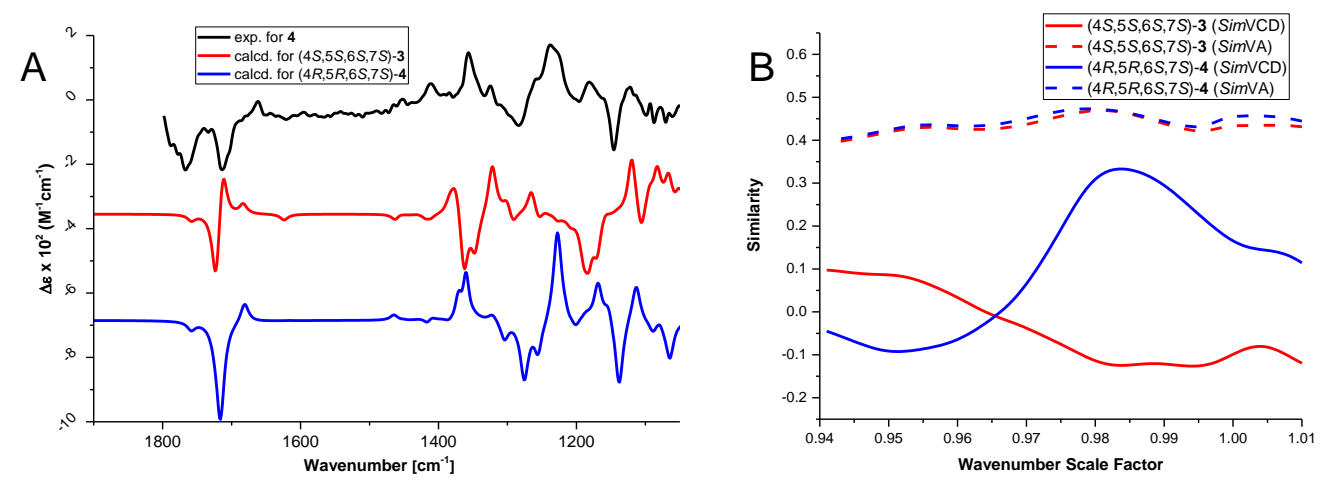

Figure S31. Comparison of experimental and computed VCD spectra in chloroform for compound 4. The region of $1900-1050 \mathrm{~cm}^{-1}$ is shown (A). Similarities (SimVA and SimVCD) of the experimental VA and VCD spectra of $\mathbf{4}$ to the calculated spectra of possible stereoisomers were plotted as functions of wavenumber scale factor (B). The wavenumber scale factor corresponding to the maximal Sim VCD value in B (0.9795) was used to scale the computed spectra in A. 

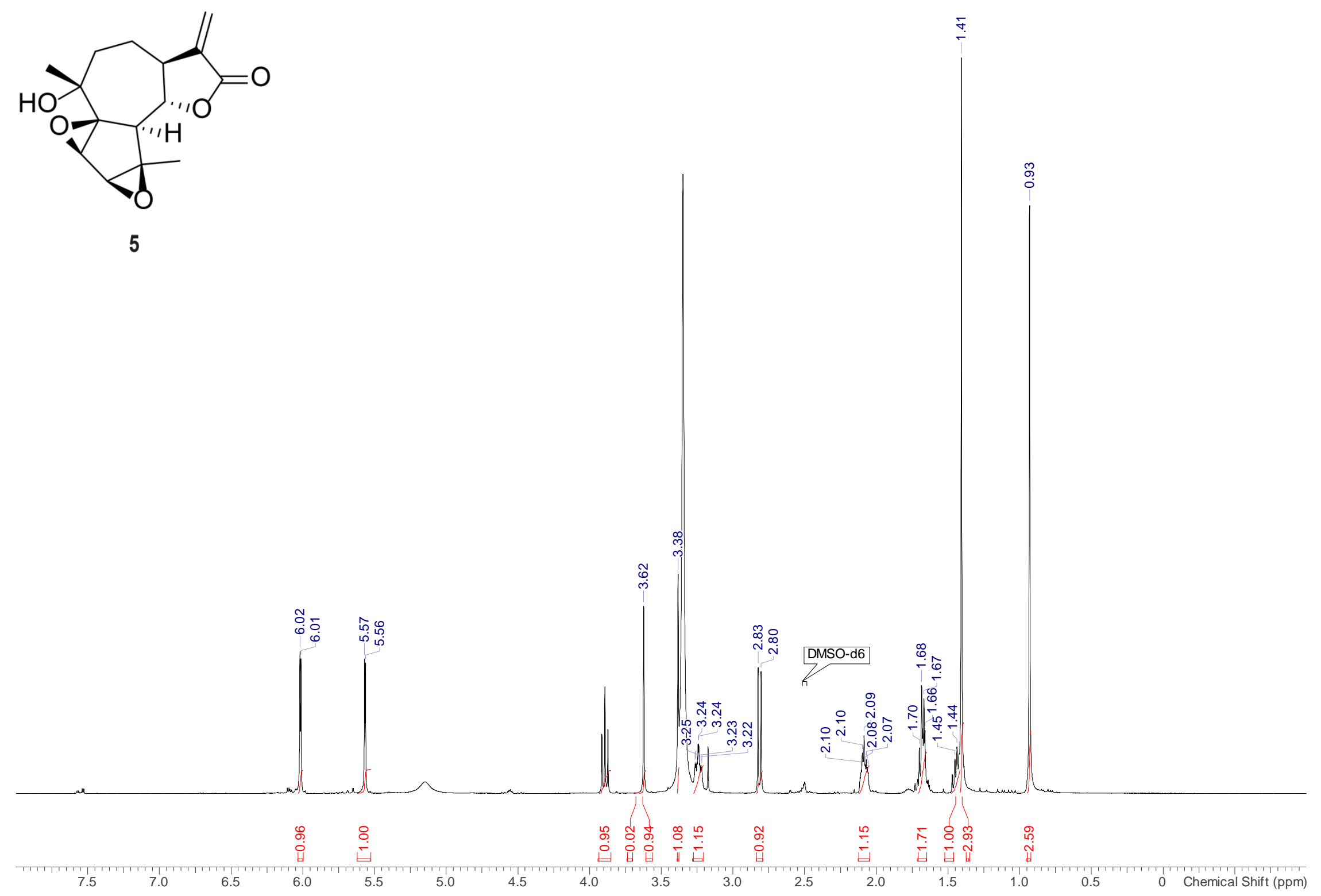

Figure S32. ${ }^{1} \mathrm{H}$ NMR spectrum of compound 5 (500 MHz, DMSO). 


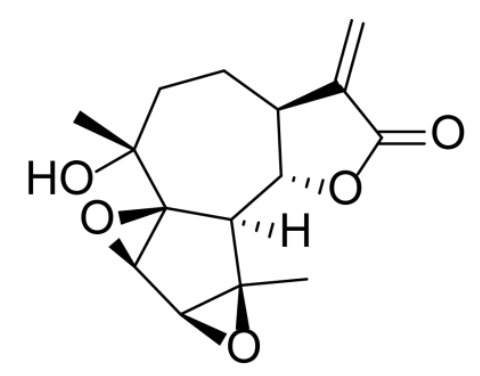

5

DMSO-d6

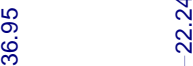

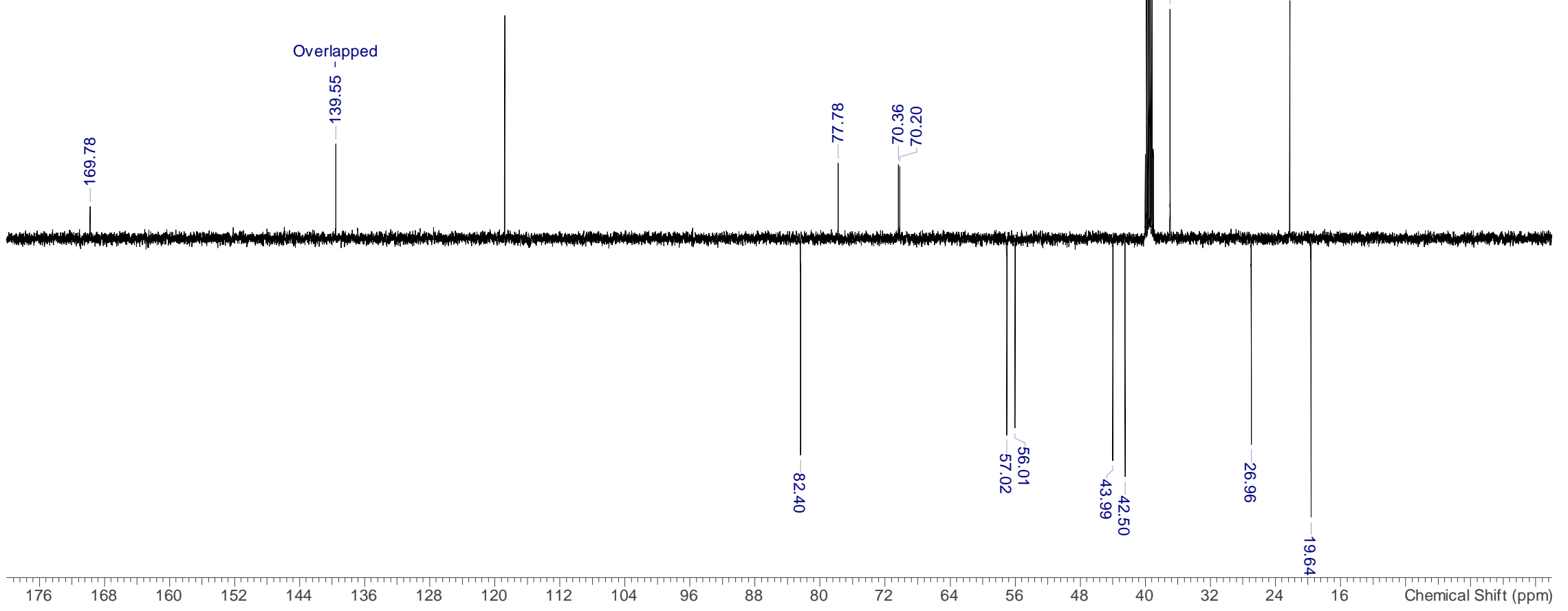

Figure S33. ${ }^{13} \mathrm{C}-\mathrm{DEPT}$ q spectrum of compound 5 (125 MHz, DMSO). 


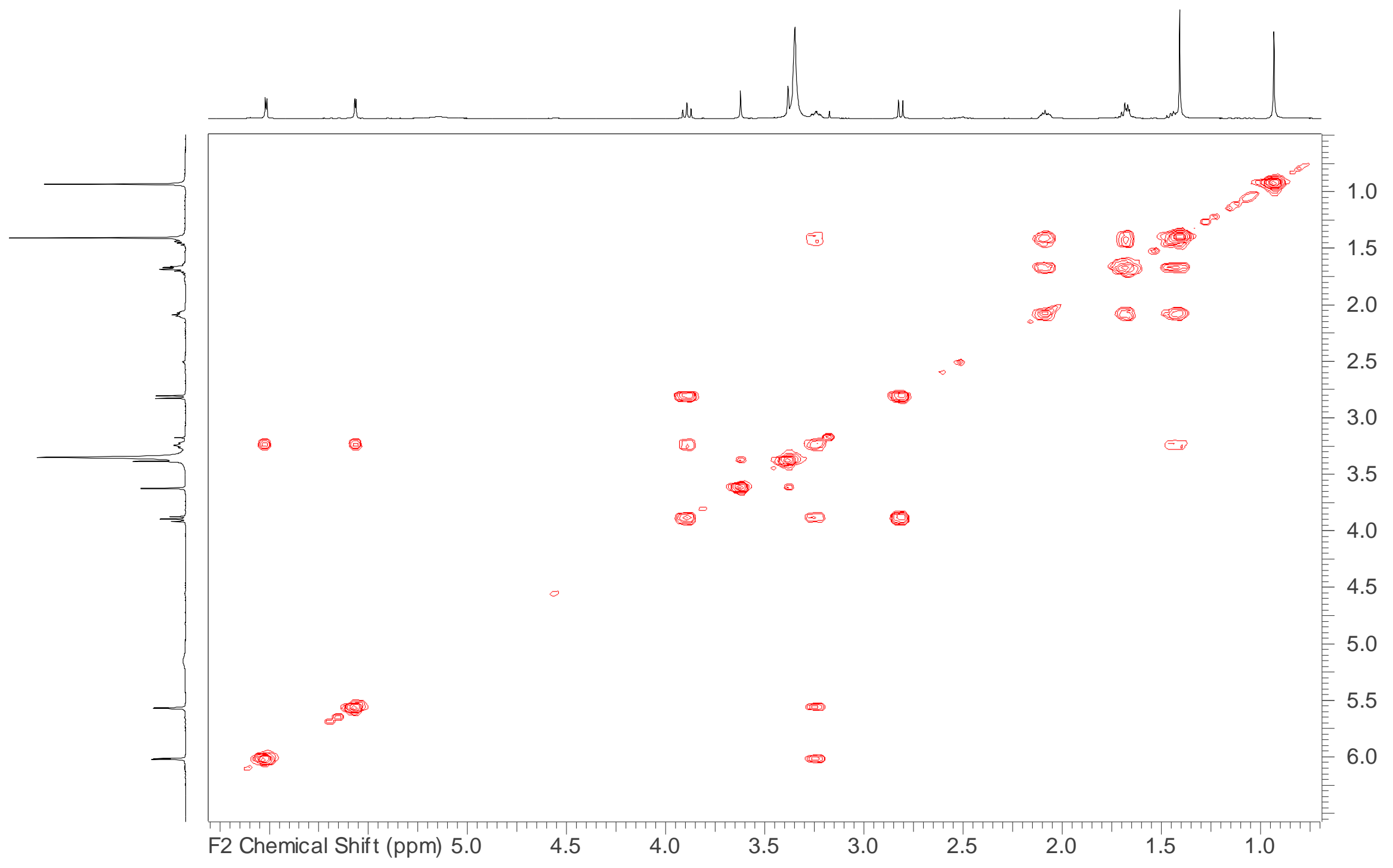

Figure S34. ${ }^{1} \mathrm{H}-{ }^{1} \mathrm{H}$ COSY spectrum of compound 5 (500 MHz, DMSO). 


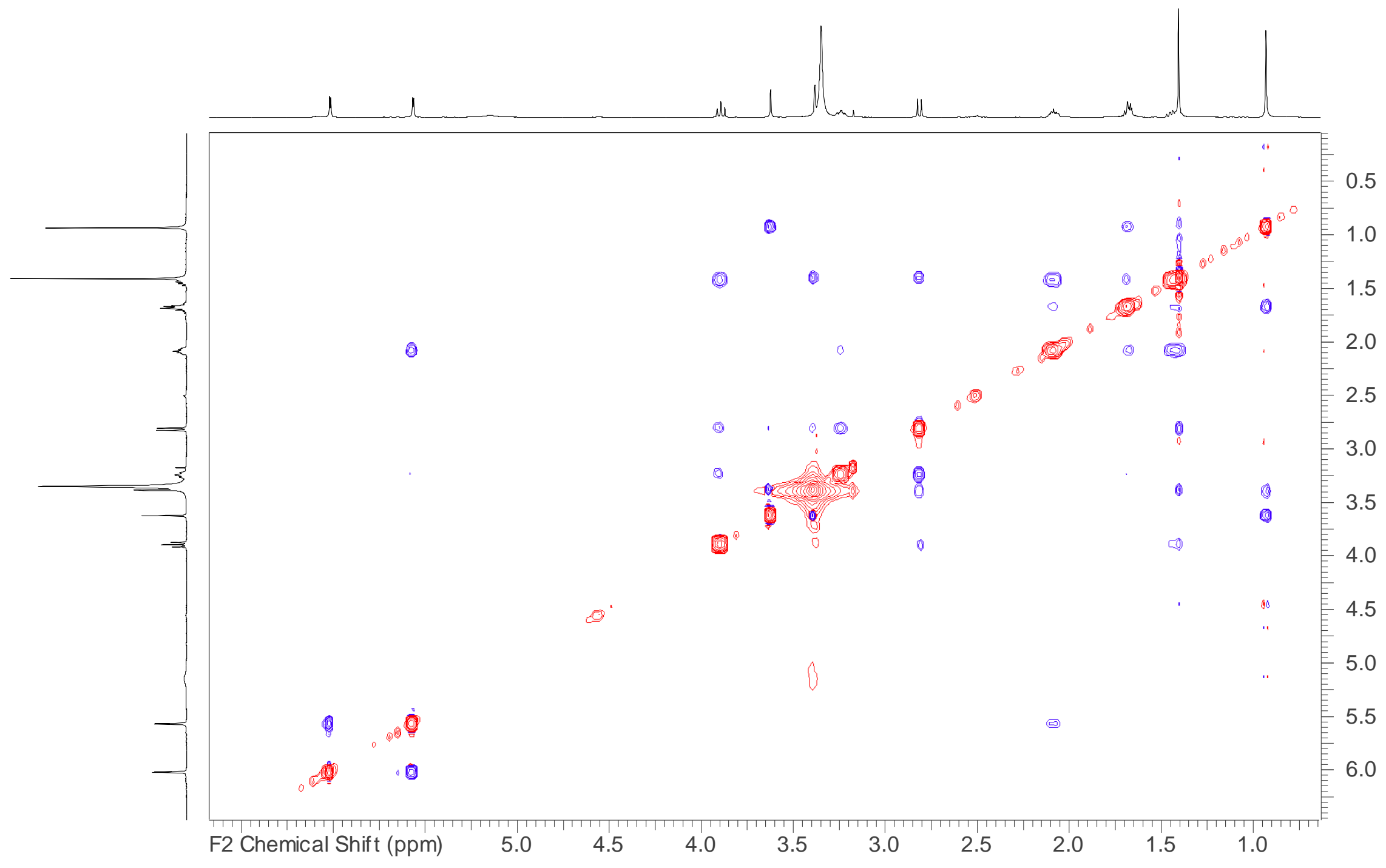

Figure S35. ${ }^{1} \mathrm{H}-{ }^{1} \mathrm{H}$ NOESY spectrum of compound 5 (500 MHz, DMSO). 


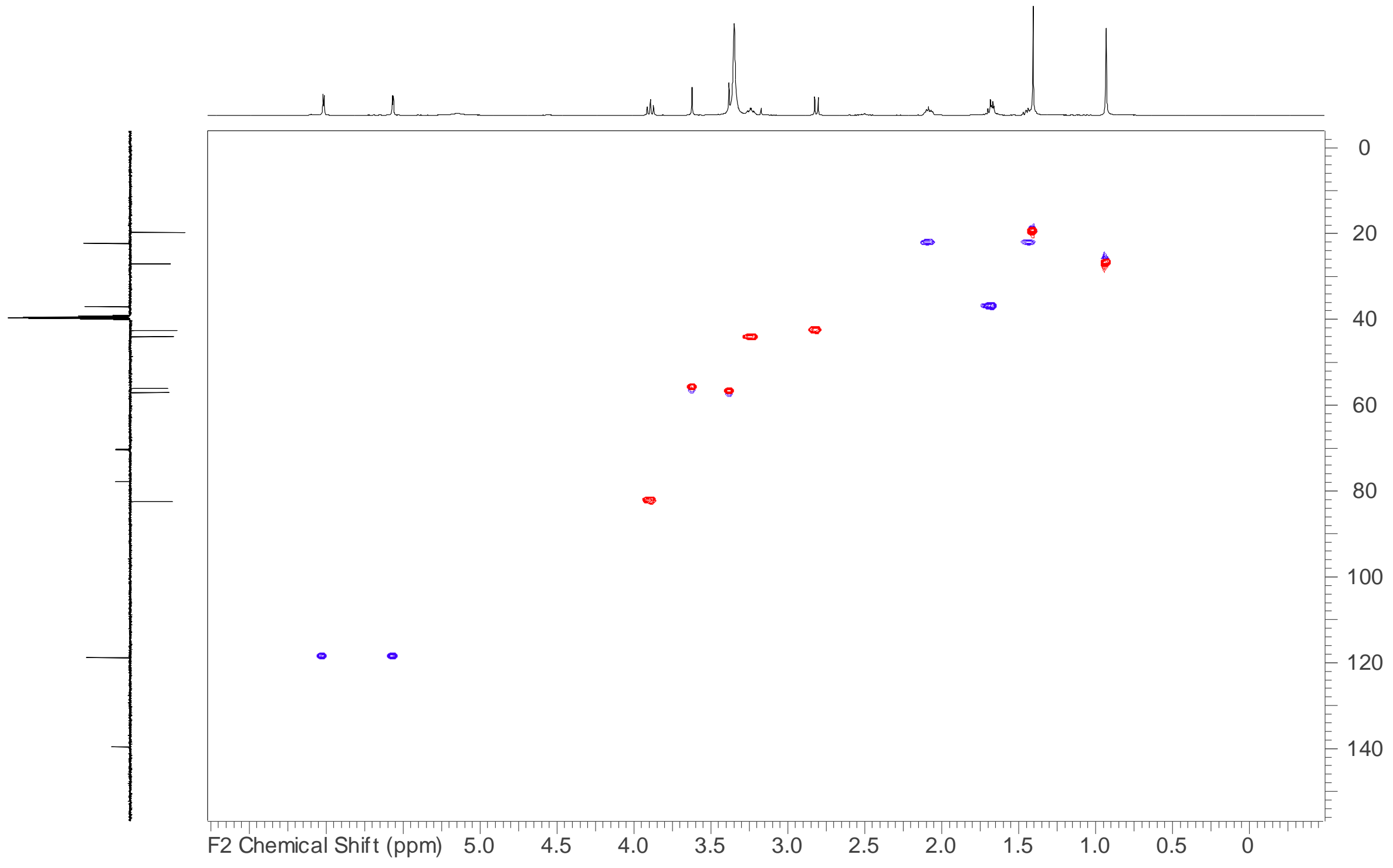

Figure S36. HSQC-DEPT spectrum of compound 5 (500 MHz, DMSO). 


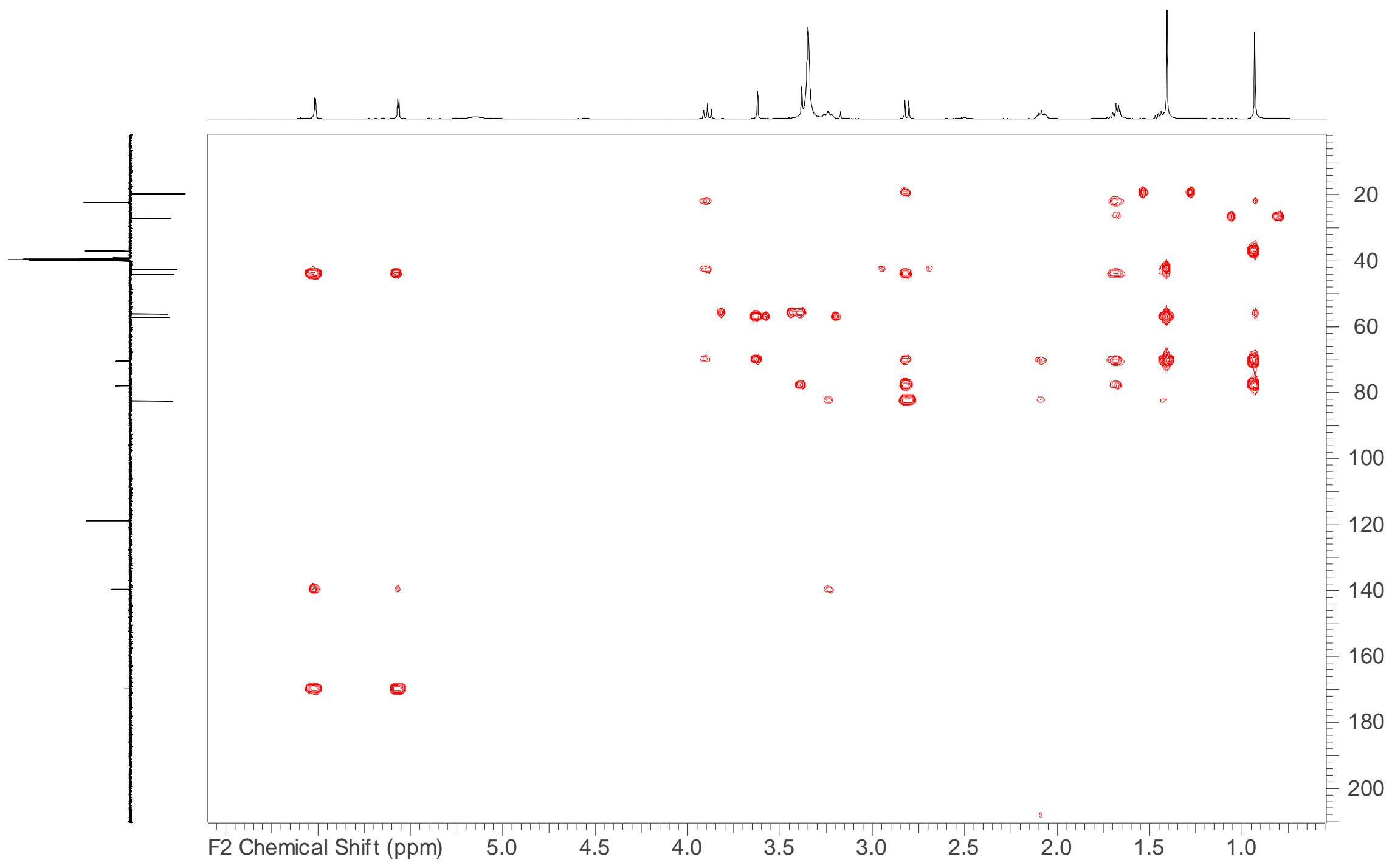

Figure S37. HMBC spectrum of compound 5 (500 MHz, DMSO). 


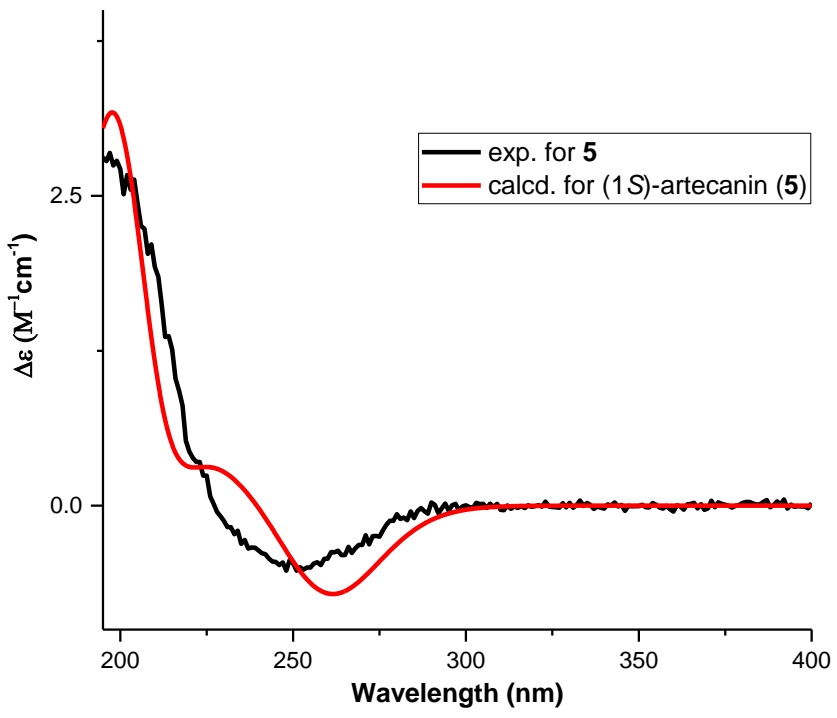

Figure S38. Comparison of experimental and computed ECD spectra for compound $5 \mathrm{in} \mathrm{MeOH}$. 


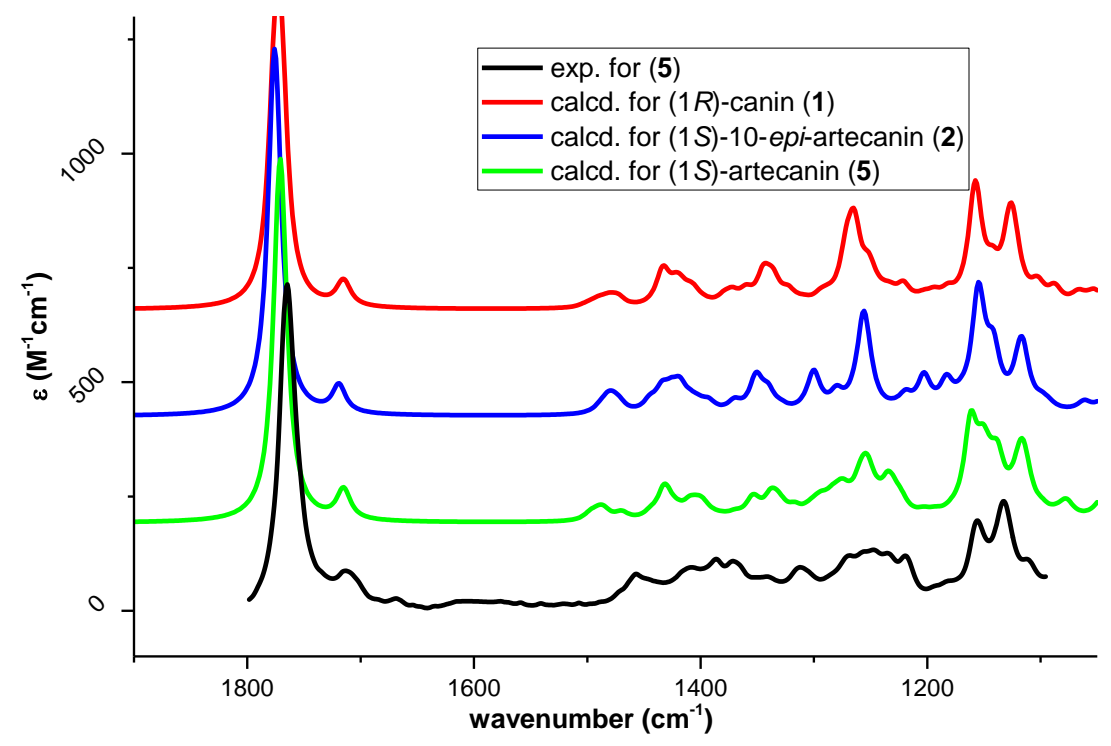

Figure S39. Comparison of experimental and computed IR spectra in DMSO-d 6 for compound 5 in the region 1900-1050 $\mathrm{cm}^{-1}$. Computed spectra (compounds $\mathbf{1}, \mathbf{2}$ and 5) were scaled according to the overall maximal $\operatorname{Sim} \mathrm{VCD}$ value (0.9955). 

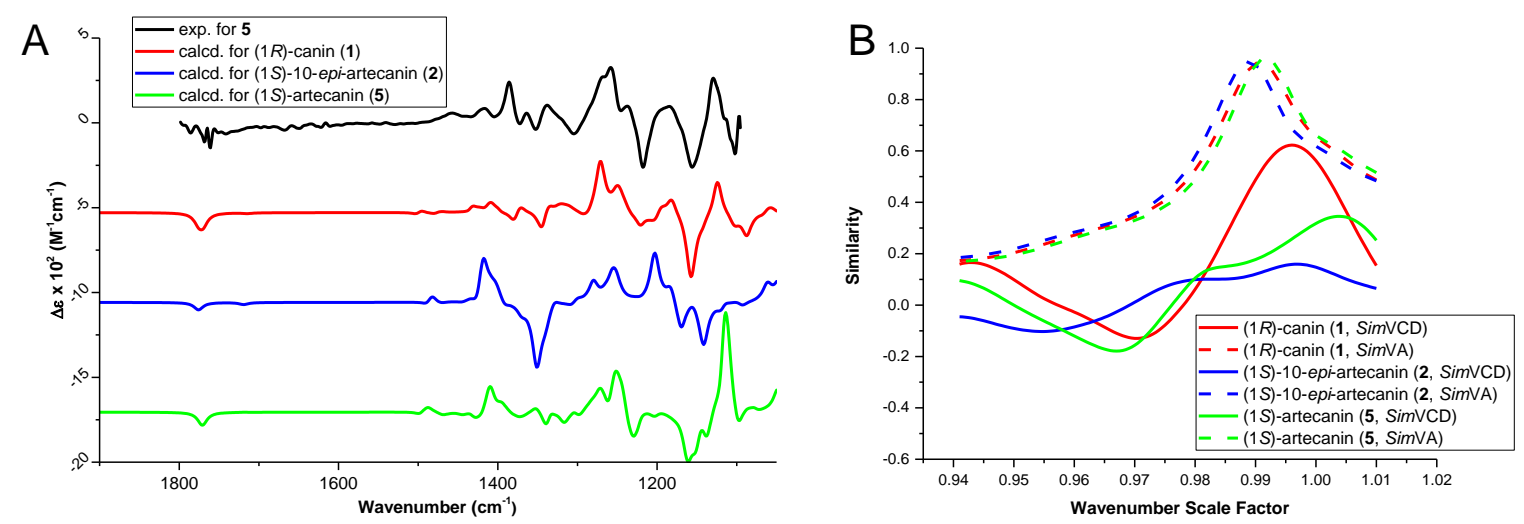

Figure S40. Comparison of experimental and computed VCD spectra in DMSO-d 6 for compound 5. The region of $1900-1050 \mathrm{~cm}^{-1}$ is shown (A). Similarities ( $\operatorname{Sim}$ VA and $\operatorname{Sim}$ VCD) of the experimental VA and VCD spectra of $\mathbf{5}$ to the calculated spectra of possible stereoisomers were plotted as functions of wavenumber scale factor (B). The wavenumber scale factor corresponding to the maximal Sim VCD value in B (0.9955) was used to scale the computed spectra in A. 


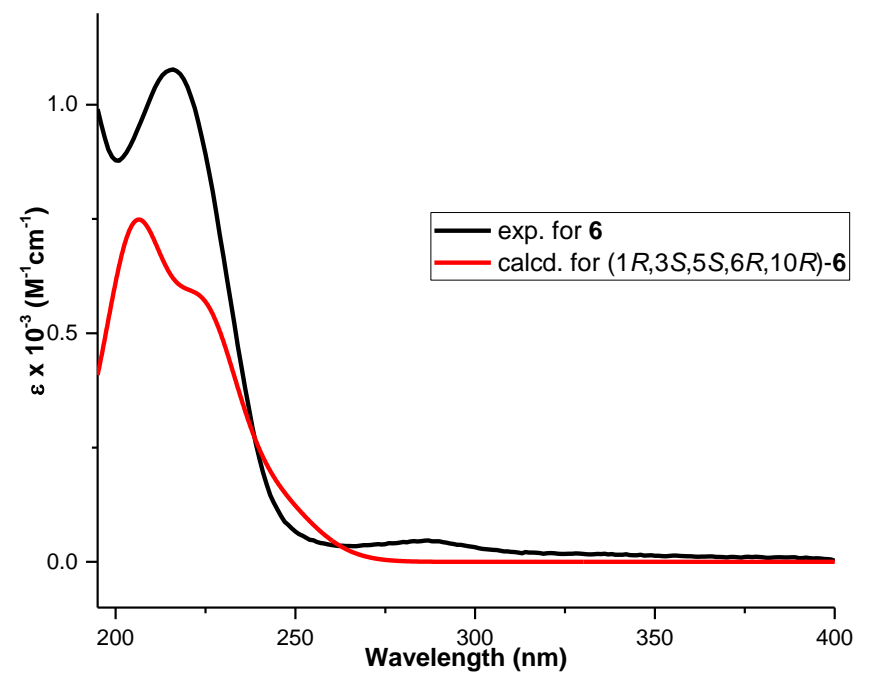

Figure S41. Comparison of experimental and computed UV spectra for compound 6 in $\mathrm{MeOH}$. 


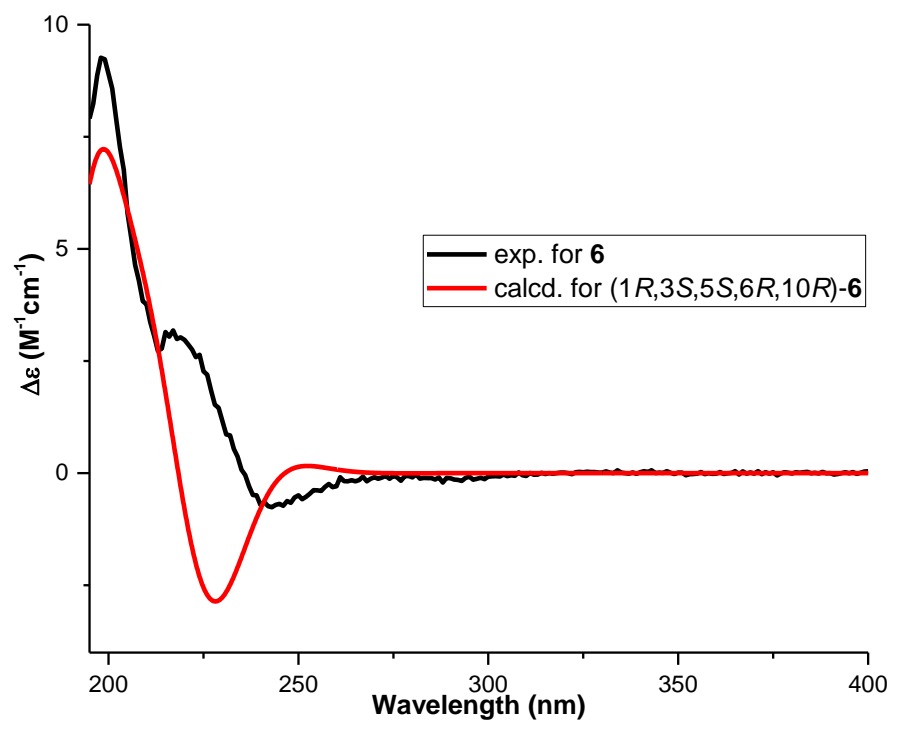

Figure S42. Comparison of experimental and computed ECD spectra for compound 6 in $\mathrm{MeOH}$. 


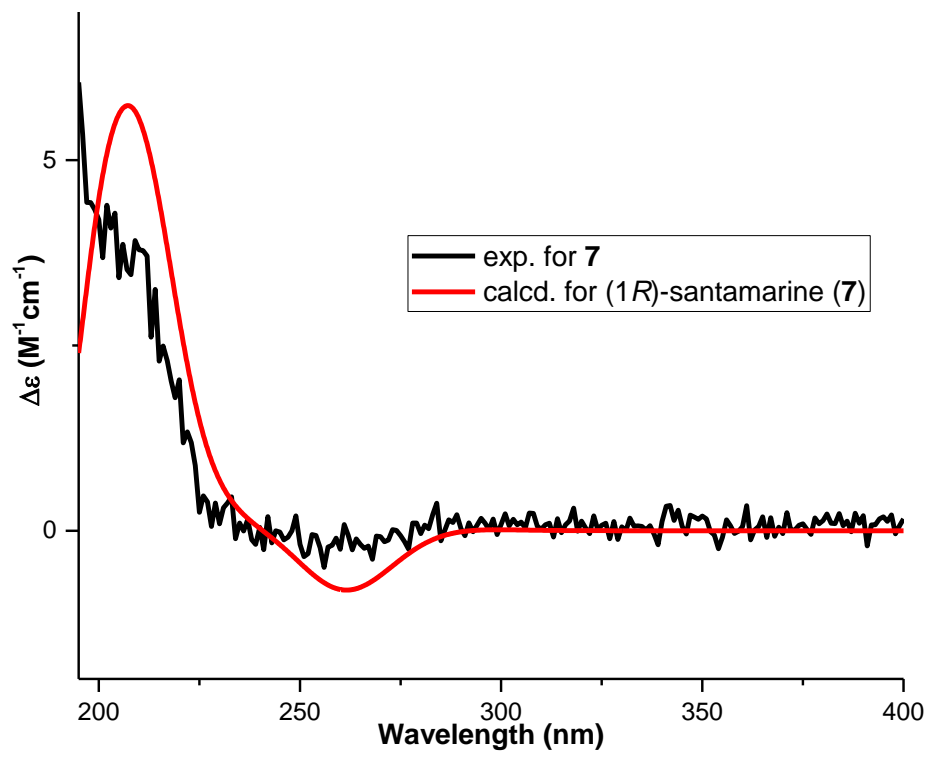

Figure S43. Comparison of experimental and computed ECD spectra for compound $7 \mathrm{in} \mathrm{MeOH}$. 
<smiles>CCC(C)C(=O)O[C@H]1C[C@H](O)[C@]2(C)CCC3=C(COC(C)=O)C(=O)O[C@@H]3[C@H]2[C@]1(C)O</smiles>

8
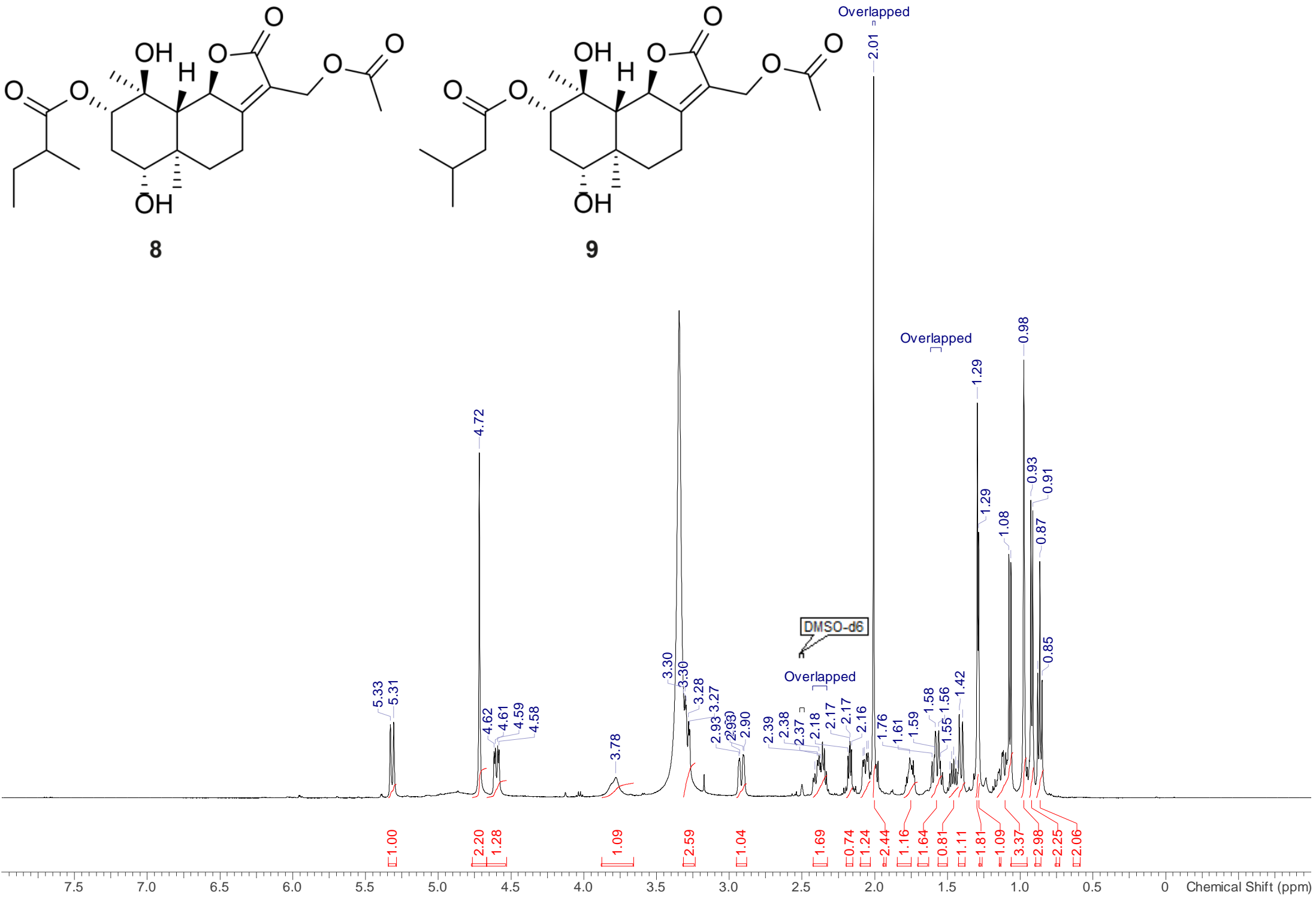

Figure S44. ${ }^{1} \mathrm{H}$ NMR spectrum of compounds 8 and 9 (500 MHz, DMSO). 
<smiles>CCC(C)C(=O)O[C@H]1C[C@H](O)[C@]2(C)CCC3=C(COC(C)=O)C(=O)O[C@@H]3[C@]2(C)[C@]1(C)O</smiles>

8<smiles>CC(=O)OCC1=C2CC[C@@]3([C@@H]4OC(=O)C(COC(C)=O)=C4COC(C)=O)[C@@H](O)C[C@H](OC(=O)CC(C)C)[C@@](C)(O)[C@H]3[C@@H]2OC1=O</smiles>

9
DMSO-d6

mo

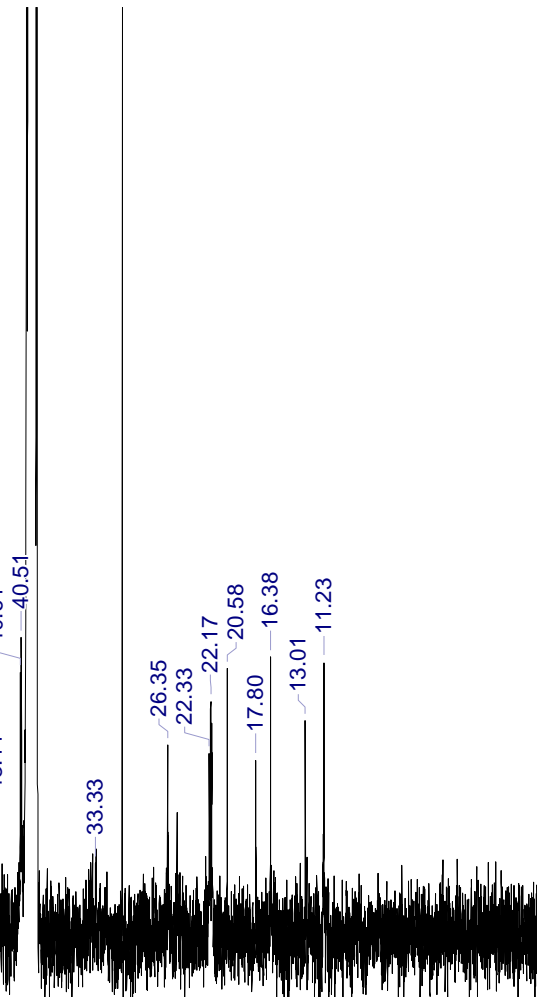

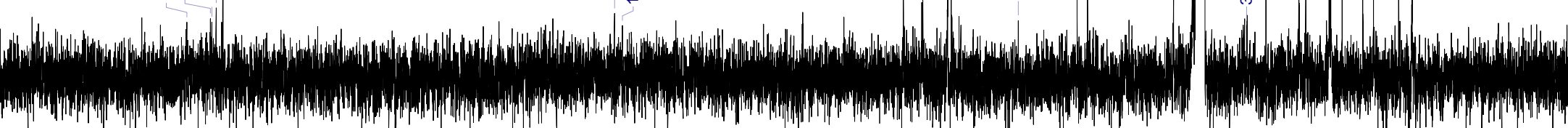

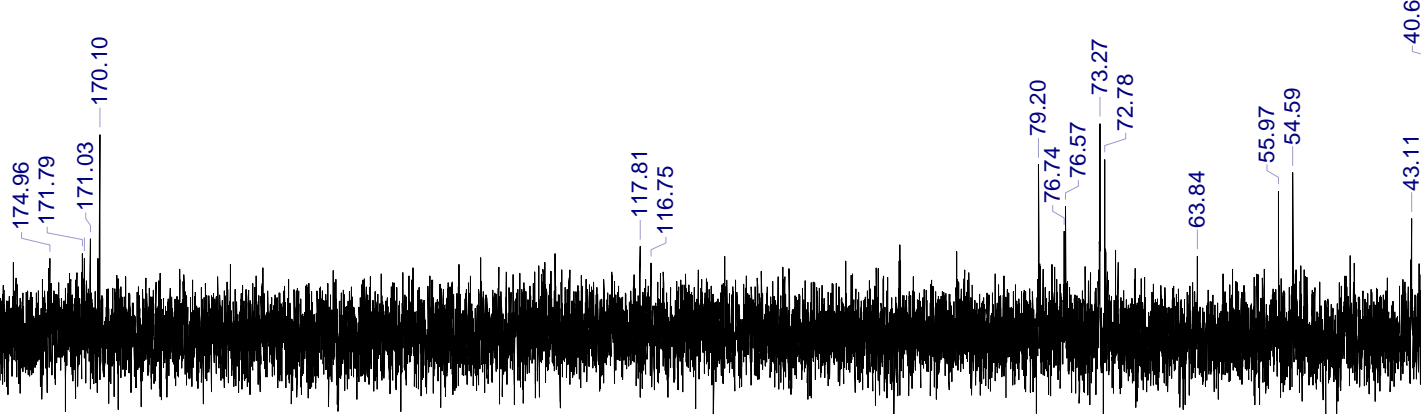

Figure S45. ${ }^{13} \mathrm{C}$ NMR spectrum of compounds 8 and $9\left(125 \mathrm{MHz}, \mathrm{CDCl}_{3}\right)$. 


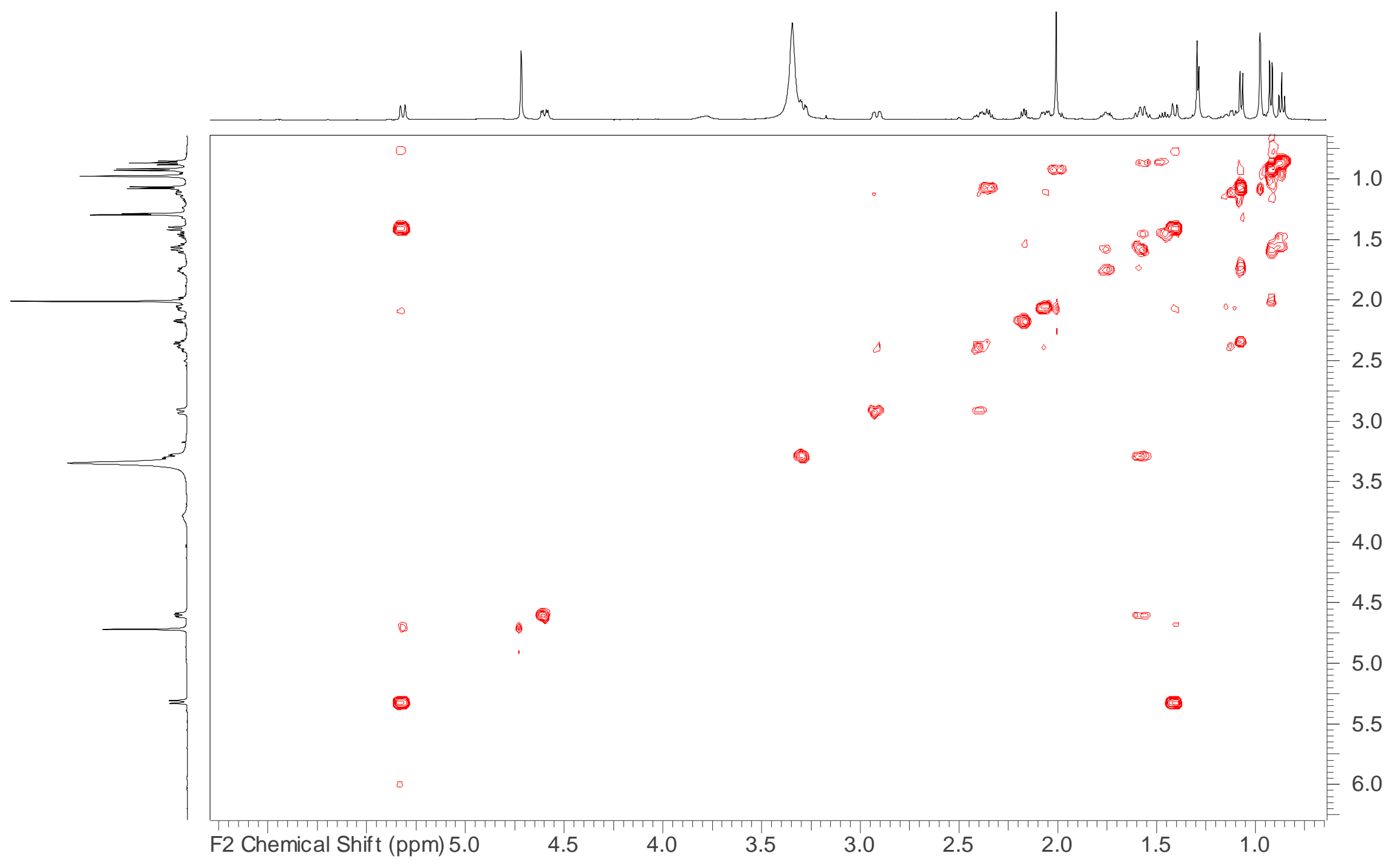

Figure S46. ${ }^{1} \mathrm{H}-{ }^{1} \mathrm{H}$ COSY spectrum of compounds 8 and $9\left(500 \mathrm{MHz}, \mathrm{CDCl}_{3}\right)$. 


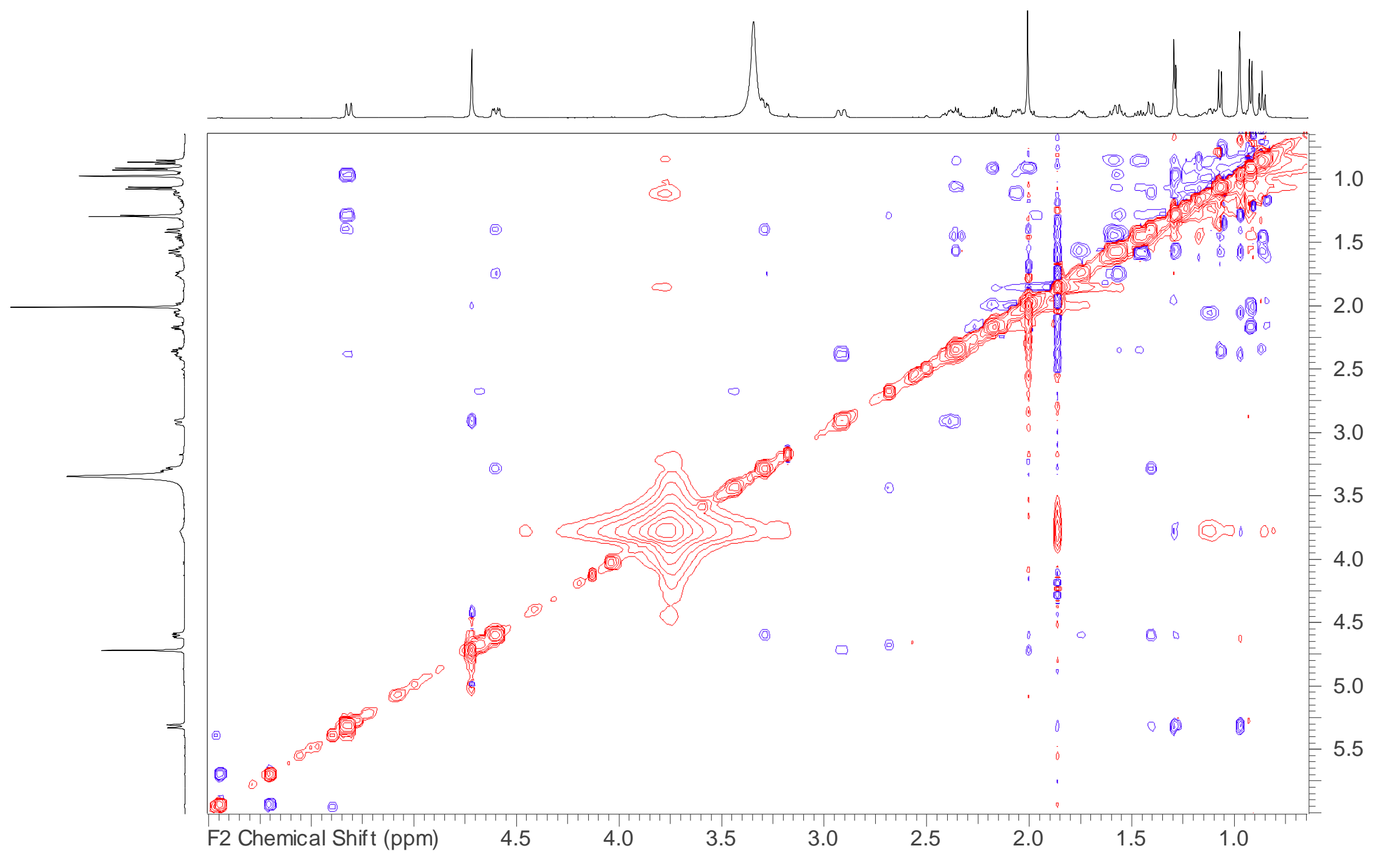

Figure S47. . ${ }^{1} \mathrm{H}-{ }^{1} \mathrm{H}$ NOESY spectrum of compounds 8 and $9\left(500 \mathrm{MHz}, \mathrm{CDCl}_{3}\right)$. 


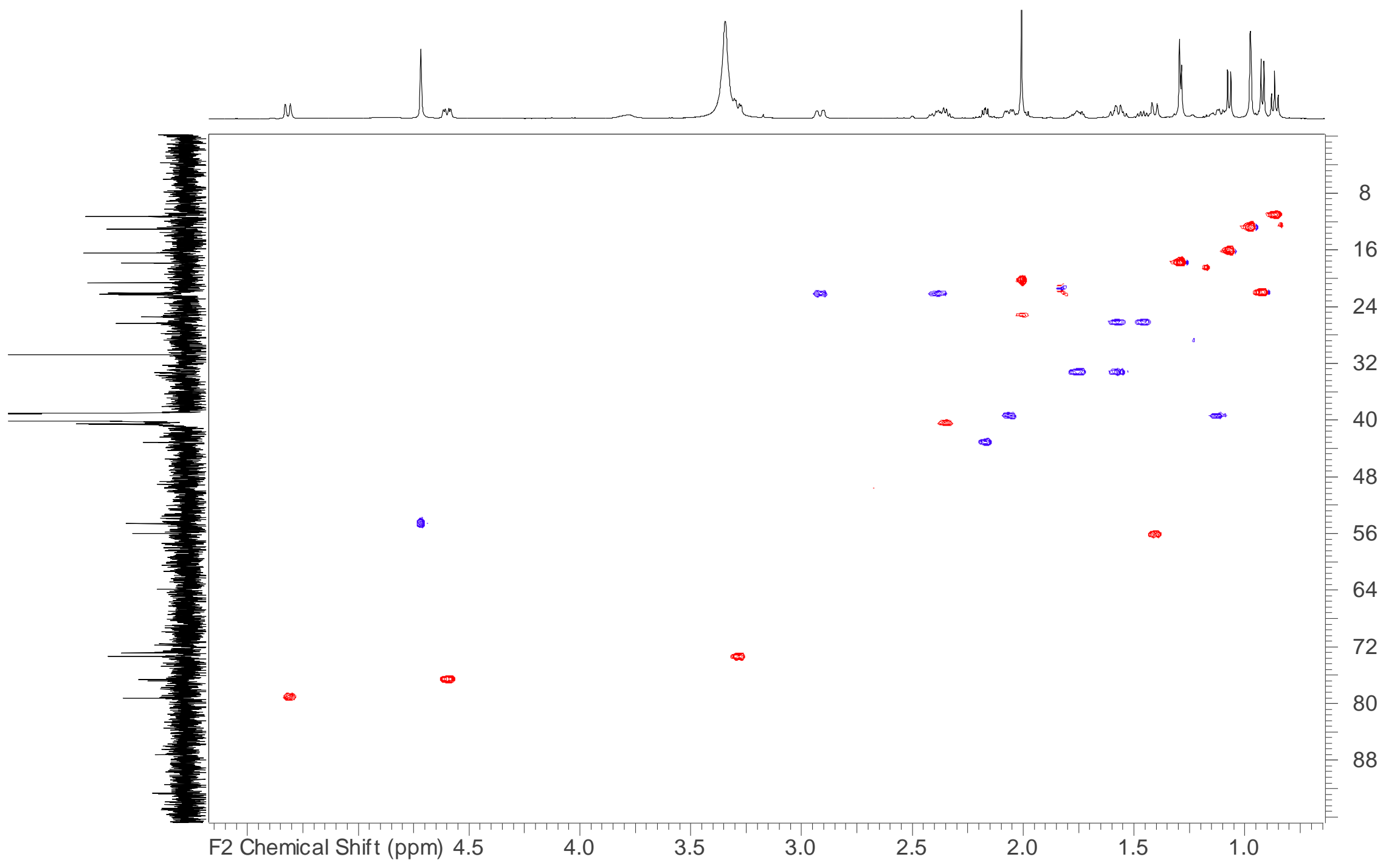

Figure S48. HSQC-DEPT spectrum of compounds 8 and $9\left(500 \mathrm{MHz}, \mathrm{CDCl}_{3}\right)$. 


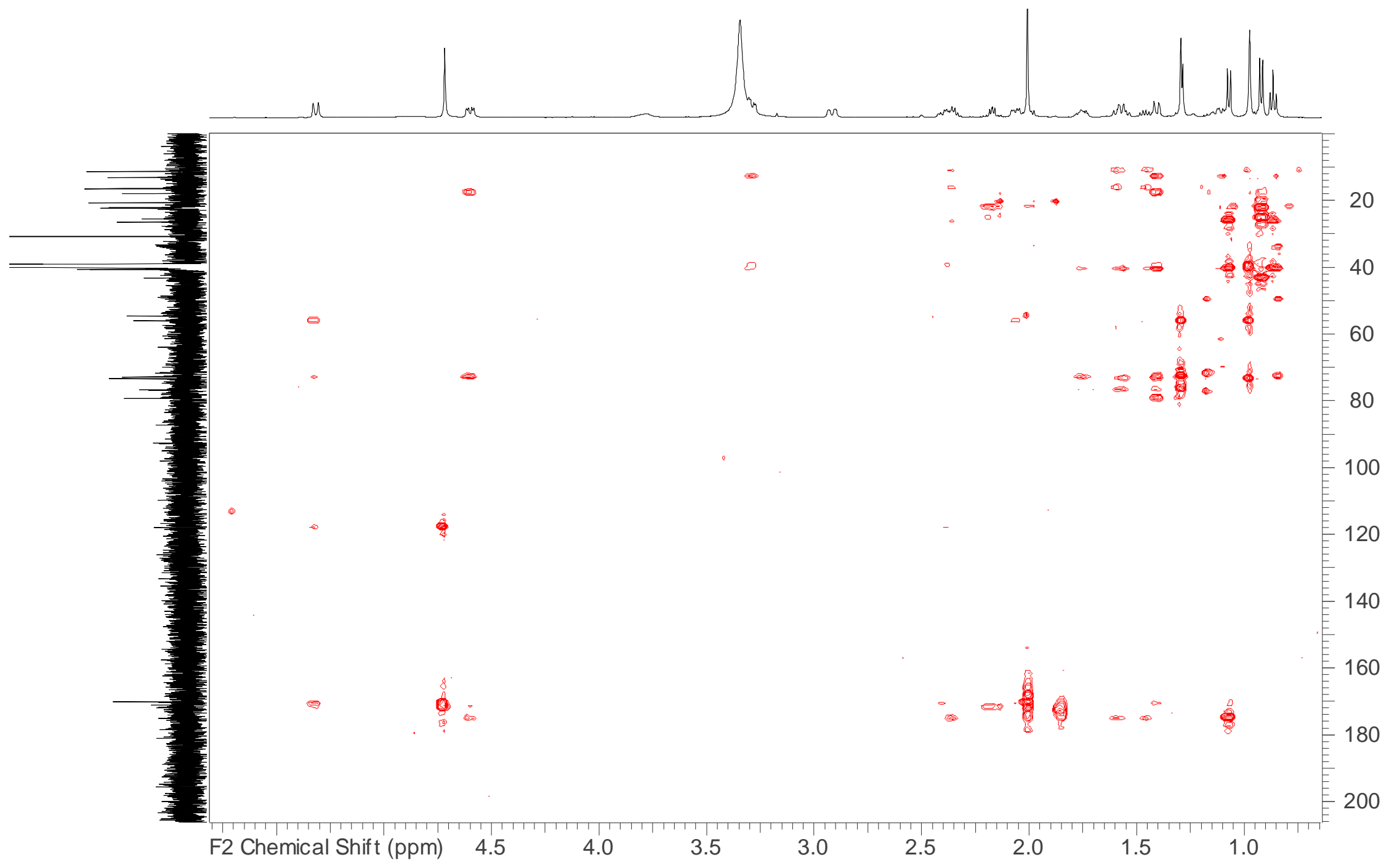

Figure S49. HMBC spectrum of compounds 8 and $9\left(500 \mathrm{MHz}, \mathrm{CDCl}_{3}\right)$. 


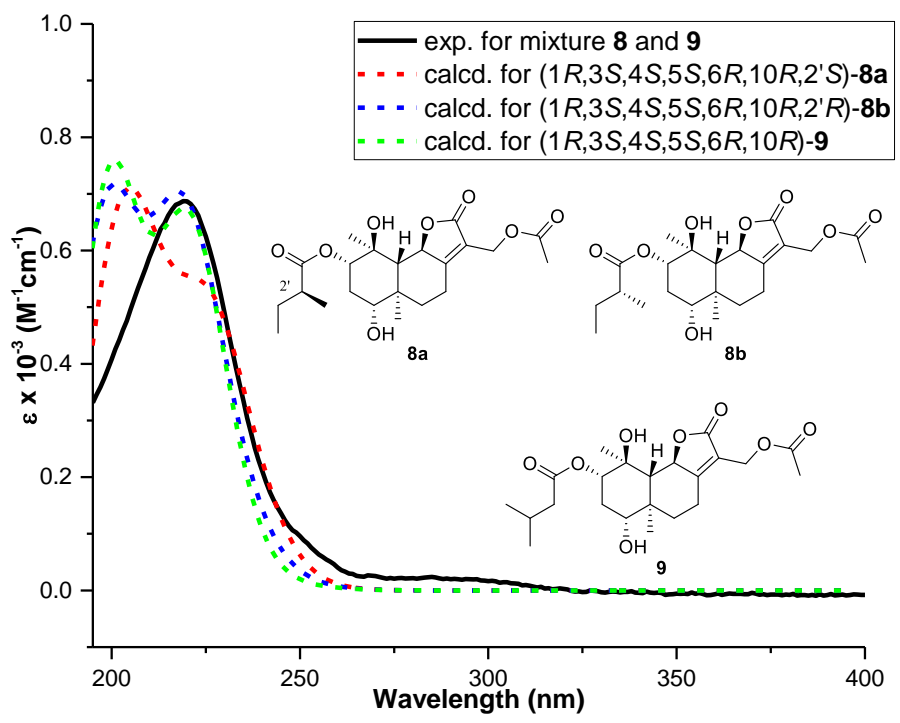

Figure S50. Comparison of experimental and computed UV spectra for compound $11 \mathrm{in} \mathrm{MeOH}$. 


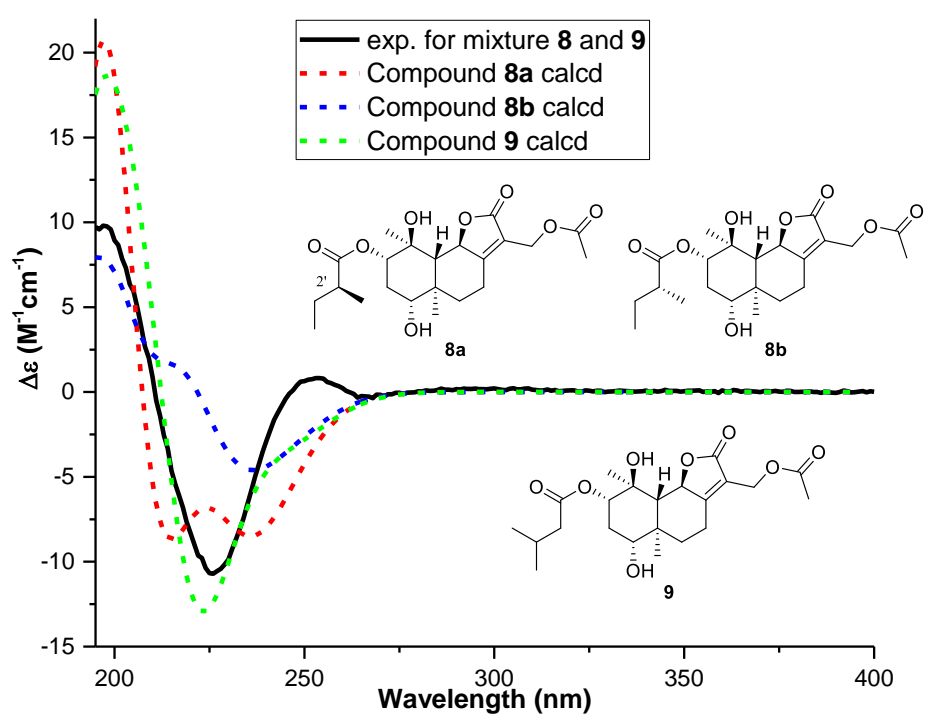

Figure S51. Comparison of the experimental ECD spectrum for the mixture of compounds $\mathbf{8}$ and 9 in $\mathrm{MeOH}$ to the computed spectra of $\mathbf{8 a}, \mathbf{8 b}$, and $\mathbf{9}$. 

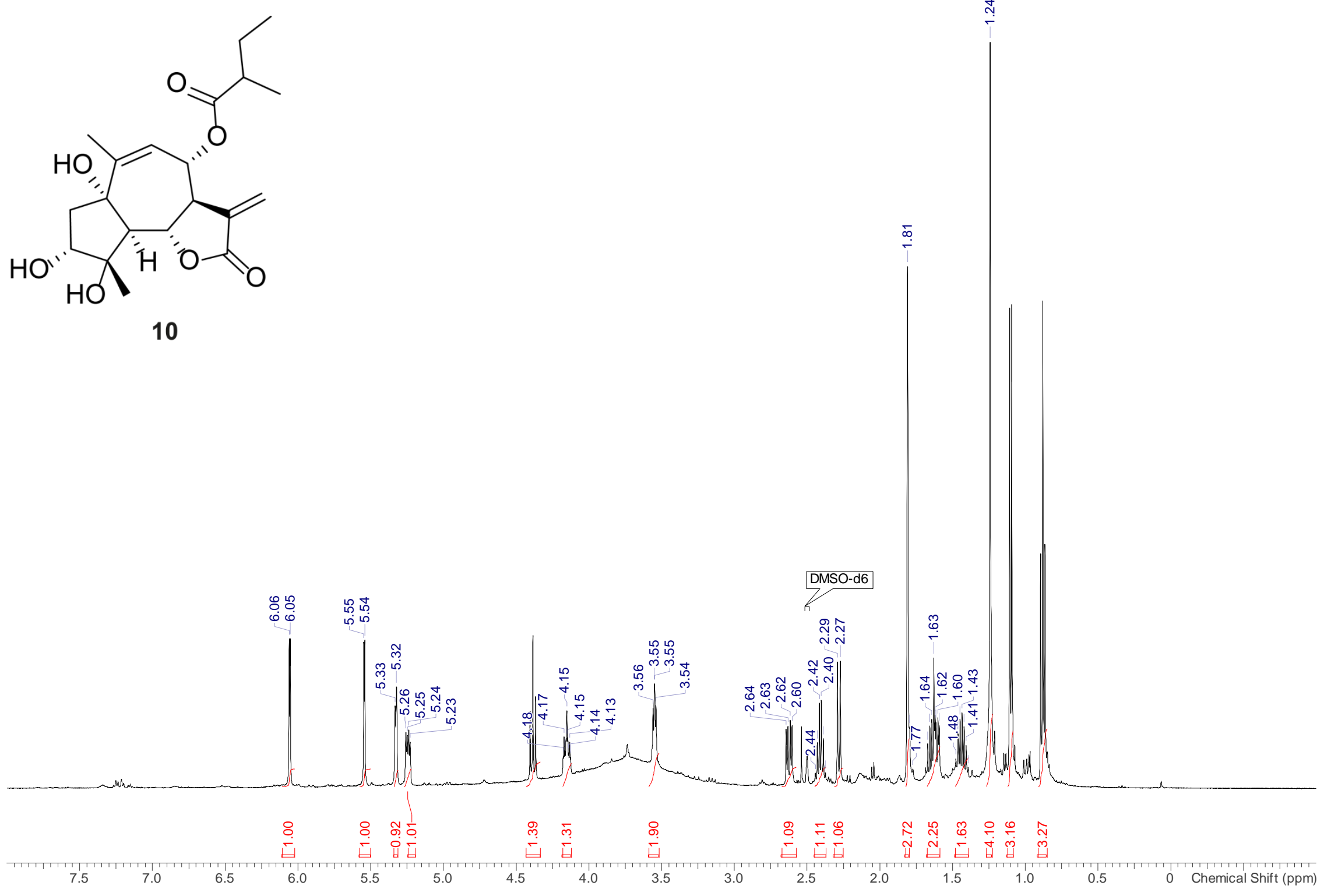

Figure S52. ${ }^{1} \mathrm{H}$ NMR spectrum of compound 10 (500 MHz, DMSO). 

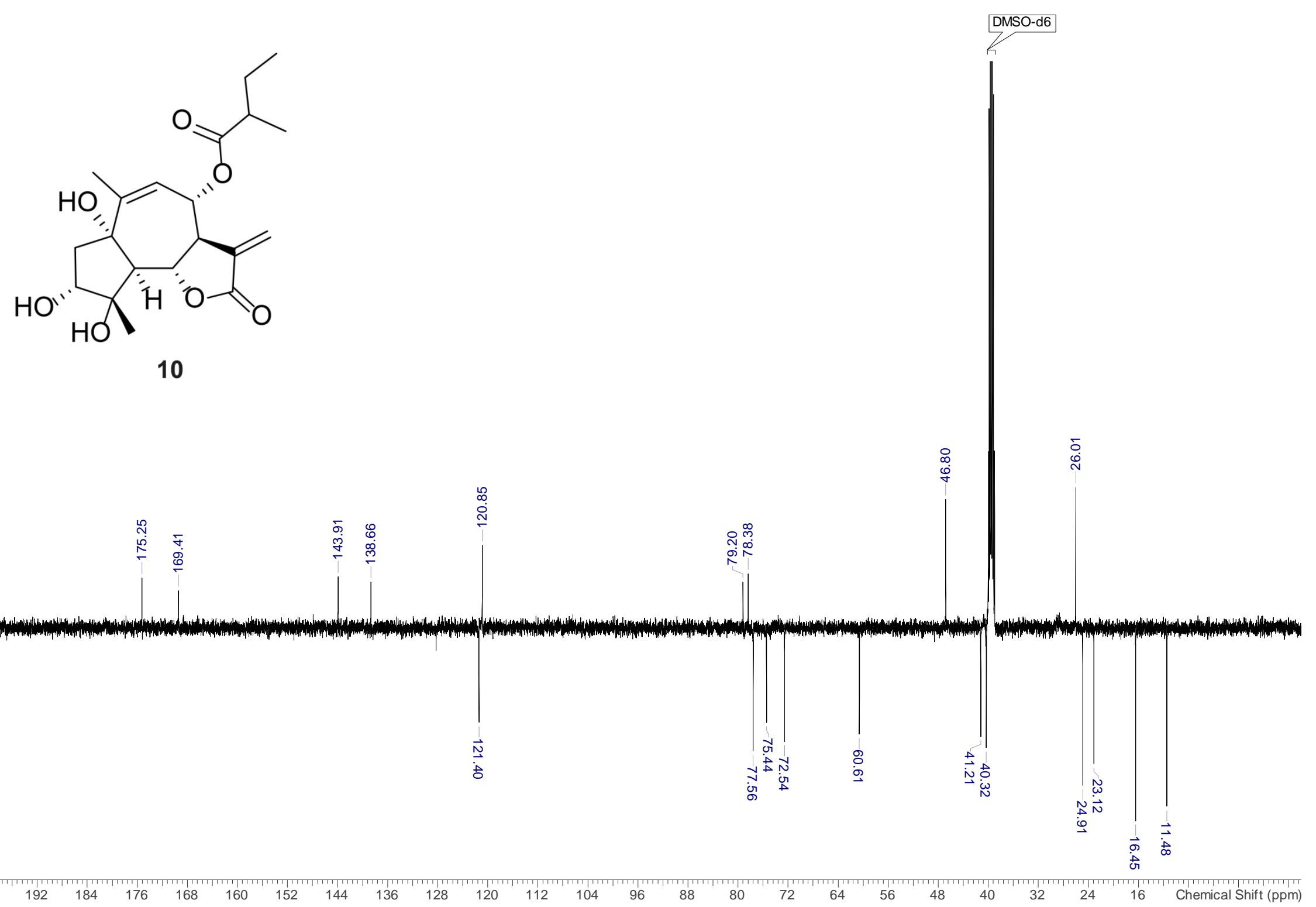

Figure S53. ${ }^{13} \mathrm{C}-\mathrm{DEPTq}$ spectrum of compound 10 (125 MHz, DMSO). 


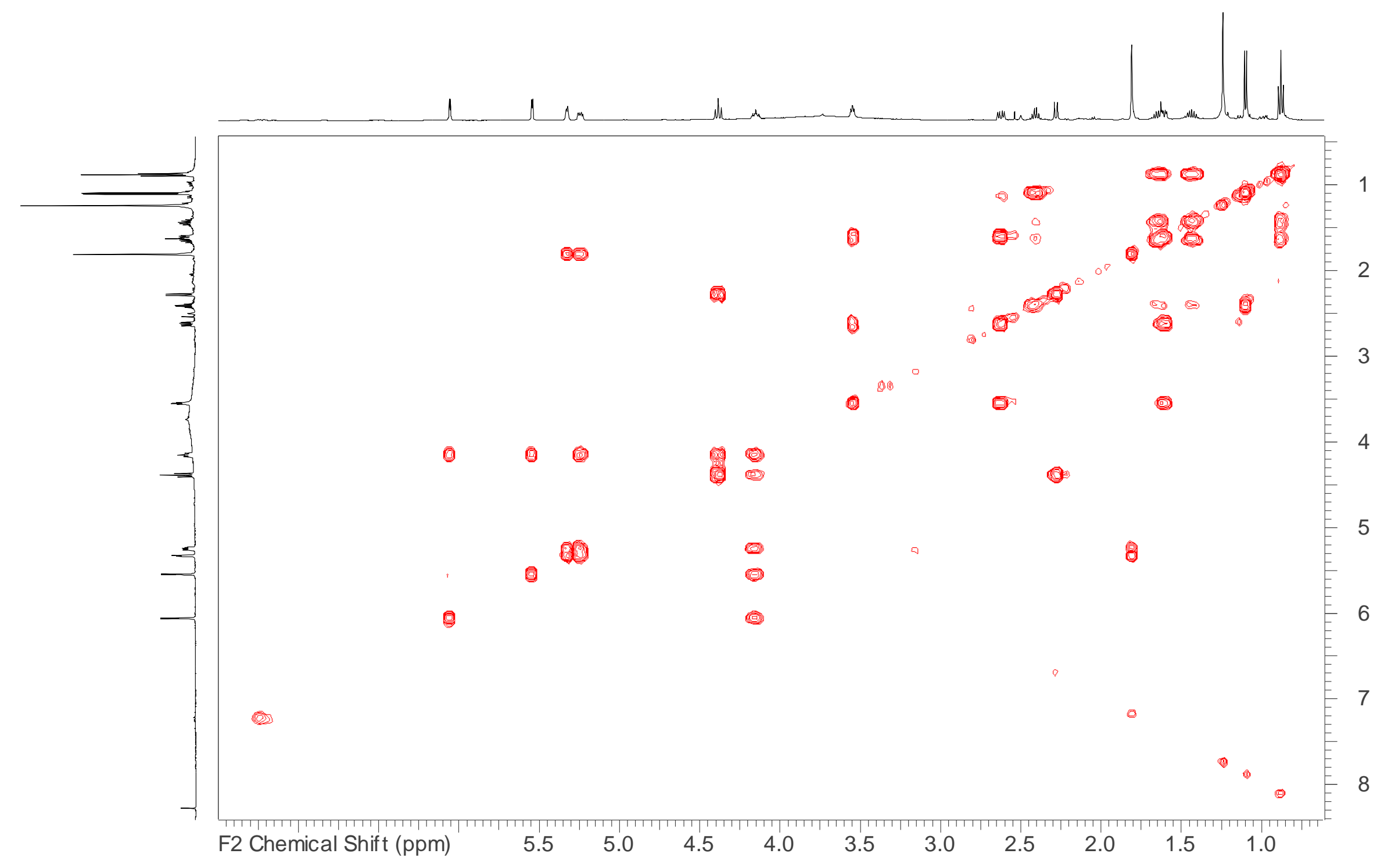

Figure S54. ${ }^{1} \mathrm{H}-{ }^{1} \mathrm{H}$ COSY spectrum of compound 10 (500 MHz, DMSO). 


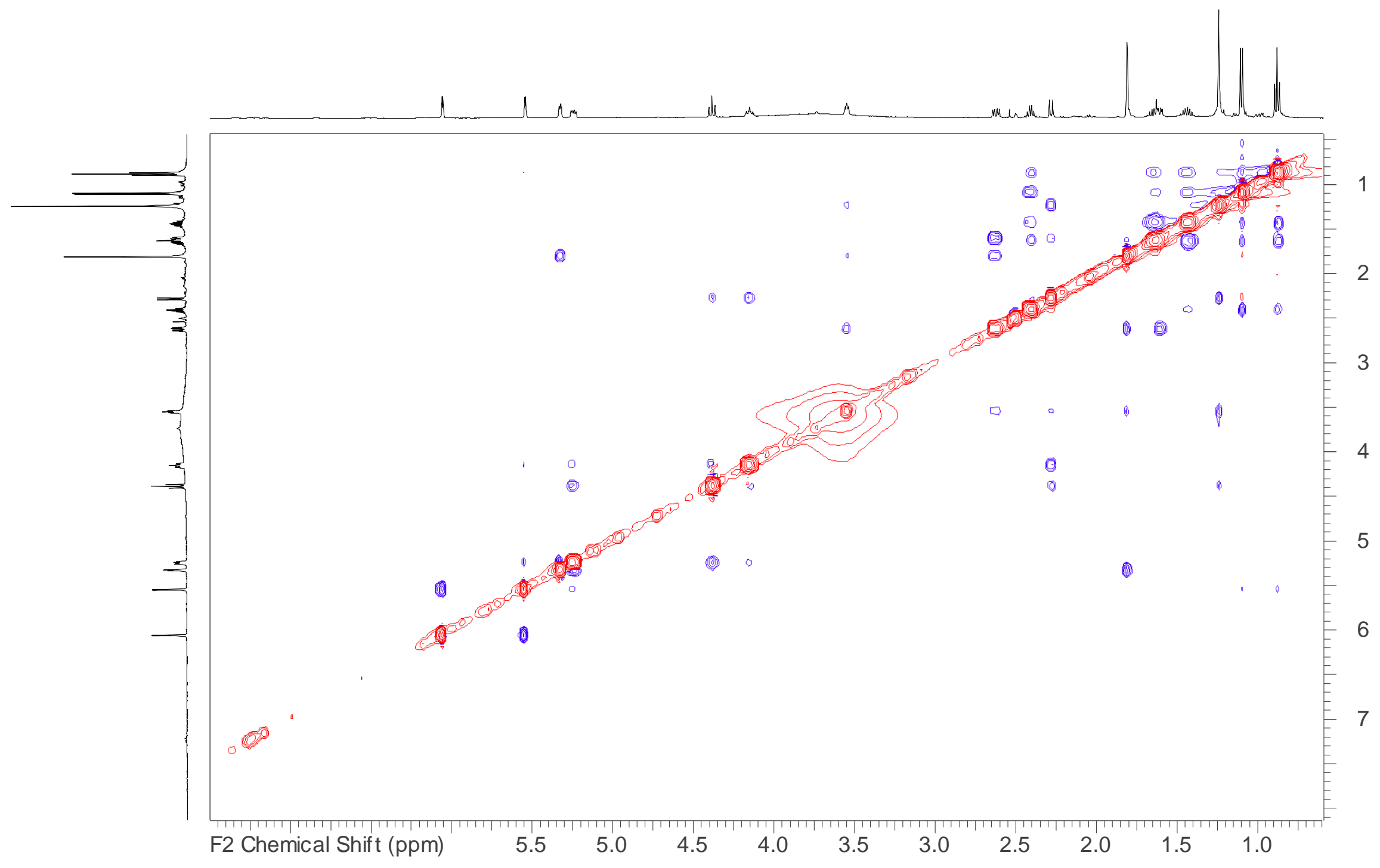

Figure S55. ${ }^{1} \mathrm{H}-{ }^{1} \mathrm{H}$ NOESY spectrum of compound 10 (500 MHz, DMSO). 


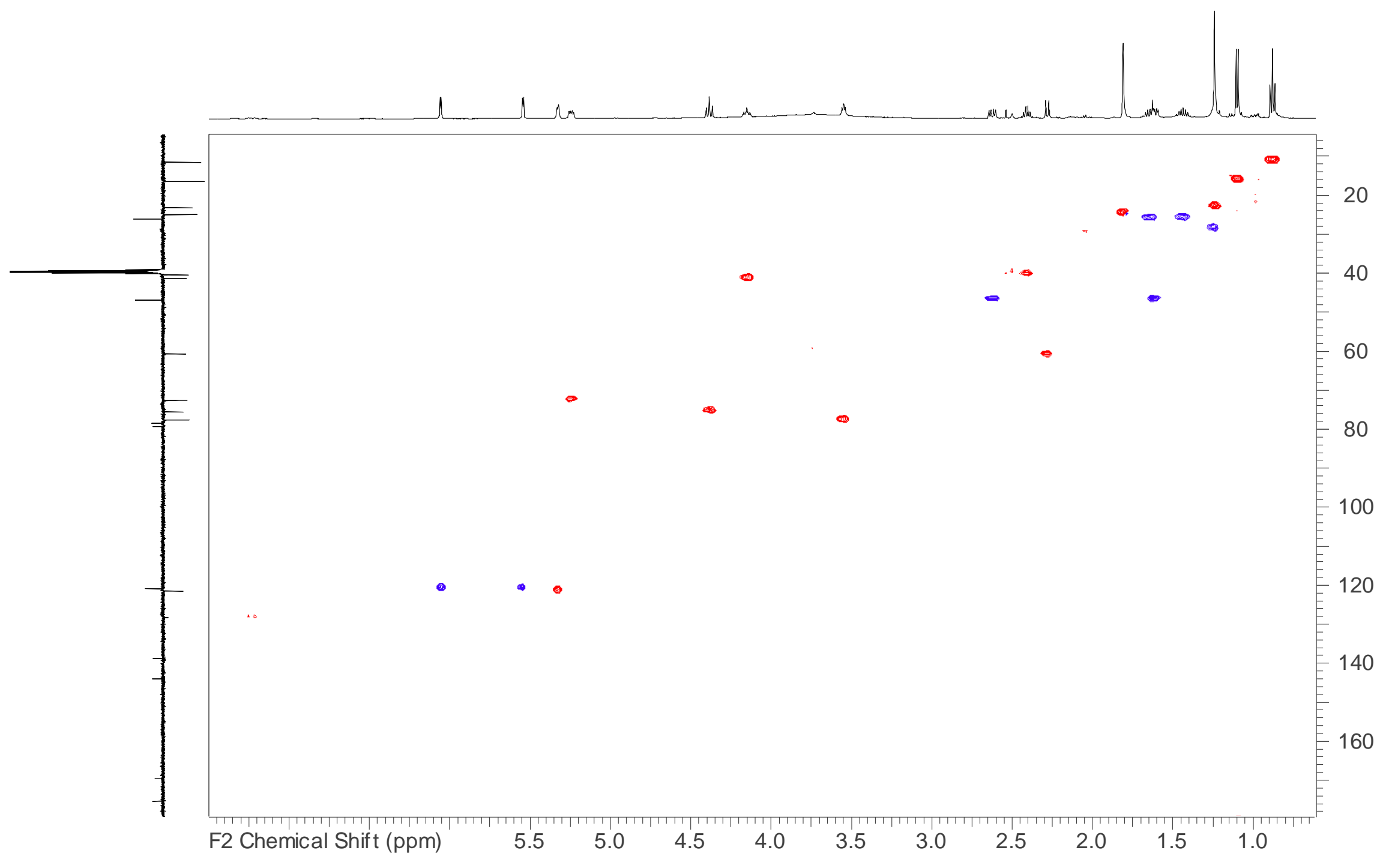

Figure S56 HSQC-DEPT spectrum of compound 10 (500 MHz, DMSO). 


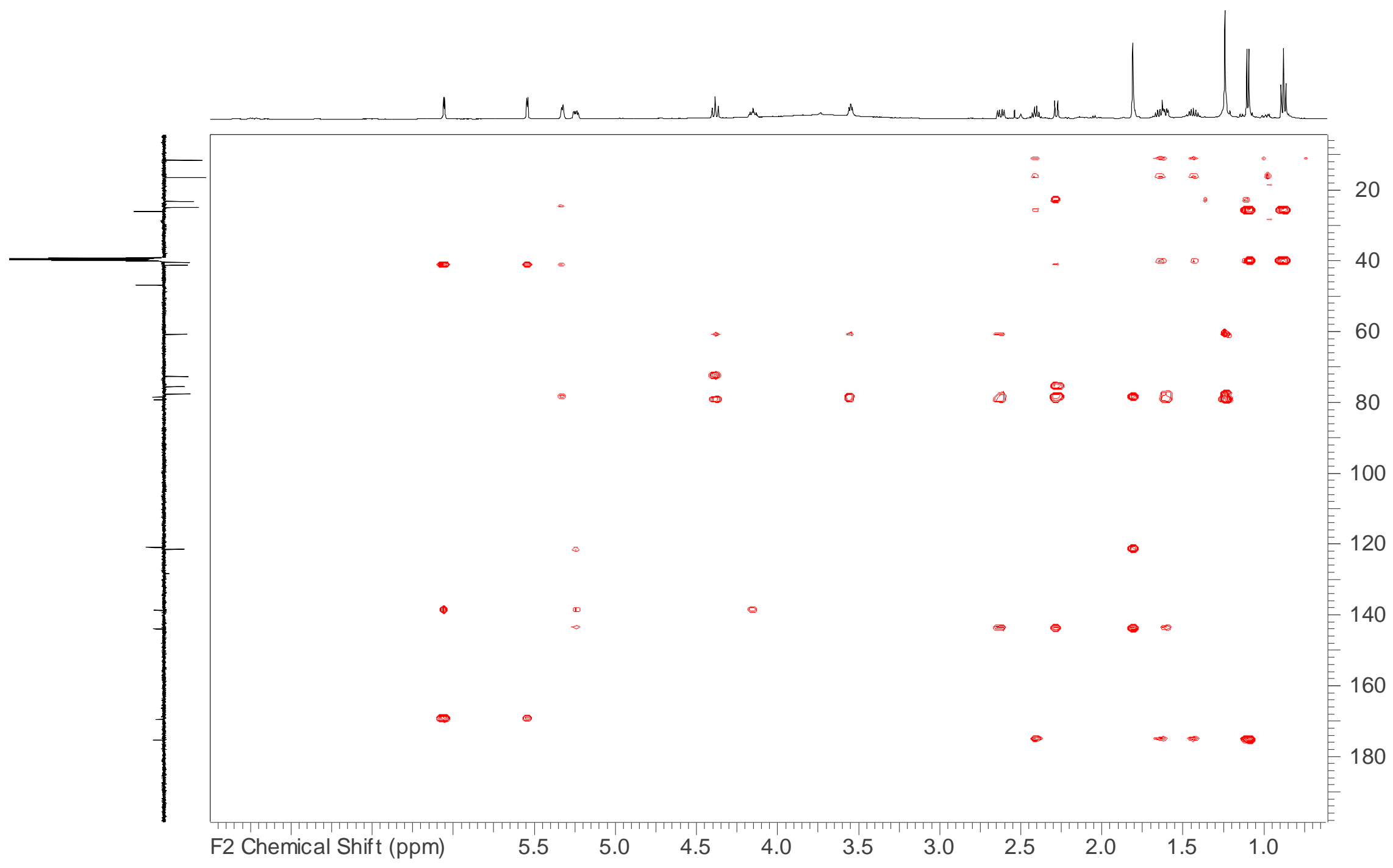

Figure S57. HMBC spectrum of compound 10 (500 MHz, DMSO). 


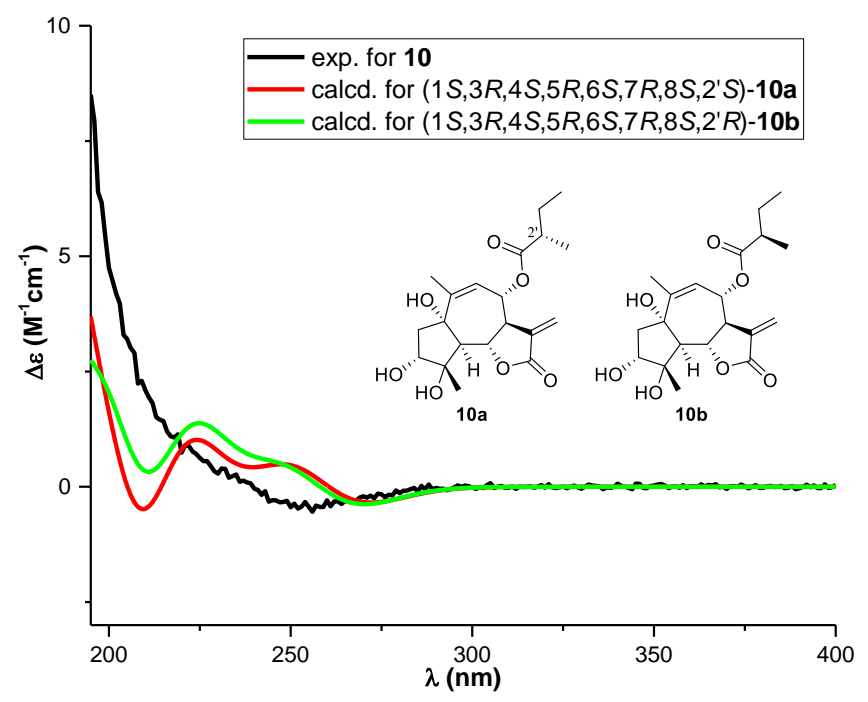

Figure S58. Comparison of the experimental ECD spectrum for compound $\mathbf{1 0}$ in $\mathrm{MeOH}$ to the computed spectra of $\mathbf{1 0 a}$ and $\mathbf{1 0 b}$. 


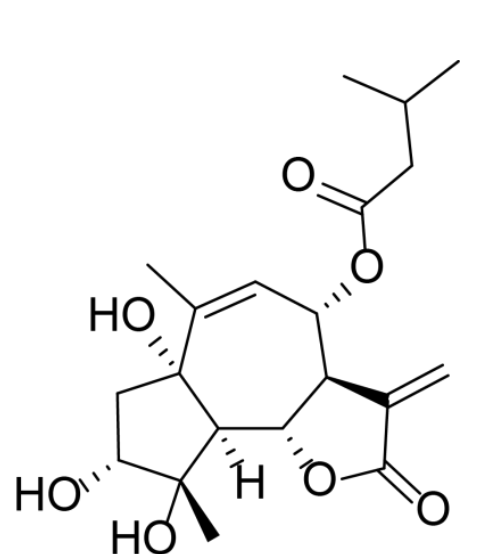

11

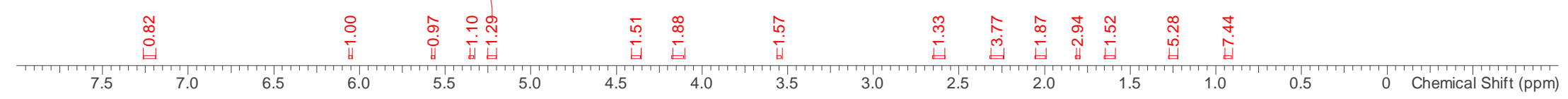

Figure S59. ${ }^{1} \mathrm{H}$ NMR spectrum of compound 11 (500 MHz, DMSO). 


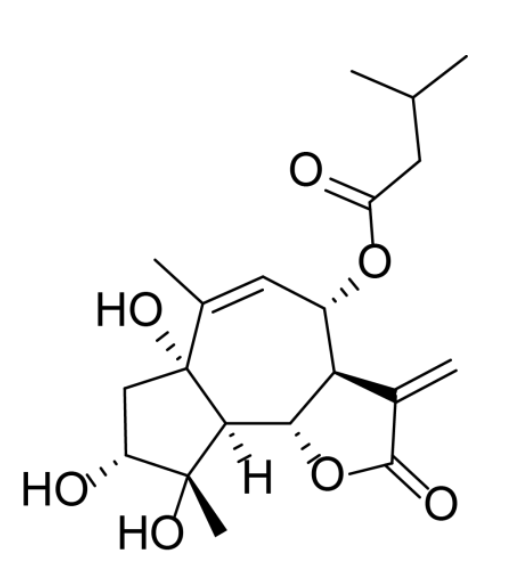

11
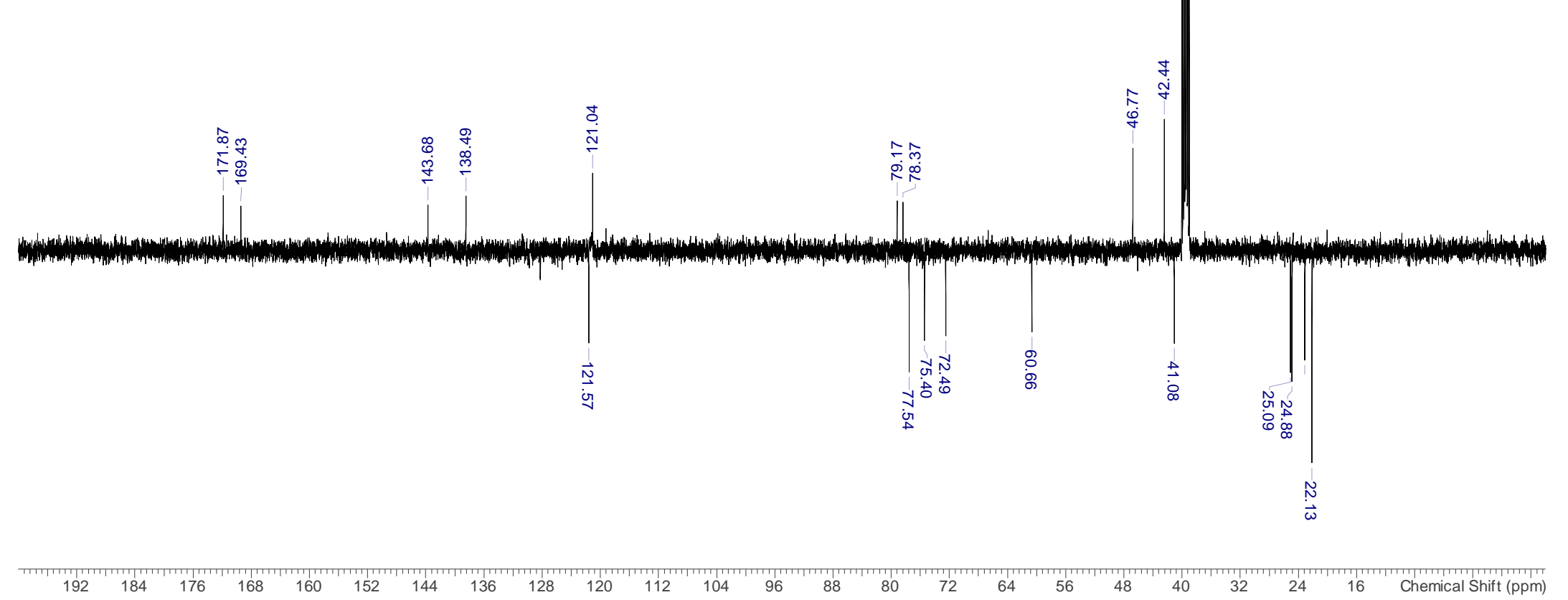

Figure S60. ${ }^{13} \mathrm{C}$-DEPTq spectrum of compound 11 (125 MHz, DMSO). 


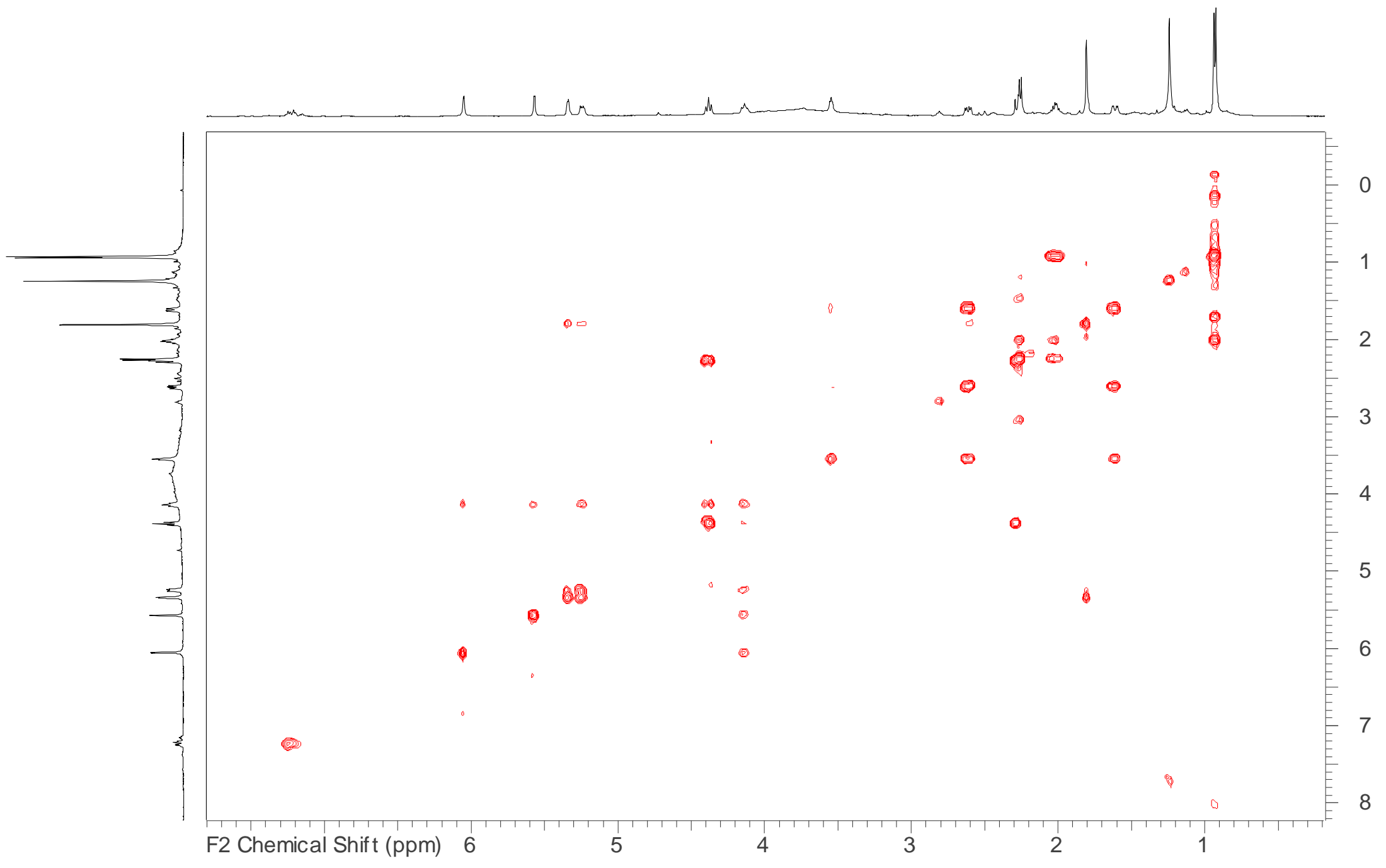

Figure S61. ${ }^{1} \mathrm{H}-{ }^{1} \mathrm{H}$ COSY spectrum of compound 11 (500 MHz, DMSO). 


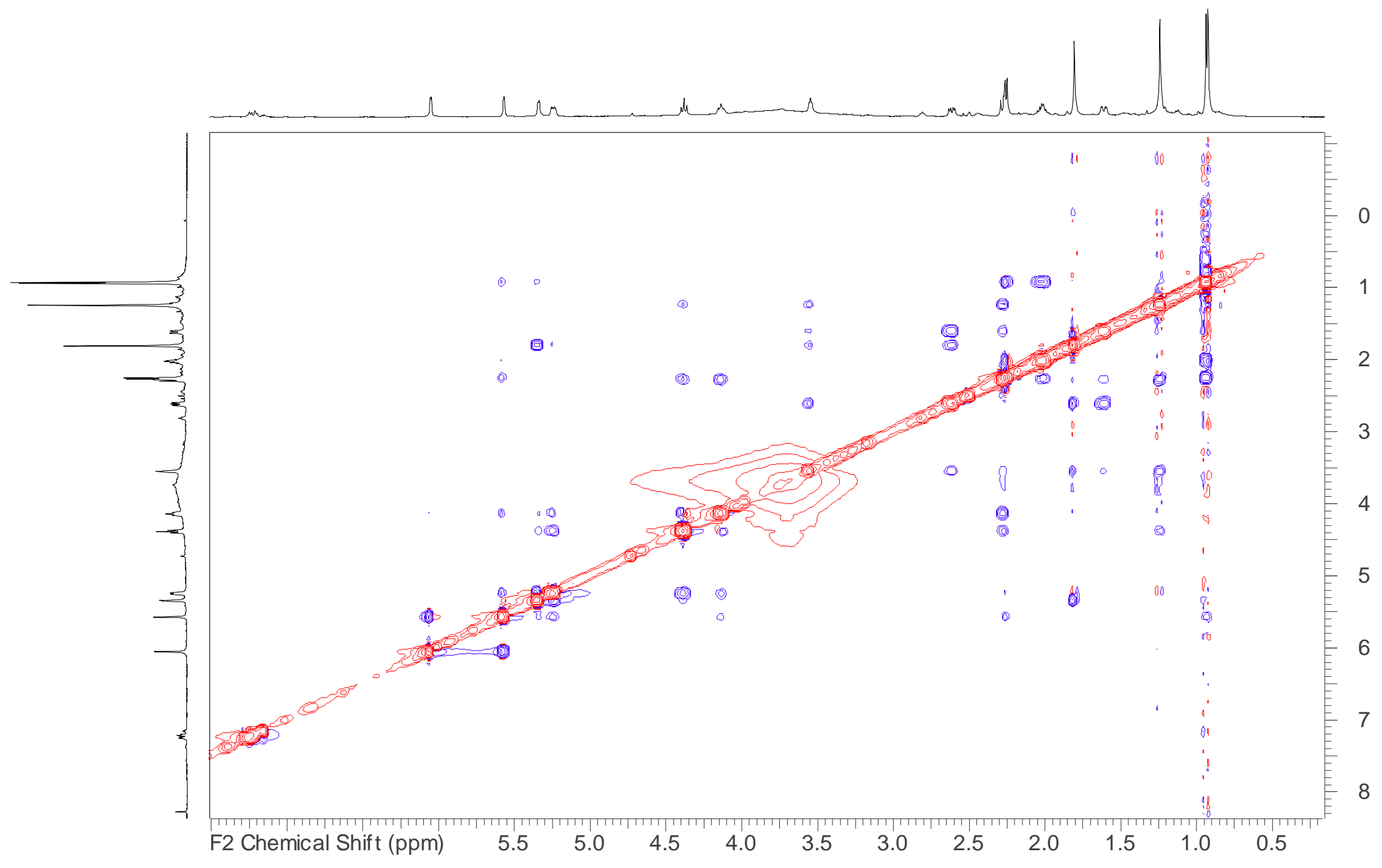

Figure S62. ${ }^{1} \mathrm{H}-{ }^{1} \mathrm{H}$ NOESY spectrum of compound 11 (500 MHz, DMSO). 


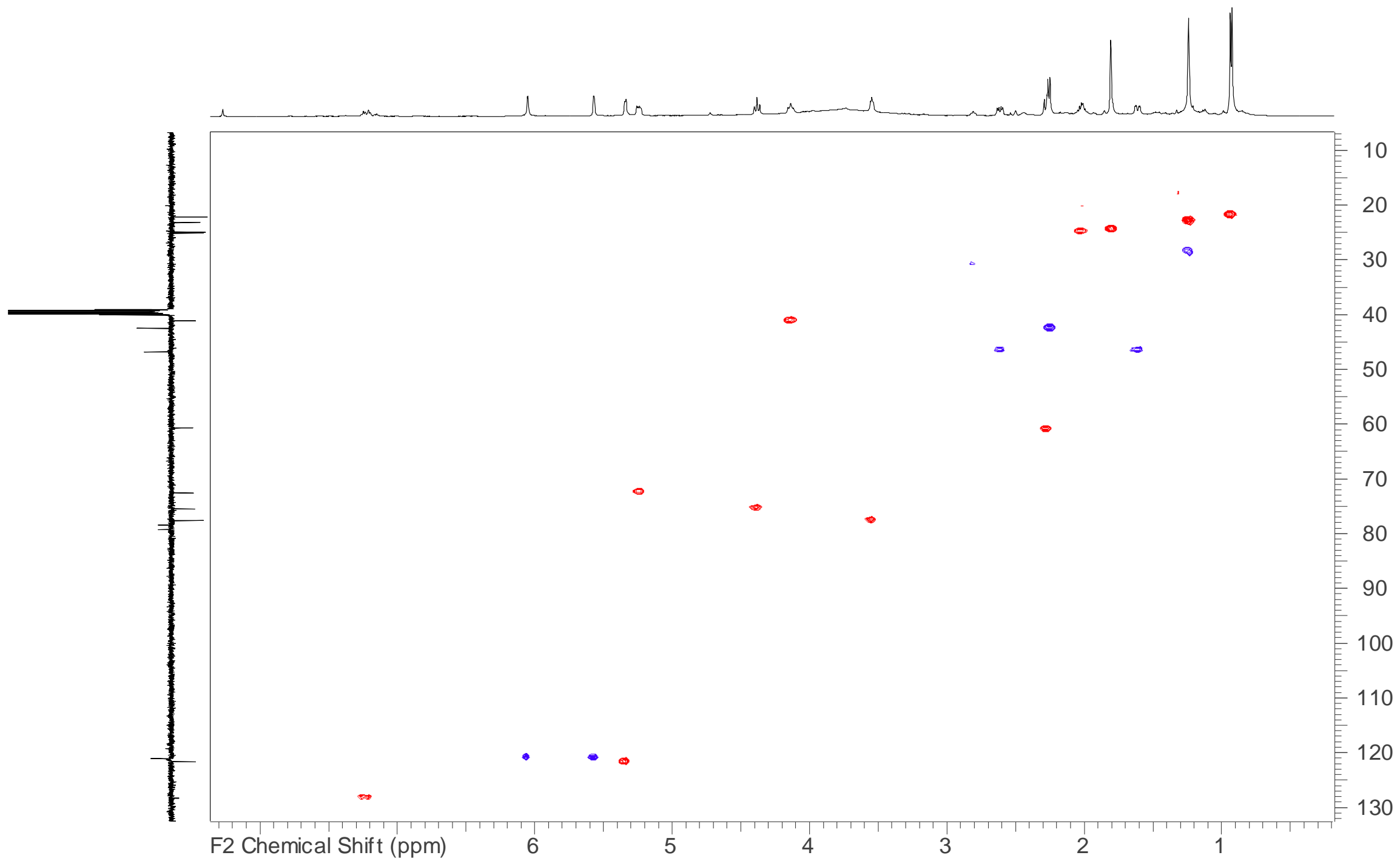

Figure S63. HSQC-DEPT spectrum of compound 11 (500 MHz, DMSO). 


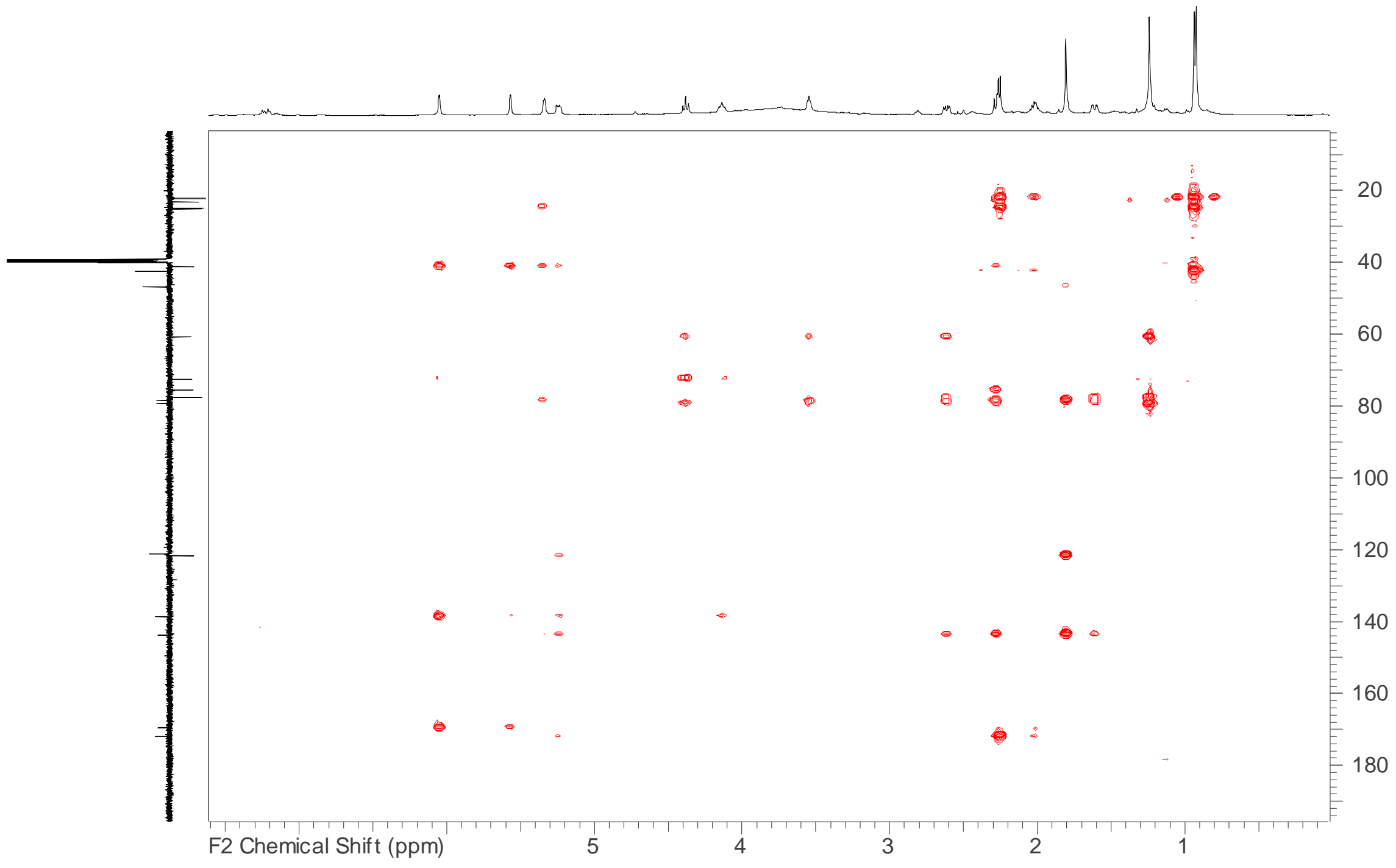

Figure S64. HMBC spectrum of compound 11 (500 MHz, DMSO). 


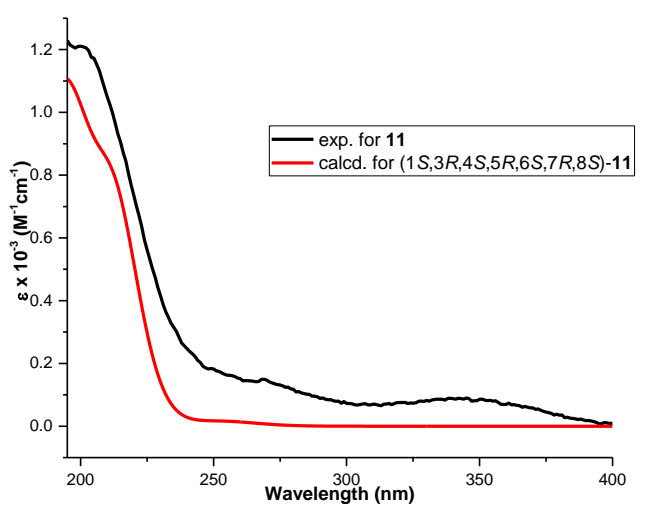

Figure S65. Comparison of experimental and computed UV spectra for compound 11 in $\mathrm{MeOH}$. 


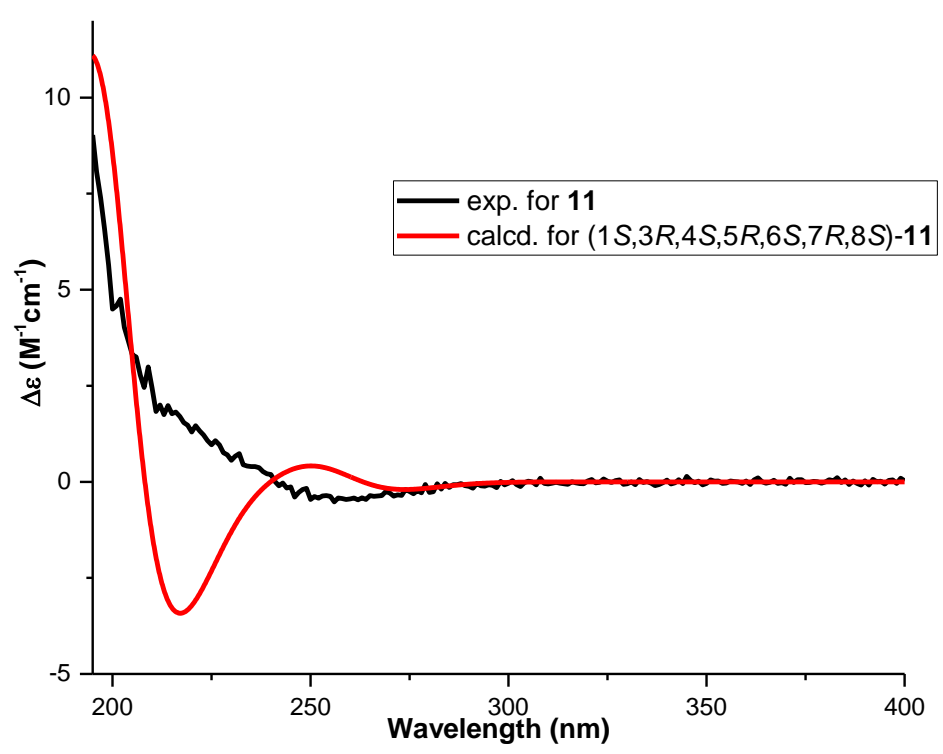

Figure S66. Comparison of experimental and computed ECD spectra for compound 11 in $\mathrm{MeOH}$. 


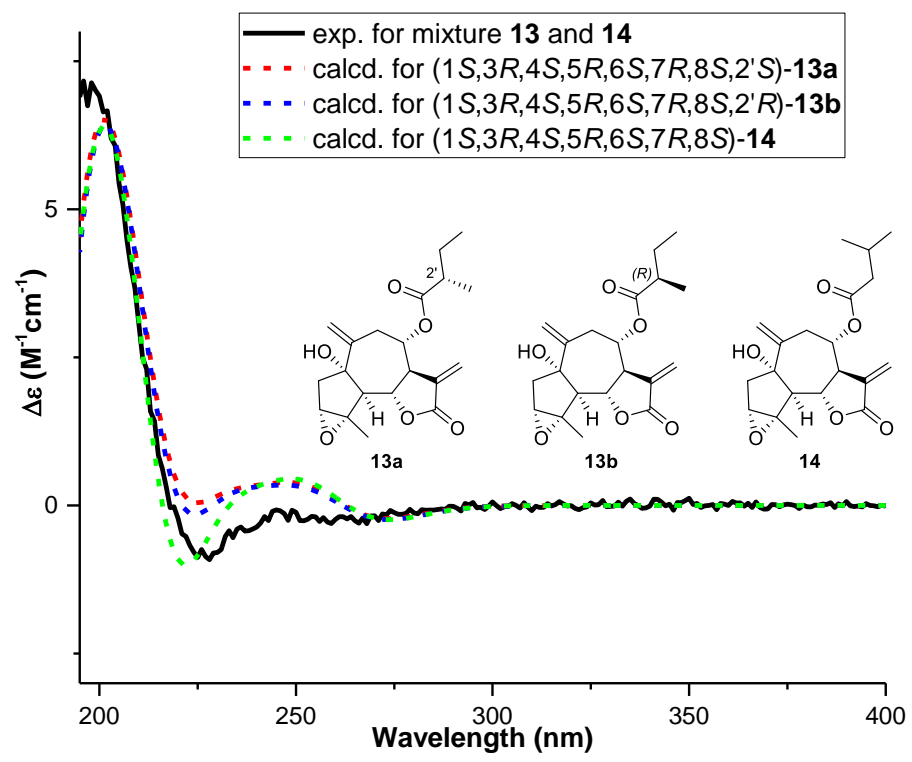

Figure S67. Comparison of experimental ECD spectrum of the mixture of compounds $\mathbf{1 3}$ and $\mathbf{1 4} \mathrm{in} \mathrm{MeOH}(270 \mu \mathrm{g} / \mathrm{mL})$ to the computed spectra of 13a, 13b, and 14. Combined curves were mixed in the ratio of 1:1 as determined between compounds $\mathbf{1 3}$ and $\mathbf{1 4}$ via ${ }^{1} \mathrm{H}$ NMR. 


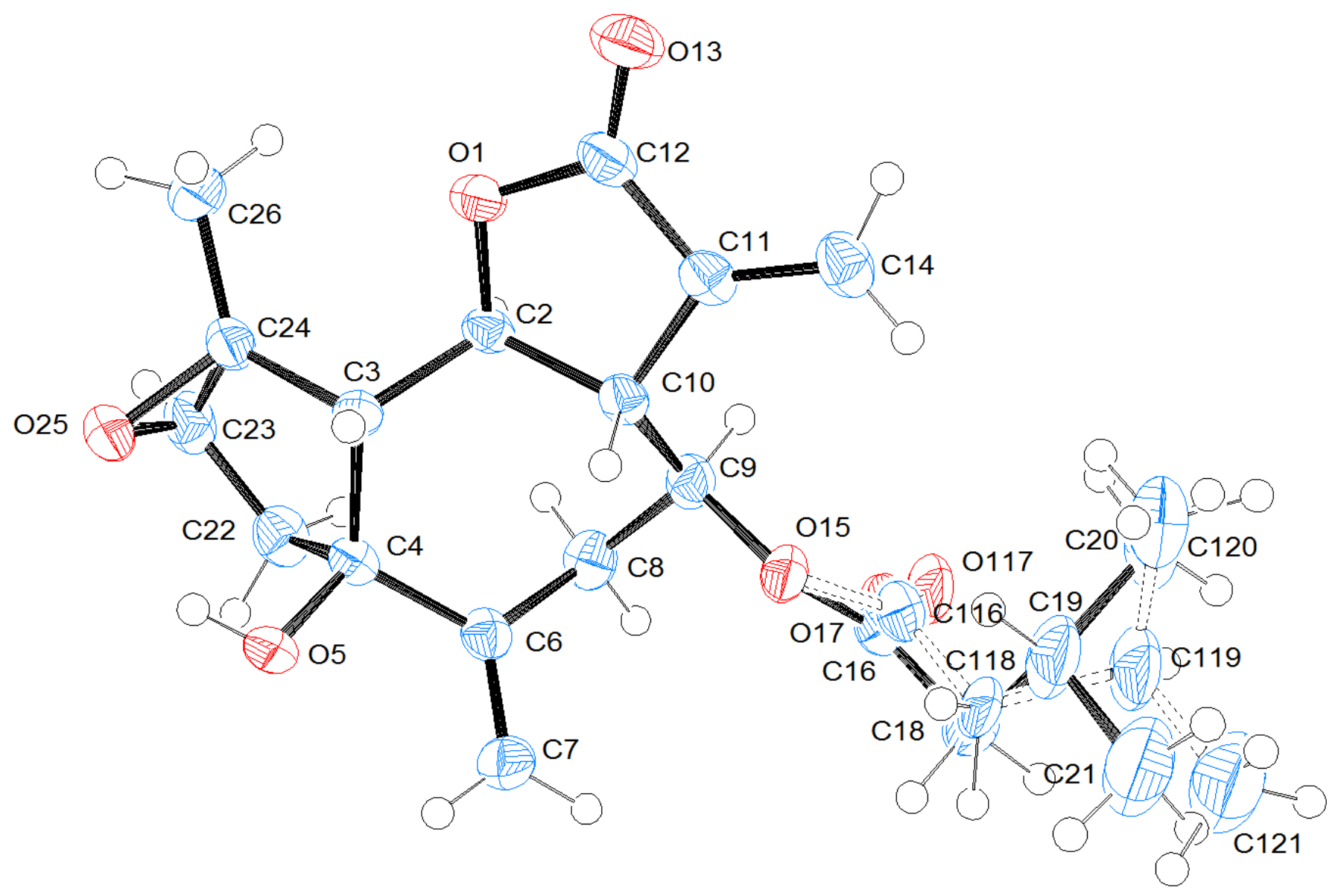

Figure S68. ORTEP diagram of compound 14 from the X-ray diffraction experiment. 
Table S7. Crystal data for 14 and $\mathbf{1 5 .}$

\begin{tabular}{lll} 
formula & $\mathbf{1 4}$ & $\mathbf{1 5}$ \\
formula weight & $\mathrm{C}_{20} \mathrm{H}_{26} \mathrm{O}_{6}$ & $\mathrm{C}_{17} \mathrm{H}_{20} \mathrm{O}_{5}$ \\
$Z$, calculated density & 362.42 & 304.34 \\
$\mathrm{~F}(000)$ & $4,1.272 \mathrm{Mg} * \mathrm{~m}^{-3}$ & $4,1.263 \mathrm{Mg}^{*} \mathrm{~m}^{-3}$ \\
description and size of crystal & 775.997 & 647.996 \\
absorption coefficient & colorless block, $0.11 * 0.13 * 0.21 \mathrm{~mm}^{3}$ & colorless block, $0.05 * 0.09 * 0.14 \mathrm{~mm}^{3}$ \\
min/max transmission & 0.769 & 0.487 \\
temperature & $0.90 / 0.92$ & $0.98 / 0.98$ \\
radiation (wavelength) & $123 \mathrm{~K}$ & $123 \mathrm{~K}$ \\
Crystal system, space group & $\mathrm{Cu} K_{\alpha}(\hat{\mathrm{I}}=1.54178 \AA)$ & $\mathrm{Ga} K_{\alpha}(\mathrm{I}=1.34143 \AA)$ \\
a & orthorhombic, $\mathrm{P} 2{ }_{1} 2_{1} 2_{1}$ & orthorhombic, $\mathrm{P} 22_{1} 2{ }_{1} 2$ \\
$\mathrm{~b}$ & $9.1487(7) \AA$ & $19.8206(4) \AA$ \\
$\mathrm{c}$ & $10.3308(7) \AA$ & $9.5646(2) \AA$ \\
$\hat{\mathrm{I}}^{ \pm}$ & $20.0261(14) \AA$ & $8.4390(2) \AA$ \\
$\hat{\mathrm{I}}^{2}$ & $90^{\circ}$ & $90^{\circ}$ \\
$\hat{\mathrm{I}}^{3}$ & $90^{\circ}$ & $90^{\circ}$ \\
$\mathrm{V}$ & $90^{\circ}$ & $90^{\circ}$ \\
min/max $\hat{\mathrm{I}}^{\sim}$ & $1892.7(2) \AA^{3}$ & $1599.83(6) \AA^{3}$ \\
number of collected reflections & $4.816 \AA / 68.964 \AA$ & $3.881 \AA / 57.257 \AA$ \\
number of independent reflections & 25073 & 33193 \\
number of observed reflections & $3419(\mathrm{merging} \mathrm{r}=0.032)$ & $3101(\mathrm{merging} \mathrm{r}=0.024)$ \\
number of refined parameters & $3485 \mathrm{I}>2.0 \backslash \sigma(\mathrm{I})$ & $3274 \mathrm{I}>2.0 \backslash \sigma(\mathrm{I})$ \\
$\mathrm{R}$ & 295 & 200 \\
$\mathrm{R}_{\mathrm{w}}$ & 0.0473 & 0.0265 \\
goodness of fit & 0.0496 & 0.0294 \\
Flack parameter & 1.0832 & 1.1151 \\
\hline & $0.10(19)$ & $0.06(15)$ \\
\hline
\end{tabular}




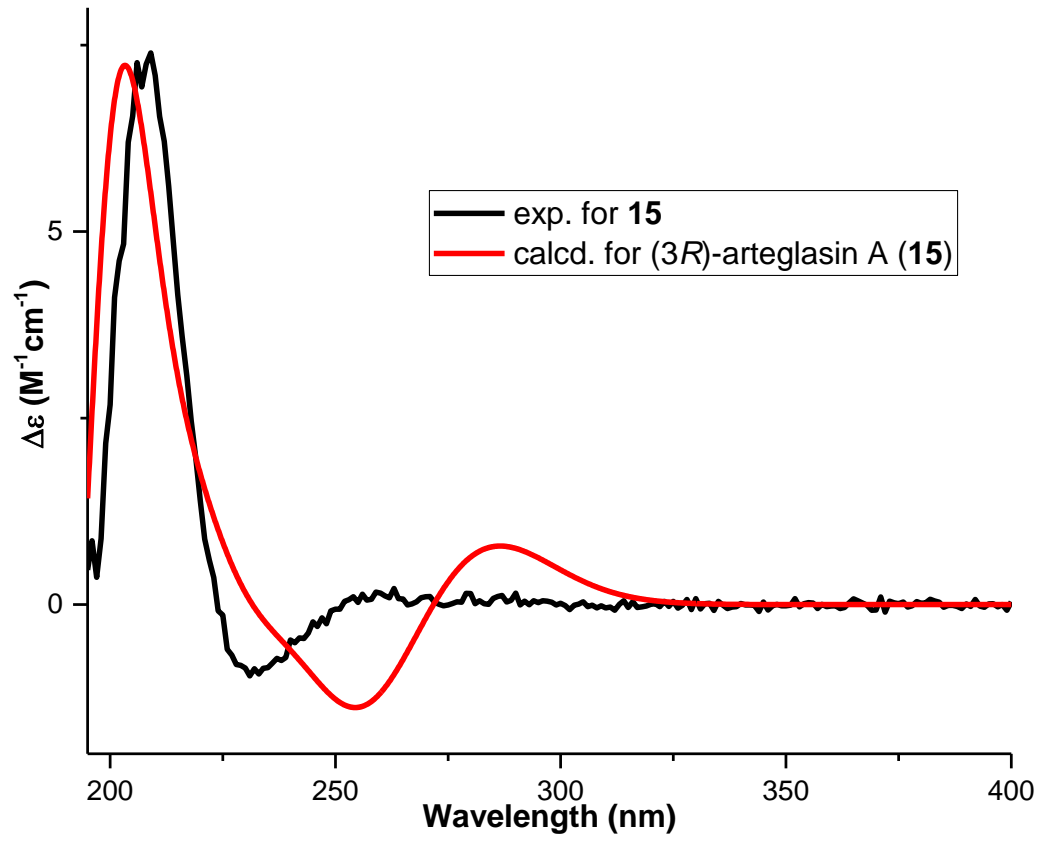

Figure S69. Comparison of experimental and computed ECD spectra for compound 15 in $\mathrm{MeOH}$. 


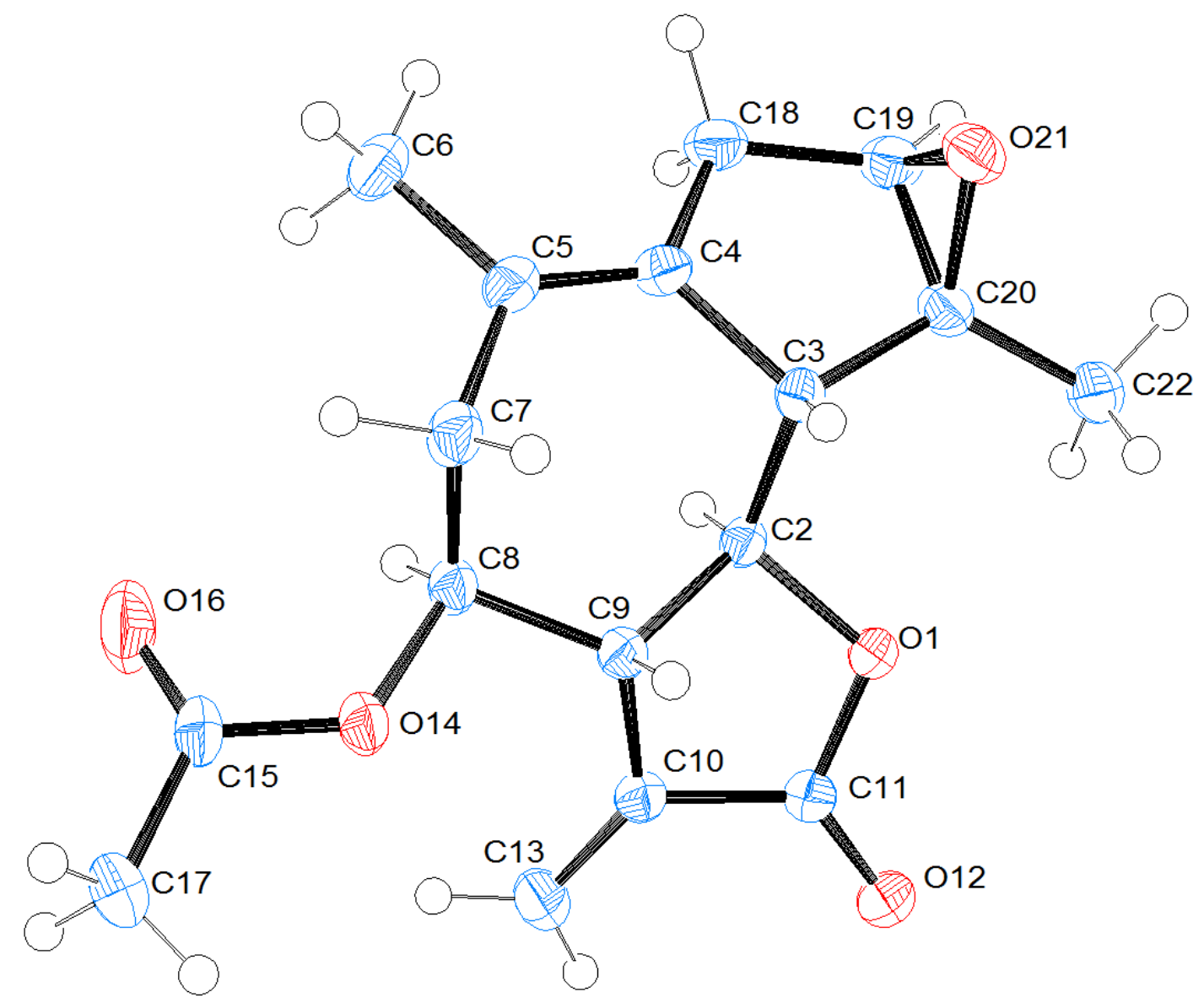

Figure S70. ORTEP diagram of compound 15 from the X-ray diffraction experiment. 


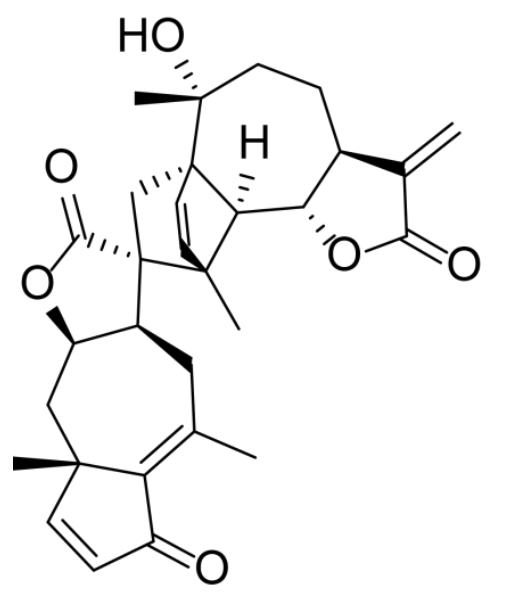

17

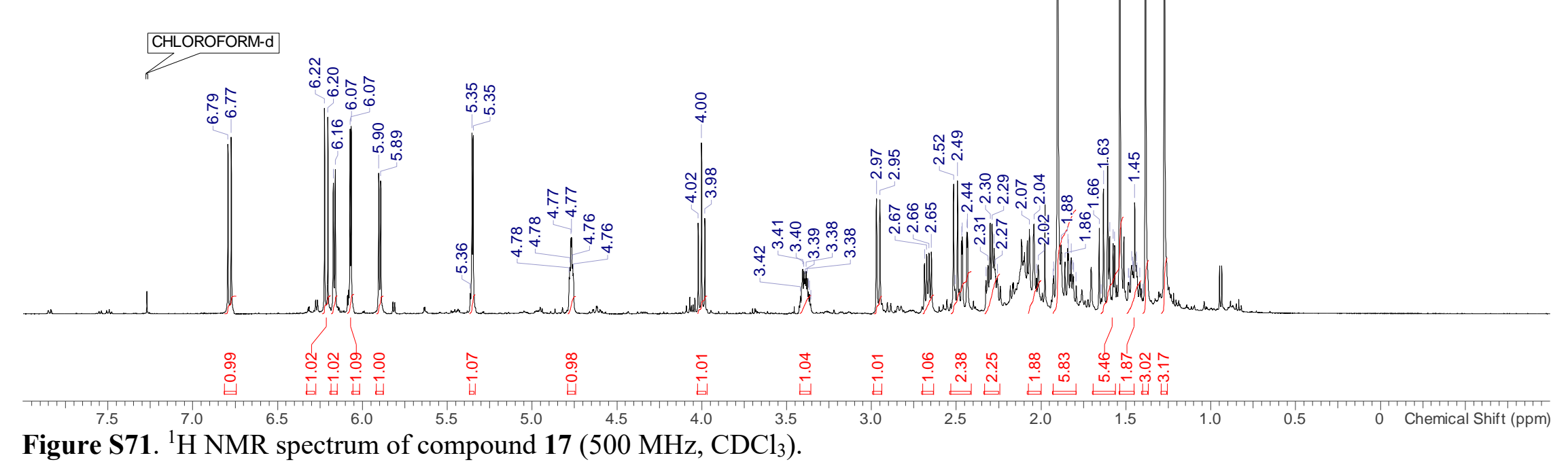

Figure S71. ${ }^{1} \mathrm{H}$ NMR spectrum of compound $17\left(500 \mathrm{MHz}, \mathrm{CDCl}_{3}\right)$. 


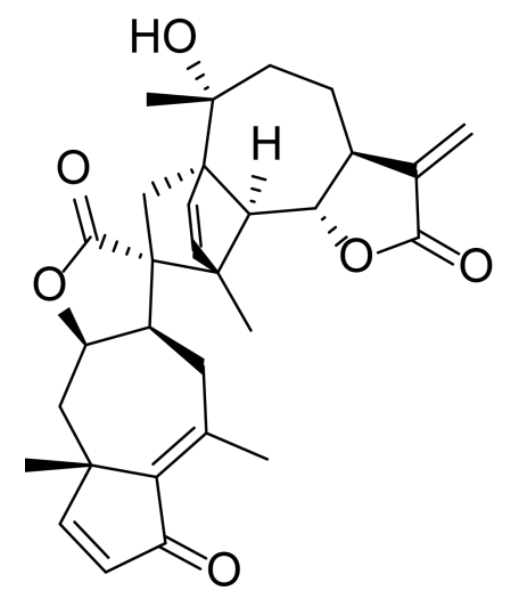

17

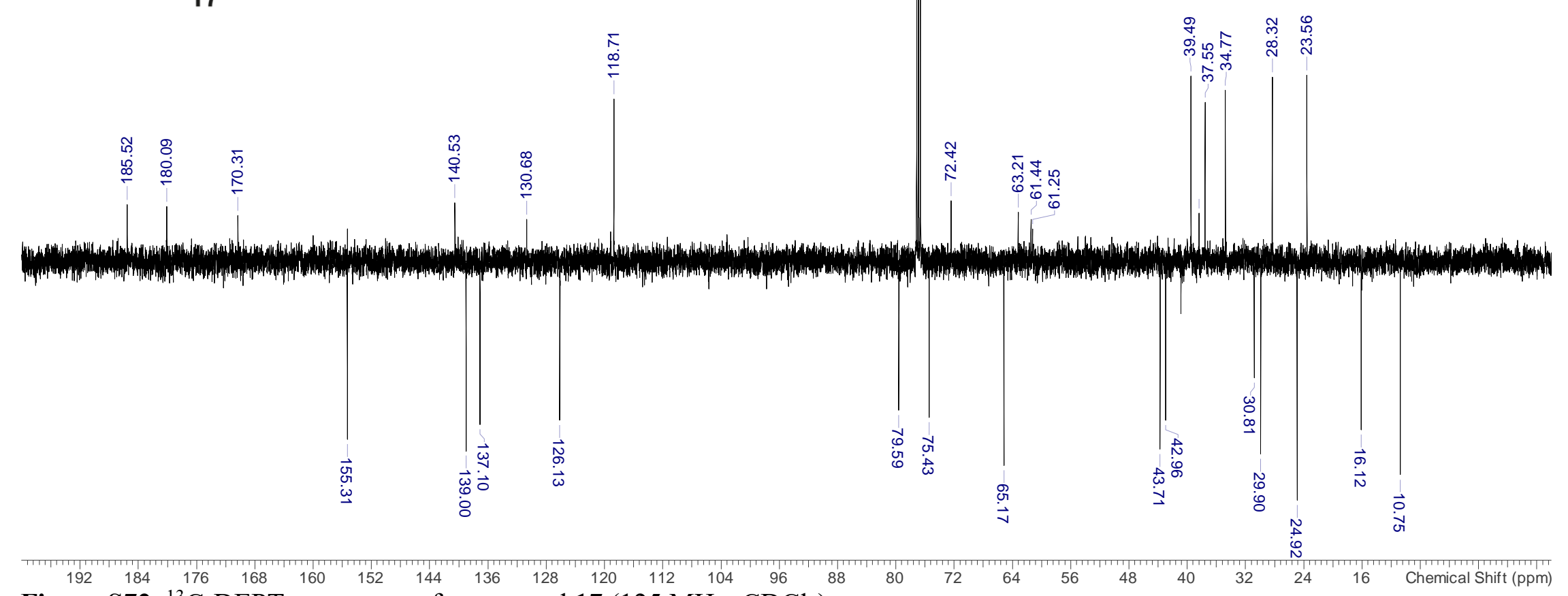

Figure S72. ${ }^{13} \mathrm{C}$-DEPTq spectrum of compound $17\left(125 \mathrm{MHz}, \mathrm{CDCl}_{3}\right)$ 


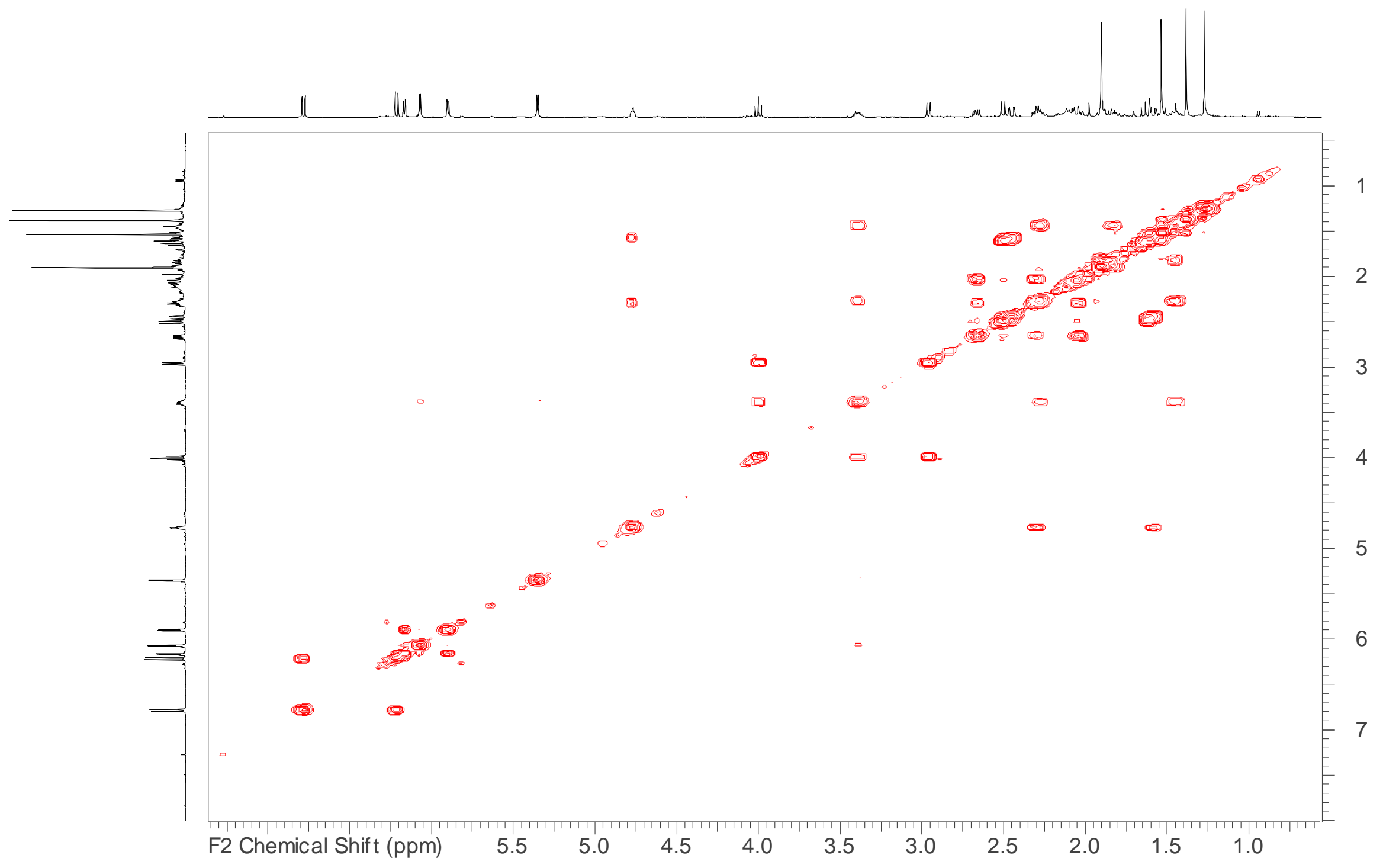

Figure S73. ${ }^{1} \mathrm{H}-{ }^{1} \mathrm{H}$ COSY spectrum of compound $17\left(500 \mathrm{MHz}, \mathrm{CDCl}_{3}\right)$. 


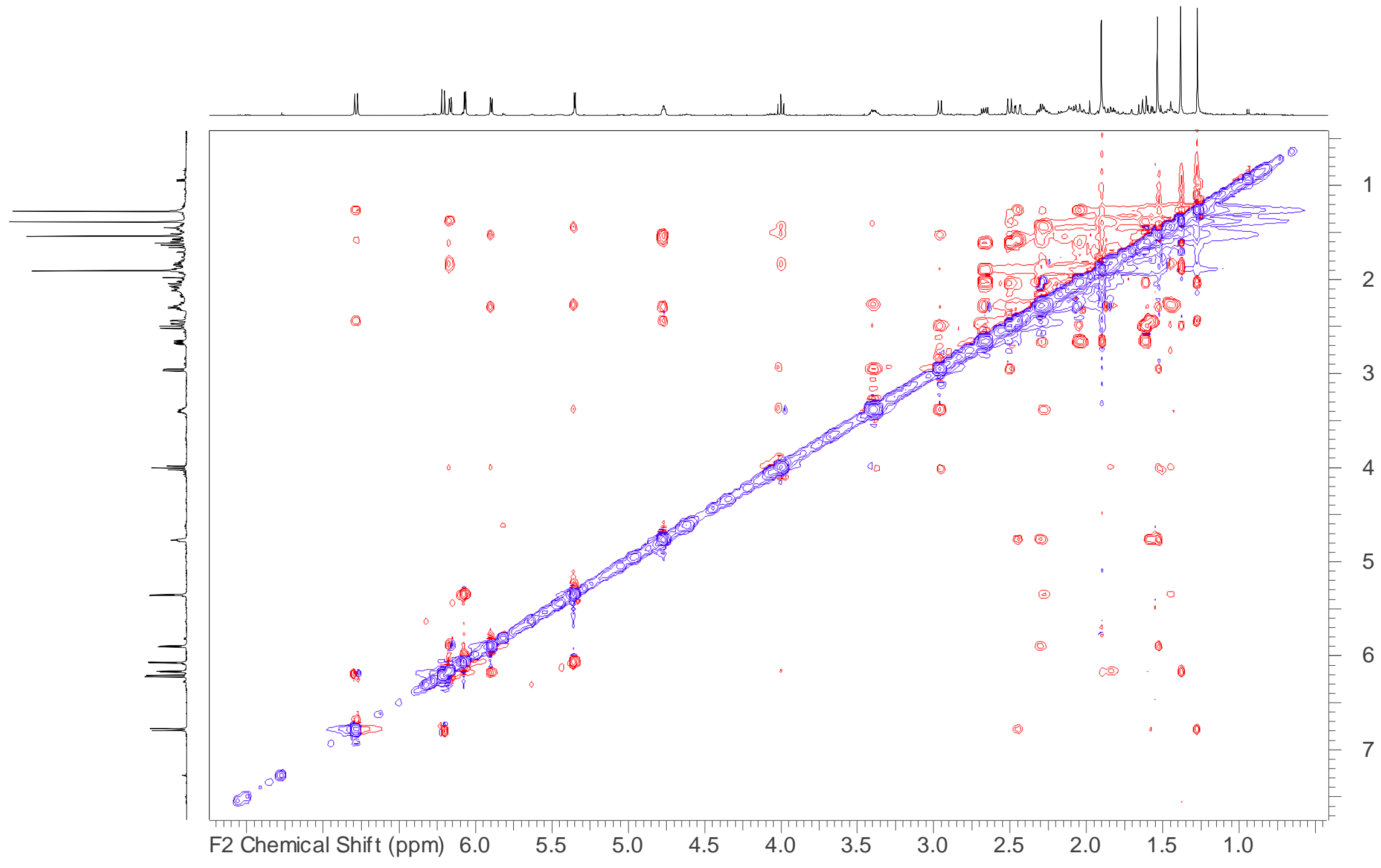

Figure S74. ${ }^{1} \mathrm{H}-{ }^{1} \mathrm{H}$ NOESY spectrum of compound $17\left(500 \mathrm{MHz}, \mathrm{CDCl}_{3}\right)$. 


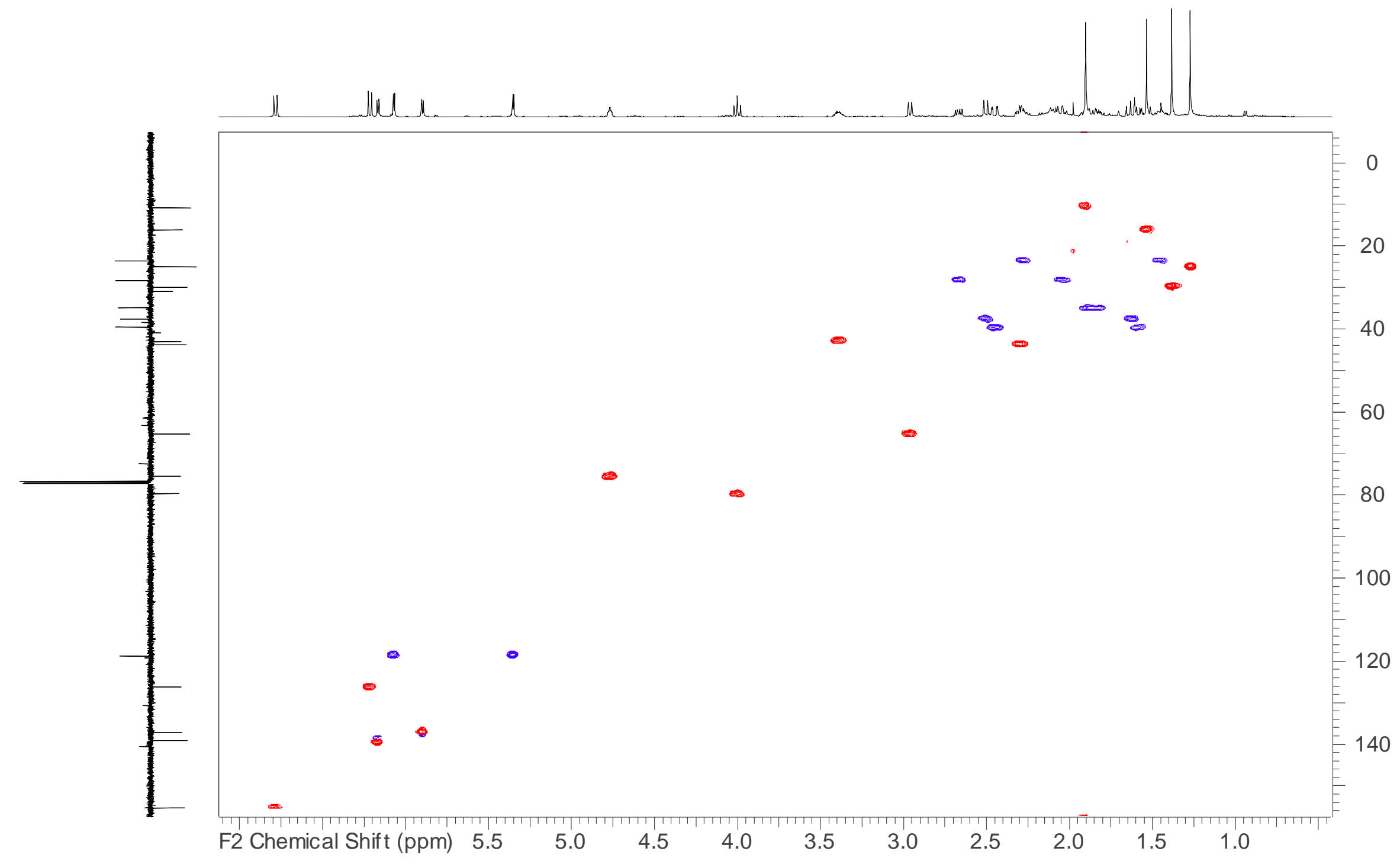

Figure S75. HSQC-DEPT spectrum of compound $17\left(500 \mathrm{MHz}, \mathrm{CDCl}_{3}\right)$. 


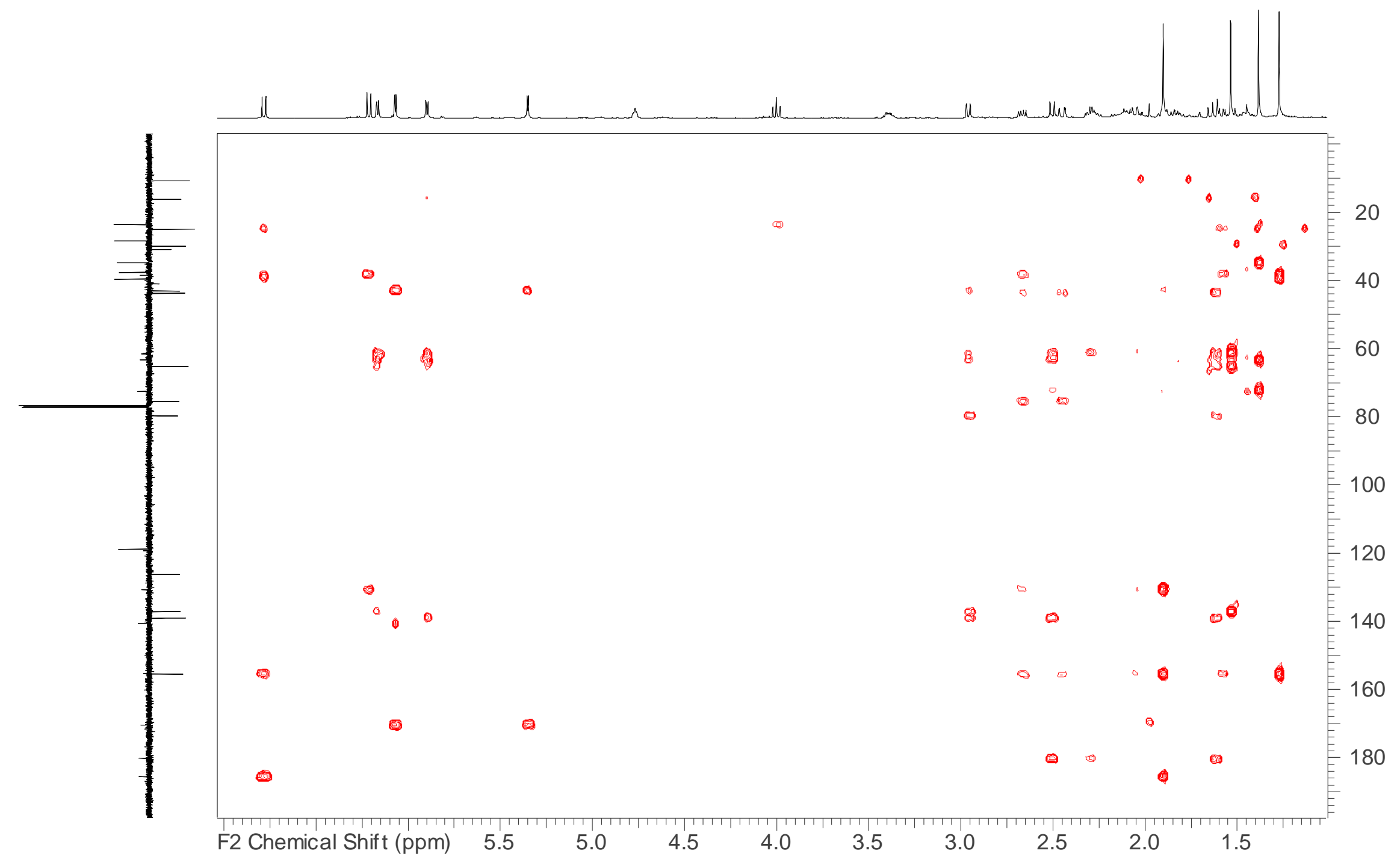

Figure S76. HMBC spectrum of compound 17 (500 MHz, $\left.\mathrm{CDCl}_{3}\right)$. 


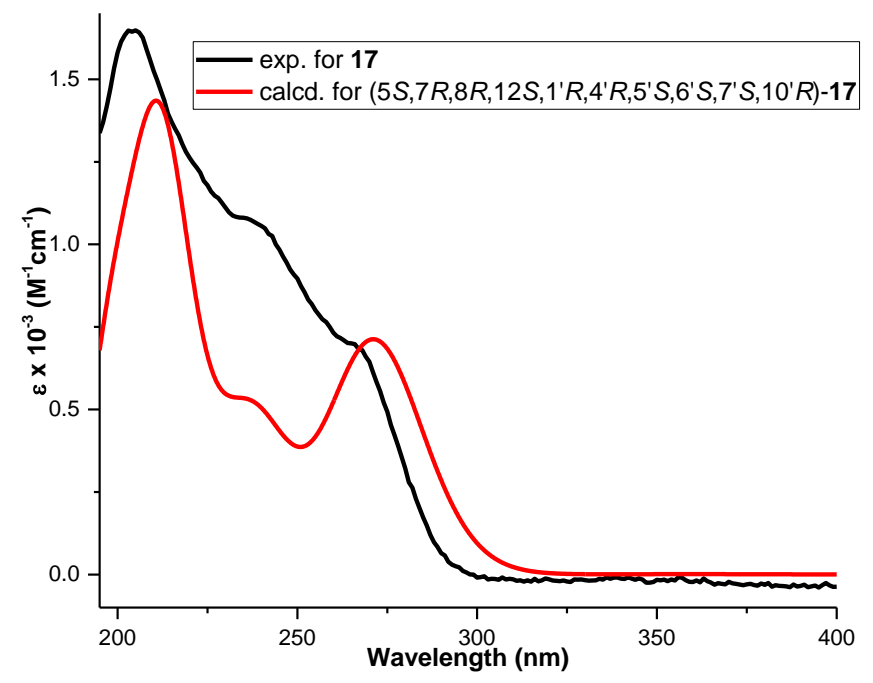

Figure S77. Comparison of experimental and computed UV spectra for compound $17 \mathrm{in} \mathrm{MeOH}$. 


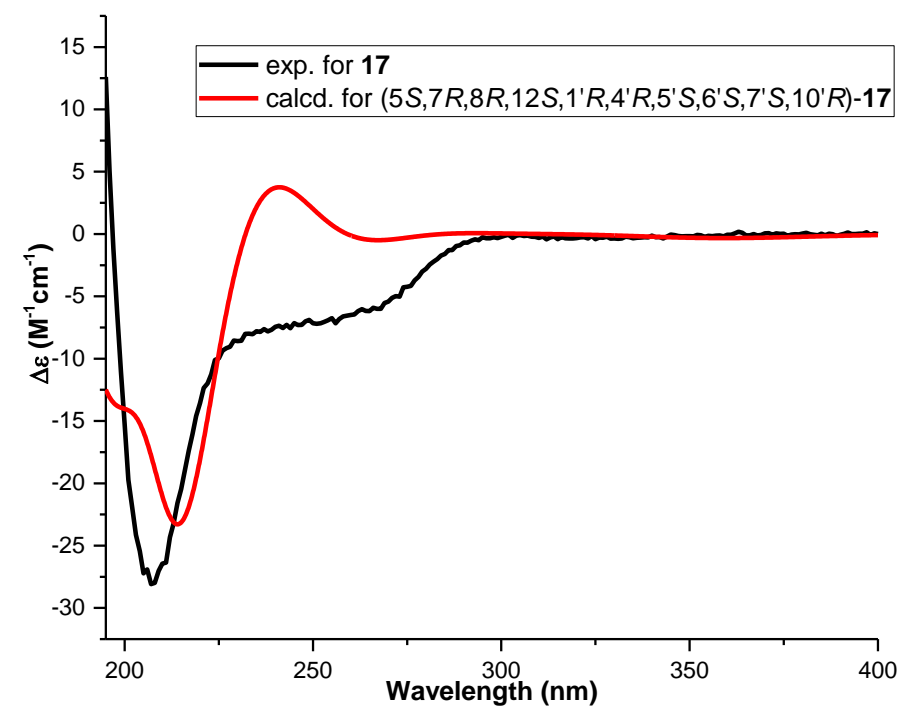

Figure S78. Comparison of experimental and computed ECD spectra for compound $\mathbf{1 7}$ in $\mathrm{MeOH}$. 


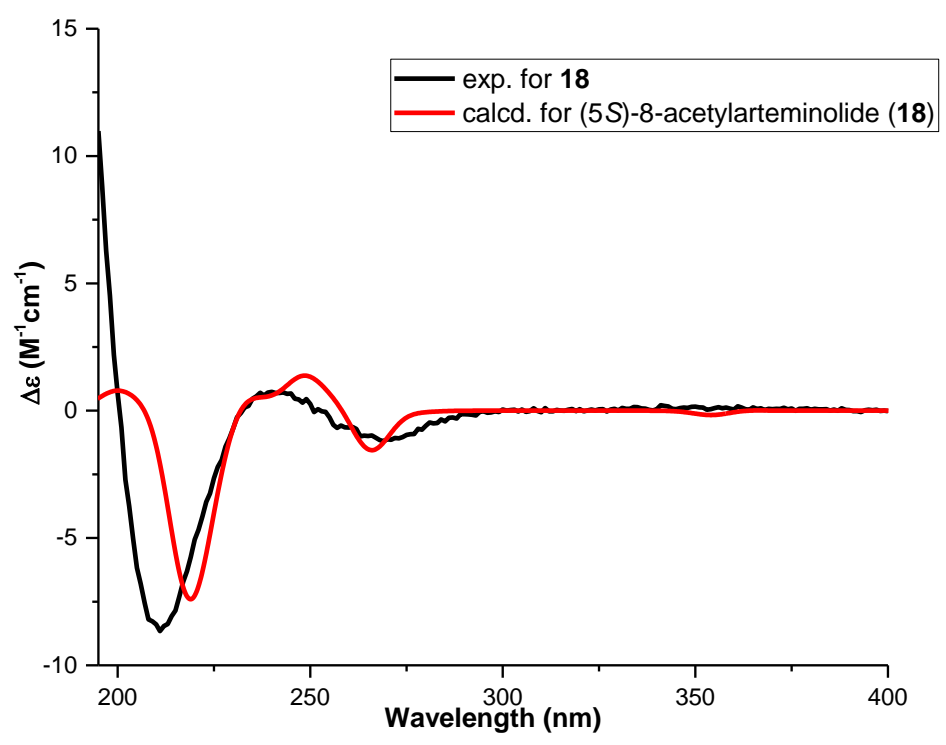

Figure S79. Comparison of experimental and computed ECD spectra for compound $\mathbf{1 8}$ in $\mathrm{MeOH}$. 


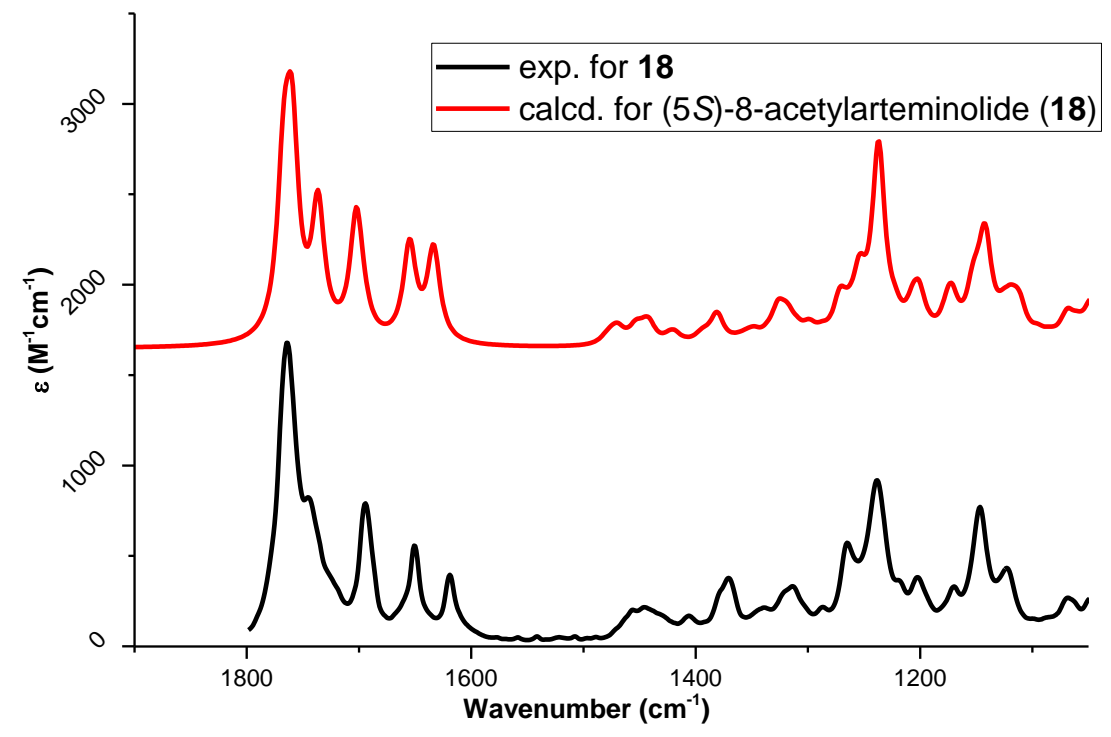

Figure S80. Comparison of experimental and computed IR spectra in chloroform for compound $\mathbf{1 8}$ in the region $19001050 \mathrm{~cm}^{-1}$. Computed spectrum of $\mathbf{1 8}$ was scaled according to the maximal SimVCD value $(0.9845)$. 

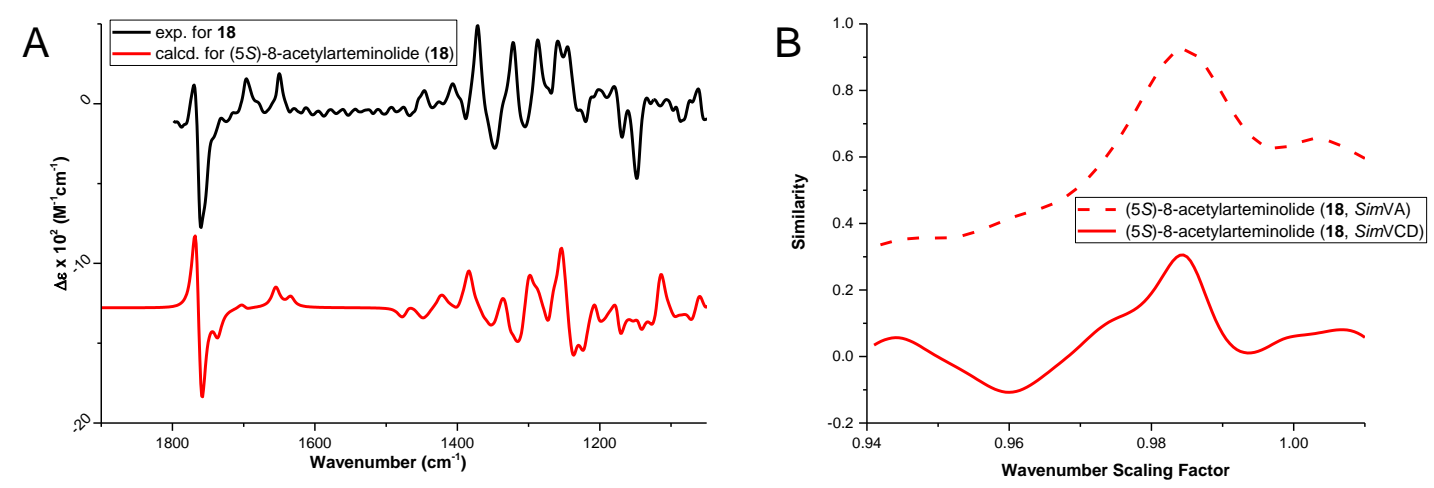

Figure S81. Comparison of experimental and computed VCD spectra in chloroform for compound 18. The region of $1900-1050 \mathrm{~cm}^{-1}$ is shown (A). Similarities ( $\operatorname{SimVA}$ and $\operatorname{Sim}$ VCD) of the experimental VA and VCD spectra of $\mathbf{1 8}$ to the calculated spectra of possible stereoisomers were plotted as functions of wavenumber scale factor (B). The wavenumber scale factor corresponding to the maximal SimVCD value in B $(0.9845)$ was used to scale the computed spectra in A. 